\title{
Explaining Interspecific Variation in Susceptibility and Resistance to Parasitism in Damselflies
}

\author{
By
}

Julia J. Mlynarek

A thesis submitted to the Faculty of Graduate and Postdoctoral Affairs in partial fulfillment of the requirements for the degree of

Doctor of Philosophy

in

Biology

Carleton University

Ottawa, Ontario

(C)2014

Julia J. Mlynarek 


\begin{abstract}
Parasites are often overlooked in evolutionary and community ecology studies. The interactions between hosts and their parasites can have important implications on community structure. Past research has focused on species-specific characteristics of parasites to explain why different, but phylogenetically closely related host species, are under different selection regimes imposed by parasites. However, the evolutionary ecology of hosts is also expected to have important influence in their associations with parasite species. This thesis explores host factors principally that are expected to influence host susceptibility and resistance, in damselflies parasitized internally by gregarines and externally by water mites.
\end{abstract}

There was often considerable interspecific variation in parasitism. When comparing species grouped into sibling species pairs, gregarine parasitism was explained in part by geographic range size of host species, but in most of the cases where there was difference in parasitism between the two closely related host species, it was the host species with the smaller range that had higher levels of parasitism. A similar pattern was observed in Arrenurus water mites parasitizing the same host species, grouped into species pairs. Additionally, Arrenurus species richness was more similar within species pairs than across species pairs meaning that more closely related hosts share similar Arrenurus fauna. At a higher taxonomic level where the host species were not grouped into species pairs, but where host phylogeny was controlled for through comparative methods, host phenology and geographic range size were better predictors of parasite host interactions than were other host characteristics such as host local abundance and host body size. The best predictor models demonstrated that host species most active in 
the middle of the damselfly flight season and with larger geographic ranges had highest levels of parasitism. There was also a positive relationship between host regional representation and the number of parasite species a host species harboured.

Interspecific variation in resistance to parasitism and levels of constitutive measures of innate immunity (PO activity) was also documented. However, the species that had highest levels of PO activity were not the ones that showed highest resistance. The most resistant hosts also tended to be the ones with the smaller geographic ranges. In a case study, the ecologically restricted host species (Nehalennia gracilis) resists all its mites in a novel way; the more widespread host species (Nehalennia irene) does not resist any individuals of the same species of parasite, determined by genetic barocding. Resistance evolution in $N$. gracilis could be because this species was able to evolve parasite recognition for a species from a closed population.

The findings of this thesis research further our understanding of host-parasite associations across phylogenetic and geographic scales. I suggest researchers need to be conscious of the complexity of the host-parasite associations and need to consider multiple host-multiple parasite systems to be norm. 


\section{Preface}

\section{Co-authorship Statement}

My contributions to the research described in this thesis were: (1) I proposed and developed the research questions in partnership with Dr. M. R. Forbes, and was primarily responsible for the design of the projects used to address these questions; (2) I was primarily responsible for carrying out all the lab work (e.g., dissections, phenoloxidase assays, mounting of larval Arrenurus) from the field work in 2010 at the Queen's University Biological Station (QUBS).

I informally supervised a summer lab assistant (A. Morrill) who helped to process damselflies collected from QUBS in 2010. The molecular work, CO1 DNA barcoding of Arrenurus were carried out by Wayne Knee, Ag. Can (Chapters 3, 4), and at the Barcode Institute University of Guelph (Chapter 6); (3) I analysed all of the data; and (4) I wrote all first drafts of the chapters/manuscripts.

I used the integrated thesis format and therefore each data chapter was formatted as an independent research article that has either been published in or was submitted to a peer-reviewed journal when this thesis was completed. There is some repetition in introductions and discussions; however I have cross-referenced between chapters to reduce repetition in the methods sections. As indicated above, I always played a major role in the design of the research and in the preparation and writing of each chapter/manuscript. However, I must acknowledge the constructive guidance and advice from my co-authors. My supervisor, Dr. M. R. Forbes contributed his theoretical, statistical and grammatical knowledge to each of my six chapter/manuscript drafts listed below. Dr. C. Hassall was a great asset in helping with the statistical analyses and 
contributed grammatical expertise to the drafts of chapters/manuscripts one. Dr. W. Knee, as mentioned before, conducted the molecular barcoding and provided feedback to the drafts of chapters/manuscripts three, four and six. Dr. A. Iserbyt was a provided guidance on the phenoloxidase assay analysis and statistical and grammatical expertise on the drafts for chapter/manuscript five. Lastly, Dr. L. Nagel contributed statistical and grammatical expertise and to the drafts of chapters/manuscripts five.

I have permission from each publisher to reproduce published manuscripts in my thesis. I have also received permission from each of my co-authors to use the collaborative work in this thesis. A specific chapter that has been published elsewhere must be cited using the journal citation information provided below. However, to reference my thesis as a whole or an unpublished chapter, I recommend using the following citation:

Mlynarek, J.J. 2014. Explaining Interspecific Variation in Susceptibility and Resistance of Parasitism in Damselflies PhD Thesis, Carleton University, Ottawa, Ontario, Canada.

\section{Chapters - Manuscript status at time of thesis completion}

1- Mlynarek, J.J., Hassall, C., and Forbes, M.R. 2012. Higher gregarine parasitism often in sibling species of host damselflies with smaller geographic distributions. Ecological Entomology 37: 419-425.

2- Mlynarek, J.J., Knee, W., and Forbes, M.R. Relative geographic range of sibling species of host damselflies does not reliably predict differential parasitism by water mites. BMC Ecology 13:50. 
3- Mlynarek, J.J., Knee, W. and Forbes, M.R. Host phenology, geographic range size and regional representation explain interspecific variation in damselfly-water mite associations. Submitted to Ecography.

5-Mlynarek, J.J., Knee, W. and M.R. Forbes, 2014. Explaining susceptibility and resistance to a multihost parasite. Evolutionary Biology 41: 115-122. 


\section{Acknowledgments}

My supervisor Dr. Mark R. Forbes provided great support and encouragement throughout my graduate career. Mark always had time to discuss of ideas and projects and always encouraged me to publish my work. I would also like to thank my committee members, Drs. Naomi Cappuccino and Jeremy Kerr. Both Naomi and Jeremy provided my valuable advice that helped me steer the progress ion of my thesis in the right direction and ask very relevant and sometimes hard questions that made the chapters of my thesis stronger.

This thesis would not have been possible without help from my collaborators (Drs. Christopher Hassall, Arne Iserbyt, Wayne Knee and Laura Nagel). The expertise of each one was intricate in the success of my research. Chris' knowledge of statistical programs and geographic distribution made my ideas and projects possible. Arne's familiarity of Phenoloxidase activity allowed me to navigate the world of insect innate immunity. Wayne's intricate understanding of the world of mites and barcoding provided expert advice and this thesis would not be what it is without him. Finally Laura's familiarity of the damselfly-water mite system at the Queen's University Biological Station made work a lot easier.

I tip my hat to all the faculty and staff at in the Biology Department. I must especially thank Dr. T. Sherratt who was always available to discuss analyses. This research would not have been possible without the space and the equipment that Drs. M. Smith, A. Golshani, J. Vierula, O. Rowland and S. Hepworth provided. Laura Thomas, M. O’Farrell, L. Chiarelli, E. Bruggink and K. Stamplecoskie were always extremely helpful. Finally J. Mallett and M. Weber made TAing a wonderful experience. 
The people in the Forbes lab (past and present) have been great. Previous students: Greg Bulte, Wayne Knee, Laura Nagel, Victoria Putinsky, Stacey Robinson and Will Shim provided comments in the first stages of my thesis. Current members: Rozlyn Butin, Sam Iverson, Andre Morrill, Jennifer Provencher, Jake Russell-Mercier and Jennifer Schellinck were helpful in discussion at the last stages of my research during the Forbes lab meetings or at the pub.

This thesis would not have been possible without input from two well established water mite taxonomists, Bruce Smith and Ian Smith. Their input helped plan my field collecting. Of course field collecting would not have been possible without the permission to collect as sites. Frank Phelan and Mark Conboy made work at QUBS a blast. I thank Nature Conservancy Quebec for allowing me to work at Large Teafield and Marlington bogs, LAMRAC for collecting at Marais de cerise, Corp. conservation du boise de Johnville for collecting at Johnville Bog, Raisin river conservation authority for collecting at Cooper's Marsh, NCC for collecting at Stony Swamp.

I need to give a big thanks to family and friends for their support and encouragement. I think all the members of Darwin's Bulldogs, most past students of the Biology department, helped me get the exercise I needed to be able to keep going. I have to thank my hiking partner and very dear friend Dr. T. Wheeler, without whom I would have been lost and stressed out. My parents (Elzbieta and Jacek Mlynarek) have always encouraged me to do the best I can in whatever I decided to do. They always happily jumped to spend time with me in the field or to look after my dog (Maya) if work needed me to work long days. Even Maya has been very supportive in her unique dog-way and made sure I got exercise, fresh air and took breaks to be able to function properly. 


\section{Table of Contents}

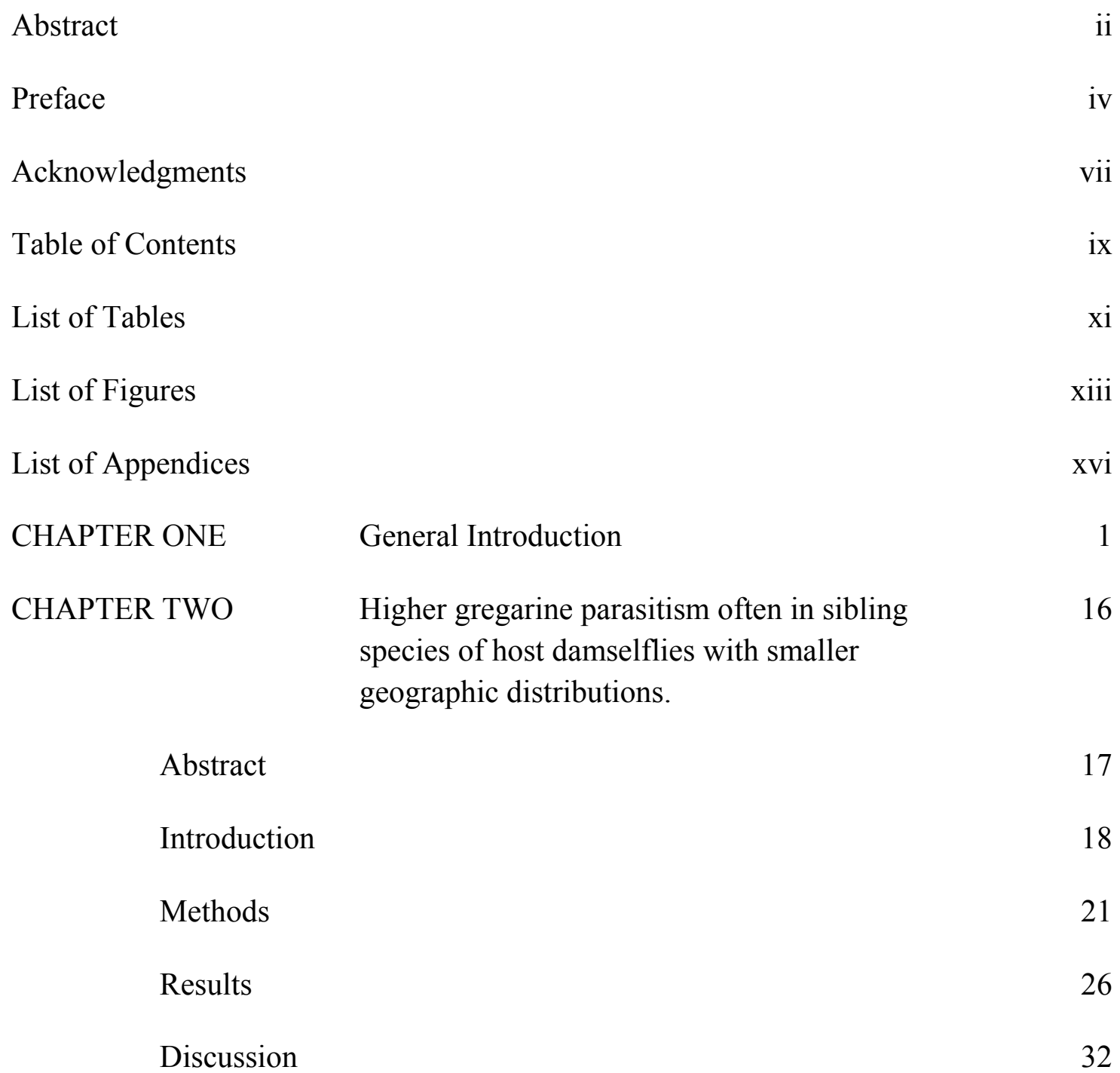

CHAPTER THREE Relative geographic range of sibling species of

host damselflies does not reliably predict differential parasitism by water mites

Abstract

Introduction

Methods

Results

Discussion 
CHAPTER FOUR

Abstract

Introduction

Methods

Results

Discussion
Host phenology, geographic range size and regional representation explain interspecific variation in damselfly-water mite associations.

Abstract
Introduction
Methods
Results
Discussion
CHAPTER FIVE
$\begin{aligned} & \text { Constitutive measures of immunity do not explain } \\ & \text { resistance across Coenagrionidae (Zygoptera)- } \\ & \text { Arrenurus (Arrenuridae: Acari) associations. }\end{aligned}$

Abstract

Introduction

Methods

Results

106

Discussion host parasite
Abstract
Introduction
Methods
Results
Discussion 


\section{List of Tables}

Table Title Page

2.1 Details of parasitism in 14 species of damselflies sampled at six sites. 27

2.2 Results of the Chi-square test for comparing prevalence and 29

Bootstrap t-test for mean intensity of parasitic infection within the seven Zygopteran species pairs.

3.1 Details of parasitism in 10 species of damselflies sampled at four sites. $\mathrm{N}$ represents the number of individuals of each species sampled.

3.2 Results of the Chi-square test for comparing prevalence and 52 Bootstrap t-test for mean intensity of parasitic infection within the five Zygopteran species pairs.

4.1 Study sites and their coordinates included in this study in 71 Southeastern Ontario and Southwestern Quebec.

4.2 Host parameters of the 16 damselfly species that were collected 80 during the reproductive season of 2011 from 13 sites in Southeastern Ontario and Southwestern

4.3 Model selection results for PGLSs evaluating water mite prevalence 85 in relation to host distribution attributes.

4.4 Model averaged regression coefficients ( $\beta$ ) for Phylogenetic 86 Generalized Least Squares (PGLSs) evaluating variation in water mite prevalence.

4.5 Model selection results for PGLSs evaluating water mite mean intensity in relation to host distribution attributes. 
4.6 Model averaged regression coefficients $(\beta)$ for Phylogenetic 88 Generalized Least Squares (PGLSs) evaluating variation in water mite intensity.

4.7 Model selection results for PGLSs evaluating water mite species richness (number of OTUs per host species) in relation to host distribution attributes.

4.8 Model averaged regression coefficients $(\beta)$ for Phylogenetic 90 Generalized Least Squares (PGLSs) evaluating variation in water mite OTUs

5.1 Sample size (N) and number of hosts that were infected by Arrenurus spp., prevalence of resisted mites and PO activity in 10 species of damselflies, grouped into five species pairs based on phylogenetic relatedness.

5.2 ANCOVA results of the differences in resistance and PO activity within each damselfly species pair.

6.1 Prevalence (\%) with 95\% confidence interval (CI) and median intensity with confidence limit and actual confidence level (CL) of mites parasitizing two focal and two transient host species in Hebert and Westport bogs 


\section{List of Figures}

Figure Title

Page

2.1 The difference in gregarine prevalence between species, within 30 species pairs.

2.2 The difference in gregarine mean intensity between species within species pairs.

3.1 Within species pair difference in measures of water mite prevalence between species.

3.2 Within species pair difference in measures of water mite mean intensity between species.

3.3 Majority rule consensus tree of 19802 trees generated by Bayesian MCMC analysis (10 million generations) of $587 \mathrm{bp}$ fragment of COI from 77 water mites, 70 ingroup and seven outgroup specimens (TL $=1048, \mathrm{CI}=0.46, \mathrm{RI}=0.86)$ posterior probability $>50 \% /$ jackknife support.

3.4 Schematic network of damselfly-water mite OTUs.

4.1 Map of study sites included in this study in Southeastern Ontario and Southwestern Quebec.

4.2 Abundance accumulation curves for the 2011 collecting season of the 16 damselfly species collected from 13 sites in Southeaster Ontario and Southeastern Quebec.

4.3 Interspecific variation in (a) prevalence and (b) intensity of 79 Arrenurus spp parasitism for the 2011 collecting season of the 16 
damselfly species collected from 13 sites in Southeaster Ontario and Southeastern Quebec.

4.4 Composite phylogeny of the Coenagrionidae found at the thirteen sites of Southeastern Ontario and Southwestern Quebec.

4.5 Majority rule consensus tree of 18002 trees generated by Bayesian MCMC analysis (10 million generations) of $683 \mathrm{bp}$ fragment of COI from 224 water mites, 217 ingroup and seven outgroup specimens $(\mathrm{TL}=1370, \mathrm{CI}=0.396, \mathrm{RI}=0.927)$.

4.6 Summary of the important predicting factors explaining Arrenurus spp. prevalence. (a) Phenology and (b) range in relation to Arrenurus spp. prevalence.

4.7 Summary of the important predicting factors explaining Arrenurus spp. mean intensity. (a) Phenology and (b) range in relation to Arrenurus spp. intensity.

4.8 Summary of the important predicting factor explaining number of OTUs in each damselfly species. Host regional representation in relation to Arrenurus OTUs.

5.1 Differences between mite resistance (a) and PO activity (b) in ten 108 coenagrionid damselfly species grouped by species pair.

5.2 Within species pair difference in measures of water mite resistance between species.

5.3 Within species pair difference in measures of PO activity between species. 
6.1 Relative local abundance differences between $N$. gracilis $(\mathrm{Ng})$ and N. irene $(\mathrm{Ni})$ at Hebert bog $(\mathrm{Hb})$ and Westport bog $(\mathrm{Wb})$ across seven weeks of collecting.

6.2 Relative local abundance differences between $N$. gracilis $(\mathrm{Ng})$ and $N$. irene $(\mathrm{Ni})$ at Hebert bog $(\mathrm{Hb})$ and Westport bog $(\mathrm{Wb})$ across seven weeks of collecting. 


\section{List of Appendices}

\section{$\begin{array}{lll}\text { Appendix Title Page } & \text { Pag }\end{array}$}

A Operational definitions for terms bolded in text, and frequently 176 used abbreviations. 


\section{CHAPTER ONE}

\section{General Introduction}




\section{General Introduction}

One of the main goals in evolutionary ecology is to understand why certain species coexist and how they interact (Pianka 1983). Host-parasite associations are useful associations to vet and test more general principles of species interactions, largely because of the strength of the association. The two members in host-parasite associations are often so closely linked that small changes in the life history or ecology of one species should have repercussions on the nature and success of the interaction (Poulin 2007). In obligate host-parasite associations, the parasite species strives to exploit the host whereas the host species tries to limit the damage caused by the parasite (Price 1980).

Recently, it has become apparent that there are clear differences in levels of parasitism (prevalence ${ }^{1}$ or intensity) between related host species (Poulin 2007). Interspecific comparisons between hosts in measures of parasitism are becoming increasingly popular (Poulin 2007, Schmid-Hempel 2011) because they enable tests of hypotheses about factors influencing host range for parasites and parasite range for hosts. Both of these ranges are critical factors to study as nature often packages itself as many parasite species attacking a given host species and as many host species being subject to attack by a given parasite species. Such studies, often limited in their taxonomic scope, have focussed on external macroparasites in mammals (Krasnov et al. 2004), blood parasites in birds (Tella et al 1999) and observations of internal gut parasites in odonates (Locklin and Vodopich 2010). However, observational research is lacking in

${ }^{1}$ See Appendix A for the operational definition of the bolded terms. 
many host-parasite systems; levels of parasitism between many evolutionarily related host species have yet to be documented.

In addition to studies explaining measures of parasitism, research on interspecific comparisons of parasite species diversity are being carried out (Poulin 2007). Parasite species richness can be a factor that influences either prevalence or intensity of parasitism where assemblages of taxa are being studied. It has been shown that as a general rule, there is an overlap in parasite species composition in closely related host species (Bordes and Morand 2009). It is not a complete overlap because there are ecological differences between host species (Bordes and Morand 2009). Unfortunately most past studies focus on one host species-one parasite species interactions (e.g. Robb and Forbes 2005). One host-one parasite associations are rare in nature (Poulin 2007). Recently, there have been studies that have started explaining the reasons for differences in helminth and arthropod parasite species richness in mammalian hosts (see Bordes and Morand 2009). There is an expected positive relationship between parasite species richness in hosts and levels of parasitism such as prevalence and intensity of overall parasitism (Poulin 2007). It is clear that our understanding is ever increasing but there are still gaps in knowing host ranges and parasite ranges in many host-parasite relationships.

Potential factors that might explain interspecific variation in parasitism include host characteristics, parasite characteristics and habitat characteristics. Host characteristics that have been studied to explain prevalence, intensity and parasite species richness include host body size (Guégan et al. 1992), phenology (Hassall et al. 2010), 
size of the geographic range (Price et al. 1988), and local abundance (Arneberg et al. 1998). Parasite characteristics include virulence, type of life cycle (direct versus complex) and body size (Poulin 2007). Habitat characteristics include size, proximity to other similar sites, geochemistry, and age (Vaughn and Taylor 2000, Mlynarek et al. 2011). Most host-parasite association studies focus on the parasite perspective because it is believed that they are the drivers of the associations due to faster reproduction times than their hosts and, as mentioned before, the fact that parasites are under higher pressure because they need hosts to survive (Poulin 2007). The host perspective is often overlooked even though they will try to limit parasite success. Hosts life history and ecological traits can influence the strength of host-parasite interactions and should be more intently studied.

Species characteristics deemed important can be measured at different scales because the strength of species' interactions might depend on different geographic, temporal and phylogenetic scales (Hefferman et al. 2014). The importance of scale has long been understood to be critical in ecological and evolutionary studies (Wiens 1989, Levin 1992), but has been frequently overlooked in empirical studies (Tack et al. 2014). There is clearly a need of measuring host species' characteristics at different scales to determine which scale influences species associations. It is expected that more phylogenetically related host species will show similar characteristics (e.g. comparable levels of parasitism, similar habitat specialisation) because of the shared evolutionary history. In the following paragraphs, I will discuss in more detail each of the host 
characteristics that are tested for their predictive ability in explaining levels of parasitism at the host population and species levels.

There are hypotheses which generally predict positive relationships between host body size and prevalence, intensity and species richness of parasites (Price et al. 1988). There is varying support for this hypothesis. In certain vertebrate host-parasite studies, host size is a predictor of parasite prevalence and intensity (Poulin and GeorgeNascimento 2007). In bats infected by streblid bat flies, host body size was positively correlated with parasite species richness infecting the hosts but not with prevalence and intensity of infection (Patterson et al. 2008). In comparison, host body size does not explain parasitism in flea species richness on rodents (Krasnov et al. 2004) or in overall parasite load in bumblebees (Durrer and Schmid-Hempel 1995). It is therefore important to determine whether host body size is a factor responsible depending on the system being studied.

Another host characteristic that could influence levels of parasitism is host phenology (Hassall et al. 2010). Phenology is an important factor in species interactions in animals that have either a seasonal life history or life cycle, such as insects (Locklin and Vodopich 2010, Forbes et al. 2012). Phenology must be very clearly defined based on the host group studied. In insects, the phenology hypothesis can be defined as the relationship between parasitism and the date of emergence of a host species or individual (Hassall et al. 2010). It is expected that the parasites will track their life history to when there is highest density of potential hosts individuals of a species; if there is a peak in the 
host density during a certain period of the year that is when the parasites will be at their highest numbers and infection will be highest (Lajeunesse and Forbes 2002). The individuals or species that are present at that time will be under highest parasite pressure (Gillespie 2010, Forbes et al. 2012). In communities however, there is an expectation that parasite species to spread themselves out temporally to avoid competition. The phenology hypothesis is a temporal measure of the differences in host-parasite associations. Since host phenology has been shown to influence host-parasite interactions, this hypothesis should be tested with additional host-parasite associations to determine the generality of the hypothesis.

Considering spatial measures, host density or local abundance can have an important influence on the prevalence and intensity of interactions (Price et al. 1988). The main prediction is that as a hosts' local abundance increases so will its levels of parasitism (Price et al. 1988). From the host species perspective, Lajeunesse and Forbes (2002), Thompson (2005) and Grant and Samways (2007) observed that less abundant or rare host species may be smaller targets of selection for parasites than their more abundant closely related host species. Additionally, Lopez (2005) determined that there was a threshold in level of parasitism in regards to host abundance in an experiment with Tribolium beetles and their mites or aggregates of mite species. At the lowest host abundance the level of parasite prevalence was significantly lower than any of the other higher host abundances and there was no difference in parasite prevalence between the higher levels of host abundance (Lopez 2005). As with phenology, local abundance seems to be a good predictor of host-parasite interactions. So testing this hypothesis with 
several host species at several sites would be the logical next step in determining its importance in host-parasite associations.

A relationship between local abundance and host geographical range has been documented in a variety of taxa: plants, zooplankton and mammals (Gaston 1990). The question then arises as to what extent host range itself can predict levels of parasitism in hosts (Morand and Krasnov 2010). The host range hypothesis can play a crucial role in explaining interspecific variation in parasitism by generalist parasites (Thompson 2005). The host range hypothesis predicts that as the range of a host species increases in size, the more likely that host species will be a under greater parasite pressure (Thompson 2005, Morand and Krasnov 2010). According to Poulin (2007) and Morand and Krasnov (2010), the size of a host species geographic range can influence levels of host-parasite associations such as prevalence, intensity and parasite species richness. When considering a group of parasites not identified to the species level, like hematozoan blood parasites infecting diurnal birds of prey, a positive relationship has been documented between the prevalence of the aggregated parasite and host geographic range (Tella et al. 1999). This hypothesis can be easily tested by using different study systems and by determining whether specific parasite species respond the same way as a group of related parasites.

Between host local abundance and host geographic range, there is an intermediate measure, host regional representation. This intermediate measure is based on the observation that a species is not found equally at all sites across its geographic range 
(Thompson 2005). A host that is able to survive in different habitats should be present at more sites across a region and its range. It is hypothesized that the number of sites a host is present within a region will affect the levels of parasitism (Durrer and Schmid-Hempel 1995). It is predicted that a host species that has high regional representation will harbour more parasites (Durrer and Schmid-Hempel 1995). Even though the predictions to this hypothesis seem obvious, few studies have determined the effect of a hosts' regional representation on levels and species richness of parasites.

All the above hypotheses assume that the parasite is successful on the host and the host does not recognize the parasite as harmful. Animals should be able recognize nonself and try to rid themselves of the invaders through the use of their immunity (SchmidHempel 2011). Because parasites need the host to complete their life cycle, it is expected that they will try and evolve the ability to counteract host defenses (Dawkins and Krebs 1979). Evaluating ecological patterns of resistance to parasitism might aid in the explaining the differences in parasitism between hosts. Studies with closely related odonate host species have documented differential levels of immunity (Kanost and Gorman 2008) and resistance to parasites (Forbes et al. 1999, Nagel et al. 2011, Mlynarek et al. 2013, Rynkiewicz et al. 2013) but the link between immunity and resistance has not been clear (Nagel et al 2011).

The mechanisms of immunity are different between vertebrates and invertebrates. Here, I focus on immunity of invertebrates. Innate immunity protects a host from invaders. Commonly, insects encapsulate foreign substances in melanin through using the 
Phenoloxidase (PO) cascade (Soderhall and Cerenius 1998). The PO cascade has been well documented (Marmaras et al. 1996, Sugumaran 2002, Kanost and Gorman 2008, Gonzalez-Santoyo et al. 2010, Moreno-Garcia et al. 2013). PO activity is considered to measure constitutive levels of immune response, i.e. measuring immune levels in the absence of an immune challenge (Wilson and Cotter 2013). I studied the extent to which PO activity can accurately predict a hosts' ability to respond immunologically, which is not well understood.

There are other proxies of innate immunity (in addition to PO activity levels) that include degree of encapsulation response to nylon inserts, concentration of hemocytes and nitric oxide levels (Wilson and Cotter 2013). These methods have demonstrated that there are costs to innate immunity in insects (Schmid-Hempel 2005, Smilanich et al. 2009, Moreno-Garcia et al. 2013). Studies on the costs of investment in immunity have determined that hosts with higher innate immune levels have reduced host fecundity and survivorship (Cotter et al. 2004, Schwarzenbach and Ward 2007, Dubovskiy et al. 2013). This trade-off between immune function and other life-history traits does not always hold. In yellow dung flies, Scathophaga stercoraria, longevity was unaffected by levels of immunity (Schwarzenbach and Ward 2007). There are unknowns about the role of innate immunity in insects but it is expected it has a strong effect on resistance and potentially on other host characteristics.

The overarching question of my thesis is to determine the importance of host ecological factors in determining interspecific variation in susceptibility and resistance to 
parasitism. This thesis focuses on documenting and explaining the patterns of hostparasite associations and interactions within the context of multiple host species interacting with multiple parasite species that have direct life cycles. To answer this question, I am using the damselfly-gregarine (internal) and damselfly-water mite (external) host-parasite systems. Even though insects, gregarines and mites are three of the most diverse group of organisms, they have received little attention in terms of evolution and ecology of host-parasite associations. The research described in this thesis is about observing and testing hypothesis using these associations, in the greater context of host-parasite associations using the host perspective.

\section{Study system - Hosts}

This thesis uses damselflies (Odonata: Zygoptera) as model host species and their internal and external parasites to address the overarching question explaining interspecific variation in susceptibility and resistance. Damselflies are good model hosts because there have been studies on their association with parasites at local scales (see Forbes and Robb 2008). This thesis broadens the scopes of past research on damselfly host-parasite associations by using larger geographical scales and higher phylogenetic resolution, and adding an element of genetic barcoding of water mites for higher taxonomic resolution. Additionally, in Eastern Canada, where this research was conducted, there is a manageable number of damselfly host species (ca. 69 species overall). Life history (e.g. development from egg to adults) across damselfly host species is similar even though there are differences in phenology (Hassall et al. 2007). Furthermore, damselflies are one of the most parasitized orders of insects; on average $20 \%$ of damselflies are infected with 
some sort of parasite (Corbet 1999). An additional, unique feature of damselflies is that resistance to external parasites can be easily recognized (Forbes et al. 1999).

\section{Study system - Parasites}

As indicated, there are two groups of parasites examined in this thesis. The internal parasites are gregarines and the external parasites are water mites of the genus Arrenurus Dugès. Both groups are species rich groups with ca. 1600 currently described gregarine species (Rueckert et al. 2011) and ca. 1000 described Arrenurus species (Smith et al. 2010).

Gregarines (Protozoa: Apicomplexa) are an interesting group that is only starting to receive recognition in ecological studies but they are one of the most successful parasites in invertebrates and arthropods. Gregarine parasites are passively-transmitted, protozoan gut parasites of most invertebrates (Bush 2001). In odonate hosts, gregarines are ingested as oocysts by the host and then migrate to the mid-gut where they develop ( $₫$ bro 1974). Once they reach sexual maturity, gregarines in the insect gut form gametocysts and are excreted by the host as oocysts (Bush 2001). The prevalence of gregarines in odonates is on average 20\%, although there is considerable variation (Corbet 1999). Abundance of infection has been recorded to vary between 1 and 900 gregarine individuals in samples of Enallagma cyathigerum Charpentier (Åbro 1974). Higher gregarine numbers were associated with lower survival, lower female egg loads, lower female mating success and shorter mate guarding in Calopteryx haemorrhoidalis Vander Linden and Enallagma praevarum (Hagen) (Córdoba-Aguilar et al. 2003, Canales-Lazcano et al. 2005). 
Arrenurus (Acari: Arrenuridae) water mites have been studied for quite a long time but not in the context of comparative ecological studies of infection from multiple host species. They are very successful on odonates but demonstrate different levels of parasitism between host species. Water mite species are considered generalists (Smith et al. 2010). Larval water mites pierce the cuticle of their host to feed. Larval mites engorge during the pre-reproductive phase of the host, (Smith et al. 2010). Prevalence and intensity of parasitic water mites varies across damselfly species. Grant and Samways (2007) detected interspecific differences in prevalence to be between $3.5 \%$ in Ceriagrion glabrum (Burmeister) and 38\% in Ischnura senegalensis (Rambur).

The difficulty with these two groups of parasites is morphological identification. Most of the existing analyses and those continued in this thesis treat each group as an aggregate, where all the species of each parasite group are considered one clade. I used molecular markers to determine Operational Taxonomic Units of water mites (i.e. barcodes) and additionally test for single 'species' of water mite using morphometric analyses.

\section{Chapters}

My thesis is divided into six data chapters separated into two lines of questioning. Chapters 2, 3 and 4 focus on examining host ecological attributes in explaining parasitism levels. In Chapters 5 and 6, I specifically examine the role of resistance and susceptibility to parasitism based on host immunity measures and their geographic range and local abundance. 
In my first data chapter (Chapter 2), I describe gregarine parasitism in 14 damselfly host species grouped into seven phylogenetically related species pairs collected near the Queen's University Biological Station (QUBS). I test the host geographic range hypothesis which states that host geographic range affects prevalence and intensity of parasites. I explore the general prediction that a host species with a larger geographic range will have a greater parasite burden. Because taxonomic resolution of these parasites is difficult and based on the assumption that gregarines are ecologically equivalent because most gregarine species seem to impact hosts similarly, I consider all the gregarine individuals as one functional unit.

Chapter 3 explores the same question as Chapter 2 but with a different parasite group. I describe Arrenurus water mite parasitism in ten damselfly host species grouped into five phylogenetically related species collected at sites around QUBS. For this Chapter, I observe ten damselfly species because two of the species pairs from Chapter 2 were not infected by Arrenurus water mites. As in Chapter 2, I once again focus on the role that host geographic range plays on prevalence and intensity of water mite parasitism. However this chapter also explores parasite species richness because taxonomic resolution of the parasite was possible with the use of molecular techniques (COI barcoding).

Chapter 4 aims to generalise the patterns seen in previous chapters at a larger spatial scale. It explores Arrenurus parasite burden and resistance at a broader geographic scale. In this study, I test the extent to which geographic range, regional representation, 
local abundance, phenology and body size of host species are good predictors of prevalence, intensity and species richness of water mite parasites. I expand on Chapter 3 because I examine host species, not grouped into species pairs, across many sites and by using parasite species diversity as an additional measure of parasitism. I test the generality of host range characteristics, local abundance and body size in explaining interspecific variation in parasitism.

In Chapter 5, I explore resistance and immunity in closely related damselflies. There has been a debate whether innate immunity positively correlates with levels of resistance. There is an expectation that higher resistance will be demonstrated in species with higher immune responsiveness. I use Phenoloxidase activity, a frequently used constitutive measure of innate immunity, to test the correlation across the same ten damselfly species as in Chapter 3. This study represents the most comprehensive study of the relations between levels of innate immunity and resistance to parasites, for insects. Because Chapters 2 and 3 focus on assessing the role of geographic range on parasitism, I observe whether there is a pattern between host geographic range, host resistance and innate immunity.

In Chapter 6, I further investigate resistance to Arrenurus in a pair of closely related damselflies (Nehalennia gracilis Morse and Nehalennia irene (Hagen)) with contrasting geographic ranges and habitat specialization. This case study arose from investigations outlined in Chapter 3 and 5. I contrast susceptibility of parasitism to levels of resistance in the two Nehalennia Selys species. I also explore the novel method of resisting the 
parasite. This chapter introduces a novel idea about the evolution of resistance based on host life histories. 


\section{CHAPTER TWO}

Higher gregarine parasitism often in sibling species of host damselflies with smaller geographic distributions.

This chapter formed the basis for the following publication and was reproduced with permission from the publisher, c 2012 John Wiley \& Sons, Inc:

Mlynarek, J.J., Hassall, C., and Forbes, M.R. 2012. Higher gregarine parasitism often in sibling species of host damselflies with smaller geographic distributions. Ecological Entomology 37: 419-425. 


\begin{abstract}
This study investigated interspecific variation in parasitism by gregarines, among sibling species of damselflies, in relation to relative size of geographical ranges of host species. Gregarines are considered generalist parasites, particularly for taxonomically related host species collected at the same sites or area. Prevalence and mean intensity of gregarine parasitism was obtained for 1,338 adult damselflies, representing 14 species (seven sibling species pairs) across three families within the suborder Zygoptera. Damselflies were collected at six local sites in Southeastern Ontario, during same periods over the season. Five of seven species pairs had significant differences in parasitism between sibling species. The less widespread host species was the more parasitized for four species pairs with significant differences in gregarine prevalence, and for one species pair with differences in mean intensity. Future studies on ecological determinants of parasitism among related species should examine robust measures of abundance of species and representation of species regionally.
\end{abstract}




\section{INTRODUCTION}

Similarity in traits between closely related species might be due to shared evolutionary history (Gaston 1990, Blomberg et al. 2003). In several host-parasite associations, parasitism demonstrates a phylogenetic signal (Poulin 2005, Poulin 2007, Munoz et al. 2007). For example, Krasnov et al. (2011) demonstrated how phylogenetic distance between two rodent species was important with respect to the similarity of parasite faunas. Clopton and Gold (1996) showed that a species of gregarine can successfully parasitize five species of domiciliary cockroach, but mentioned that it is difficult for this species to infect other species. Even though closely related species can share parasites, there is often interspecific variation in prevalence or intensity of infections (e.g. Yourth et al. 2002).

Other traits that vary between related species are the sizes of host geographic ranges and their local abundances. A positive relationship between geographic range and local abundance has often been presented (Hanski 1982, Brown 1984), but there are exceptions (Paivinen et al. 2005). Studies have also shown that the larger the host species' geographic range, the more parasite species it will harbour (Dritschilo et al. 1975, Price and Clancy 1983, Gregory 1990) and that prevalence of certain types of parasites can be correlated positively with host geographic range (Tella et al. 1999). Thus, species with larger geographic ranges are expected to have higher local abundance and also more parasite species and more parasites of a given species ( $c f$. Brown 1984, Arneberg et al. 1998). More locally abundant species are perhaps larger targets of selection for parasites than are less abundant or rare species (Lajeunesse et al. 2002, 
Grant and Samways 2011). As such, parasites are thought to be more capable of encountering and/or infecting more locally abundant species.

Another trait that varies between host species is immune response to parasites (Forbes et al. 1999, Yourth et al. 2001). In four species of damselflies parasitized by a generalist ectoparasite, Yourth et al. (2001) concluded that temporal and spatial variation in abundance did not fully explain interspecific variation in immune response. However, immune response may be expected to correlate with geographic range (Thompson 2005). As a host's geographic range increases, the more parasite species it should encounter, which leads to that host species evolving stronger generalised immune responses (Thompson 2005). This could lead to observations where the more widespread and locally abundant host species are less parasitized.

Gregarine parasites are protozoan gut parasites of invertebrates (Cielocha et al. 2011). They are ingested as oocysts by the host and then migrate to the mid-gut where they develop (Åbro 1974). On average one of five host individuals will be infected by these parasites (Corbet 1999). In a specimen of Enallagma cyathigerum (Charpentier), abundance of gregarines infection was of 900 gregarine individuals (Åbro 1974). Higher gregarine numbers have been associated with lower life history traits of hosts (CordóbaAguilar et al. 2003, Canales-Lazcano et al. 2005).

With such substantial fitness-related effects, it is important to understand how and why gregarine infections vary between species. Within populations, variation between individuals appears to be the result of phenology, with changes in gregarine parasitism 
throughout the year (Locklin and Vodopich 2010, Forbes et al. 2012). This can take the form of an increase during the season (Locklin and Vodopich, 2010) or a unimodal relationship with a peak in the middle of the season (Forbes et al. 2012), which matches a pattern seen for ectoparasitic mites in a natural insect population (Hassall et al. 2010). However, some species of damselfly show no variation in gregarine parasitism over the season (Locklin and Vodopich, 2010). Other work suggests that the nature of the habitat may influence gregarine parasitism, with populations inhabiting artificial wetlands exhibiting lower levels of infection than populations in natural wetlands (Mlynarek et al. 2011). While time of sampling and habitat are important moderator variables to control for, neither type of study shed much light on interspecific variation in parasitism among related host species at specific sites.

In this study, I examine interspecific patterns in the prevalence and intensity of gregarine parasitism in 14 species of damselflies. The damselflies species form seven species pairs from three families (Coenagrionidae: Argia Rambur, Enallagma Enallagma May, Enallagma Chromatallagma May, Ischnura Charpentier, Nehalennia Selys; Calopterygidae: Calopteryx Selys; Lestidae: Lestes Calvert). I chose host species that are phylogenetically related to first assess whether sympatric sibling species differed significantly in gregarine prevalence or mean intensity. The same gregarine species are expected to infect sibling host species in the same habitat because gregarine parasites have been shown to be generalist parasites, including gregarine parasites of odonates (Clopton 2009). For example, Cielocha et al. (2011) collected Nubenocephalus secundus 
Hays from at least seven hosts and Nubenocephalus nebraskensis Clopton from two sympatric Argia hosts.

I tested the hypothesis that the extent of the host geographic range will explain interspecific variation in parasitism when sibling species with similar evolutionary ages of association with parasites were compared. My study controlled for locality and time of collection, thereby controlling for potential seasonal variability and latitudinal and longitudinal gradients in parasitism, which do appear to exist (e.g., Locklin and Vodopich 2010).

There are two possible, but conflicting, predictions. The first prediction is that host species with the larger range have higher measures of parasitism than a sibling species with a smaller geographic range. This prediction is expected if current day geographical range is reflective of the long term coevolution of hosts with particular parasites (cf. Tella et al. 1999). The second prediction, that host species with the larger range may have lower measures of parasitism than the sibling species with the smaller geographic range. This prediction is based on the premise that species with larger ranges interact with more parasite species and have developed higher general immunity ( $c f$. Price et al. 1988).

\section{METHODS}

\section{Zygoptera collection}

Adult female and male damselflies were sampled using aerial sweep nets between May 17 and July 15, 2010 and preserved in 95\% ethanol. Species of species pairs were 
collected much closer together in time (see below). Damselflies were all processed and stored in separate vials with individual codes. Fourteen species of Zygoptera belonging to seven species pairs (Argia, Calopteryx, Enallagma Enallagma subgenus, Enallagma Chromatallagma subgenus, Ischnura, Lestes, and Nehalennia) were collected. Target species pairs within the suborder Zygoptera were chosen because they were sympatric at collecting sites and because there were reasonable differences in sizes of their geographic ranges (minimum of $100,000 \mathrm{~km}^{2}$ difference).

I differentiated between the two Enallagma species pairs because, from current phylogenetic analyses, they are in distinct subgenera (May 2002). Each of the species pairs were collected at different sites, but at sites as close as possible to each other. The Calopteryx species pair was collected on July $2^{\text {nd }}$ and $6^{\text {th }}, 2010$ at Brassett Creek $\left(45^{\circ} 01^{\prime} 09.20^{\prime \prime} \mathrm{N}, 75^{\circ} 50^{\prime} 08.77^{\prime \prime} \mathrm{W}\right)$, the Nehalennia species pair was collected at Hebert's Bog $\left(44^{\circ} 29^{\prime} 54.69^{\prime \prime} \mathrm{N}, 76^{\circ} 24^{\prime} 53.66^{\prime \prime} \mathrm{W}\right)$ on June $7^{\text {th }}$ and $30^{\text {th }}$, Enallagma Chromatallagma and Argia species pairs were collected at the edge of Lake Opinicon June $30^{\text {th }}$-July $3^{\text {rd }}$ and from July $3^{\text {rd }}-10^{\text {th }}$, respectively $\left(44^{\circ} 33^{\prime} 56.32^{\prime \prime} \mathrm{N}, 76^{\circ} 19^{\prime} 26.46^{\prime \prime} \mathrm{W}\right)$, the species of the Enallagma Enallagma clade were collected at Barb's Marsh (44³1'27.54"N, $\left.76^{\circ} 22^{\prime} 25.89^{\prime \prime} \mathrm{W}\right)$ and Jack's Marsh $\left(44^{\circ} 32^{\prime} 1.82^{\prime \prime} \mathrm{N}, 76^{\circ} 22^{\prime} 35.31^{\prime \prime} \mathrm{W}\right)$ on May $25^{\text {th }}$ and from June $7^{\text {th }}-10^{\text {th }}$, Ischnura species pair was collected at the slow stream by Osprey Marsh $\left(44^{\circ} 30^{\prime} 43.74^{\prime \prime} \mathrm{N}, 76^{\circ} 23^{\prime} 39.32^{\prime \prime} \mathrm{W}\right)$ on July $4^{\text {th }}$ and $10^{\text {th }}$, and the Lestes were collected at Yzerinac Pond $\left(44^{\circ} 32^{\prime} 12.82^{\prime \prime} N, 76^{\circ} 22^{\prime} 58.29^{\prime \prime} \mathrm{W}\right)$ on July $7^{\text {th }}$ and $12^{\text {th }}$.

I did not measure abundance of those species per se. testing for a linkage between 
geographical range and local abundance requires many replicate samples over time taken within each replicate site for each species; something beyond the logistical scope of this study. Rather, I spent my time at sites trying to collect opportunistically over short time periods (median sample size across host species was 88; IQR 68-94; Table 2.1). My sampling at single sites was intensive to obtain reliable estimates of parasitism, but was often confined to one or a few days (up to a week), barely enough to also account for seasonal variation in abundance of species within species pairs. This schedule of sampling allowed me to include more species pairs and more sites in my study, which was related to my main objective.

Although I did not measure which species was most abundant in each species pair for this study, I do note that various types of studies in my study area have been done over the years. Those studies requiring sufficient samples sizes have concentrated on the following damselfly species that are relatively abundant compared to congeners, Enallagma boreale (Hecker et al. 2002); Calopteryx maculata (Beauvois) (Forsyth and Montgomerie 1987); Nehalennia irene (Van Gossum et al. 2007); Lestes forcipatus Rambur (Yourth et al 2002); Argia moesta (Hagen) (M.R. Forbes, unpubl. study); and, Ischnura verticalis (Say) (Mlynarek et al. 2011). When this coarse index of relative abundance is considered, it appears the most abundant species based on researchers' preferences was also the most widespread species in five of six instances where this comparison could be undertaken (Table 2.1). No such studies have been done on either of the Enallagma Chromatallagma species. 
Gregarine collection

Presence or absence of gregarine infection was determined by dissecting the abdomen of each damselfly. Dissections were done by cutting the pleural membrane between the tergites and sternites and exposing the gut. The entire gut was removed, although gregarine parasitism was always restricted to the mid-gut. The gregarine trophozoites (feeding stage) were subsequently counted. The quantity of gregarine individuals within a host appears to be an important factor in determining effects on hosts (Åbro 1971). Gregarine species diversity was not considered (gregarines are treated as one "morphospecies" among species in species pairs), because there are studies that demonstrate the same species of gregarine infecting several species within a habitat. Detwiler and Janovy (2008) demonstrated that gregarine species can infect related Tribolium host species. Cielocha et al. (2011) additionally showed that the same species of gregarine, Nubenocephalus secundus infects related host species of Argia within a habitat. Based on morphometric observations, the Calopteryx species pair had distinct gregarines from the other host species. Otherwise, there was overlap in the characters in the gregarine fauna in the remaining host species; therefore, I was unable to differentiate morphospecies. I make the assumption that that gregarine species are the same between host species within species pairs (cf. Clopton, 2009).

\section{Zygoptera distribution}

All the host species used in this analysis are restricted to the Nearctic region. The size of the geographic range was assessed using the Odonata Central database (Abbott 2007). The database is actively managed and an expert verifies all records. A $2^{\circ}$ latitude- 
longitude grid was superimposed on the geographic data and the presence-absence was calculated for each cell. Once the presence was determined within a cell, the area of the species range was determined by multiplying the area of the cells by the area in $\mathrm{km}$ that each cell represents (see Hassall 2011 for details).

\section{Statistical analyses}

Hecker et al. (2002) determined that, overall, there was a significant host sex bias in gregarine parasitism levels in E. Enallagma boreale, but this bias was diminished or lost when samples from the same site and same date were compared. Furthermore, Mlynarek et al. (2011) and Forbes et al. (2012) reported no sex bias in I. verticalis or in $N$. irene respectively. In the present study, I found no sex bias differences in any species apart from E. boreale (the same species studied in Hecker et al. (2002)).

Prevalence was defined as the proportion of hosts infected with at least one gregarine parasite (Bush et al. 1997). Clopper-Pearson 95\% confidence intervals (Zar 1999) were calculated for prevalence estimates and are included. I used chi-square test to determine differences of gregarine prevalence between host species within species pairs. I ran Bootstrap 2-sample t-tests (with 2000 bootstrap replicates) to test for differences in intensity. Both the chi-square and Bootstrap t-tests were performed for each species pair separately, giving a total of 14 tests. All analyses were conducted in QP3.0 (Rózsa et al 2000).

To test for effects of geographical range, I compared parasite prevalence of the geographically restricted species against that of the geographically widespread species for 
each species pair. I first asked whether there were significant variation in either prevalence or intensity of gregarine infection between species in species pairs and then whether any such differences were explained as the more widely distributed host having the higher prevalence or intensity.

\section{RESULTS}

A total of 1,338 damselflies were collected for this study (Table 2.1). There was considerable variation between host species in gregarine parasitism. Gregarine prevalence ranged from $1.1 \%(0.03-5.8 \%$ Clopper-Pearson 95\% CI) in Argia violaceae, to $67.9 \%$ (56.7-79.8\% 95\% CI) in E. boreale (Table 2.1). Mean intensity was also variable ranging from 1 (N/A 95\% CI) in Argia moesta to $24.8(17.6-34.195 \%$ CI) in Calopteryx aequabilis.

From the chi-square tests, four of the seven species pairs (Calopteryx, Enallagma Chromatallagma subgenus, Ischnura and Nehalennia) showed significant differences in prevalence between the species in the species pairs. Based on the Bootstrap 2-sample t-test, one of seven species pairs (Calopteryx) also had statistically significant different mean intensities between the two species. Thus in four of the seven species pairs, there were significant or near significant differences between species members in one or more measures of parasitism. 
Table 2.1. Details of parasitism in 14 species of damselflies sampled at 6 sites. N represents the number of individuals of each species sampled. Geographic range of the host is provided. Relative abundance represents the species of a species pair that has received more attention from researchers in my study area, because of the need for large samples. Confidence limits for prevalence and median intensity are shown respectively. $\mathrm{RA}=$ Relative abundance $\mathrm{LO}=$ Lake Opinicon, $\mathrm{BC}=$ Brasset Creek, JM $=$ Jack Marsh, $\mathrm{OM}=$ Osprey Marsh, YP = Yzerinac Pond, $\mathrm{HB}=$ Hebert Bog.

\begin{tabular}{|c|c|c|c|c|c|c|}
\hline Host species & Location & $\mathrm{N}$ & $\begin{array}{c}\text { Geograph } \\
\text { range } \\
\left(10^{6} \mathrm{Km}^{2}\right.\end{array}$ & & $\begin{array}{c}\text { Prevalence \% } \\
\quad(95 \% \mathrm{CI})\end{array}$ & $\begin{array}{c}\text { Mean } \\
\text { Intensity } \\
(95 \% \mathrm{CI})\end{array}$ \\
\hline Argia moesta & $\mathrm{LO}$ & 88 & 4.10 & $>$ & $\begin{array}{c}1.1 \\
(0.03-6.2)\end{array}$ & $\begin{array}{c}1 \\
(\mathrm{n} / \mathrm{a})\end{array}$ \\
\hline Argia violacea & $\mathrm{LO}$ & 94 & 3.98 & & $\begin{array}{c}1.1 \\
(0.03-5.8)\end{array}$ & $\begin{array}{c}8 \\
(\mathrm{n} / \mathrm{a})\end{array}$ \\
\hline Calopteryx aequabilis & $\mathrm{BC}$ & 62 & 3.55 & & $\begin{array}{c}54.8 \\
(41.7-67.5)\end{array}$ & $\begin{array}{c}24.8 \\
(17.6-34.1)\end{array}$ \\
\hline Calopteryx maculata & $\mathrm{BC}$ & 68 & 3.77 & $>$ & $\begin{array}{c}25.0 \\
(15.3-37.0)\end{array}$ & $\begin{array}{c}5.2 \\
(2.1-13.9)\end{array}$ \\
\hline Enallagma $(E)$ boreale & $\mathrm{JM}$ & 68 & 6.53 & $>$ & $\begin{array}{c}69.1 \\
(56.7-79.8)\end{array}$ & $\begin{array}{c}10.9 \\
(8.1-14.1)\end{array}$ \\
\hline Enallagma $(E)$ ebrium & $\mathrm{JM}$ & 28 & 4.35 & & $\begin{array}{c}67.9 \\
(47.6-84.1)\end{array}$ & $\begin{array}{c}10.5 \\
(4.7-12.3)\end{array}$ \\
\hline Enallagma $(C)$ signatum & $\mathrm{LO}$ & 120 & 3.23 & & $\begin{array}{c}5.0 \\
(1.9-10.6)\end{array}$ & $\begin{array}{c}3.8 \\
(1-6.7)\end{array}$ \\
\hline Enallagma $(C)$ vesperum & $\mathrm{LO}$ & 98 & 2.6 & & $\begin{array}{c}16.3 \\
(9.6-25.2)\end{array}$ & $\begin{array}{c}2.5 \\
(1.5-4.2)\end{array}$ \\
\hline Ischnura posita & $\mathrm{OM}$ & 75 & 3.13 & & $\begin{array}{c}46.7 \\
(35.1-58.6)\end{array}$ & $\begin{array}{c}11.5 \\
(7.7-19.9)\end{array}$ \\
\hline Ischnura verticalis & $\mathrm{OM}$ & 23 & 4.36 & $>$ & $\begin{array}{c}21.7 \\
(7.5-43.7)\end{array}$ & $\begin{array}{c}8 \\
(1.2-16.2)\end{array}$ \\
\hline Lestes forcipatus & YP & 182 & 3.19 & $>$ & $\begin{array}{c}25.8 \\
(19.6-32.8)\end{array}$ & $\begin{array}{c}5.9 \\
(3.6-11.9)\end{array}$ \\
\hline
\end{tabular}


Chapter 2

Table 2.1. Continued

\begin{tabular}{|c|c|c|c|c|c|}
\hline Host species & Location & $\mathrm{N}$ & $\begin{array}{l}\text { GeographicRA } \\
\text { range } \\
\left(10^{6} \mathrm{Km}^{2}\right)\end{array}$ & $\begin{array}{c}\text { Prevalence \% } \\
(95 \% \mathrm{CI})\end{array}$ & $\begin{array}{c}\text { Mean } \\
\text { Intensity } \\
(95 \% \mathrm{CI})\end{array}$ \\
\hline Lestes congener & YP & 73 & 5.99 & $\begin{array}{c}17.8 \\
(9.8-28.5)\end{array}$ & $\begin{array}{c}1.6 \\
(1.2-1.8)\end{array}$ \\
\hline Nehalennia gracilis & HB & 88 & 1.51 & $\begin{array}{c}40.9 \\
(30.5-51.9)\end{array}$ & $\begin{array}{c}9.7 \\
(6.1-17.4)\end{array}$ \\
\hline Nehalennia irene & HB & 205 & 4.03 & $\begin{array}{c}55.1 \\
(48.0-62.1)\end{array}$ & $\begin{array}{c}14.6 \\
(9.5-30.2)\end{array}$ \\
\hline
\end{tabular}

When I plotted differences in prevalence between species within species pairs, I found no evidence that species with the larger geographical range had higher prevalence (Fig. 2.1). In fact, for three of the species pairs with significant differences in gregarine prevalence (Calopteryx, Enallagma Chromatallagma and Ischnura) it was the less widespread host species that was most often parasitized (see parameter estimates in Table 2.2). In contrast, in Nehalennia, the more widespread species had higher parasite prevalence. The remaining three species pairs did not show significant differences in prevalence between their sibling species, despite the fact that one of the species pairs (Enallagma Enallagma) had quite disparate geographical ranges. A similar pattern was seen in the intensity of infection, with two species pairs (Calopteryx) showing significantly lower intensity of infection in the more widespread species (Table 2.2). 
Table 2.2. Results of the Chi-square test for comparing prevalence and Bootstrap t-test for mean intensity of parasitic infection within the seven Zygoptera species pairs.

\begin{tabular}{lccccc}
\hline Host species pair & \multicolumn{3}{c}{ Prevalence } & \multicolumn{2}{c}{ Intensity } \\
& $\chi^{2}$ & $\mathrm{df}$ & $\mathrm{p}$ & t-value & Bootstrap p \\
\hline Argia & 1.12 & 1 & 0.29 & $\mathrm{NA}$ & $\mathrm{NA}$ \\
Calopteryx & 12.11 & 1 & $\mathbf{0 . 0 0 1}$ & 3.83 & $<\mathbf{0 . 0 0 1}$ \\
Enallagma $E$ & 0.02 & 1 & 0.90 & 1.07 & 0.28 \\
Enallagma $C$ & 7.63 & 1 & $\mathbf{0 . 0 0 6}$ & 0.46 & 0.62 \\
Ischnura & 6.20 & 1 & $\mathbf{0 . 0 1}$ & 0.68 & 0.50 \\
Lestes & 1.86 & 1 & 0.17 & 2.28 & 0.19 \\
Nehalennia & 4.98 & 1 & $\mathbf{0 . 0 3}$ & 1.02 & 0.31 \\
\hline
\end{tabular}




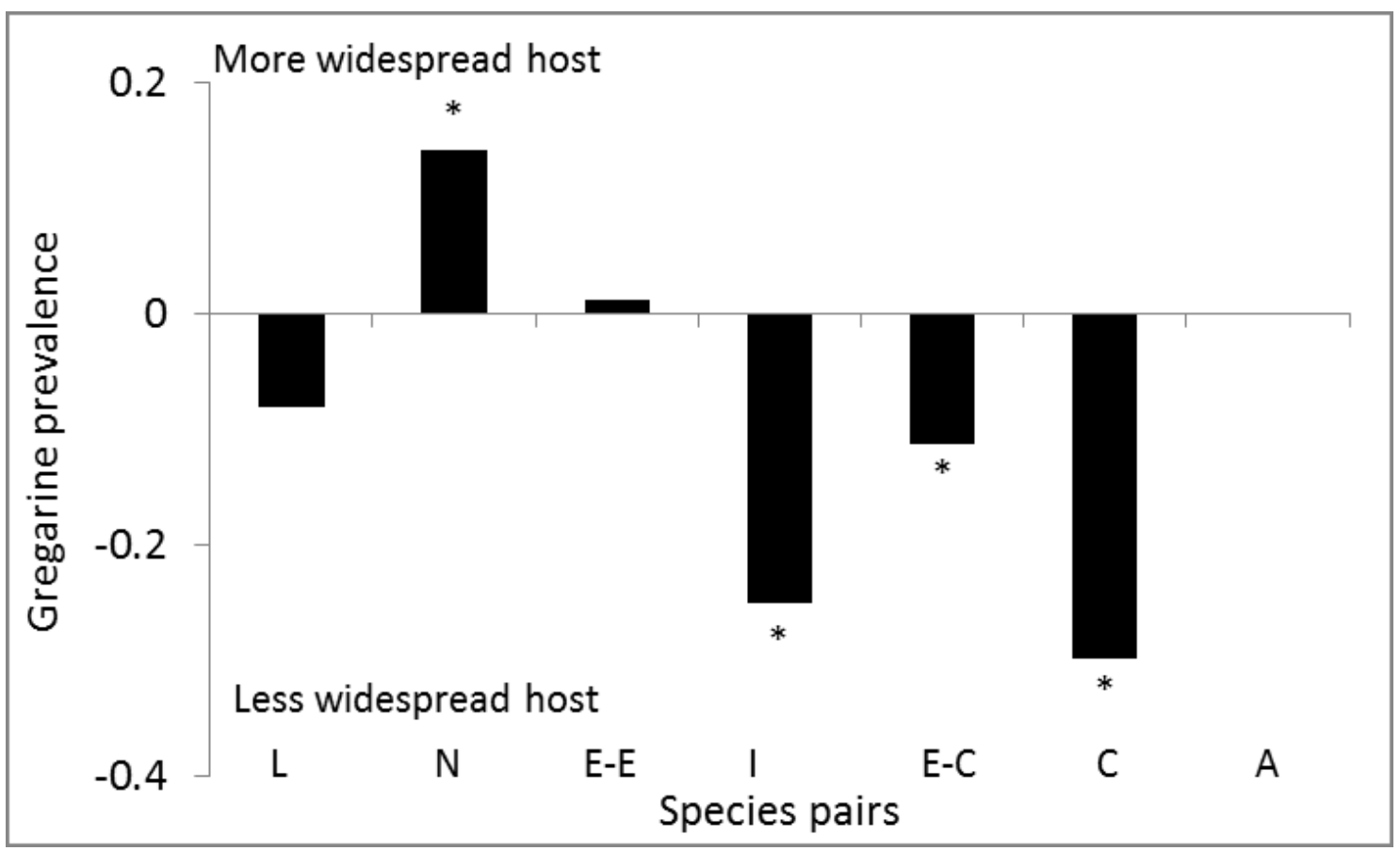

Figure 2.1. The difference in prevalence between species, within species pairs. If the difference is positive, then the more widespread species of the species pair has higher prevalence (from Table 2.1); if the difference is negative, then the species with the higher prevalence is the less widespread host. Significant differences in prevalence values are marked by * and can be seen in Table 2.2. Note that the Ischnura species pair has nearly significant difference in prevalence. Species pairs are organized from left to right as most different in geographical range to least different. Key: A=Argia, $\mathrm{C}=$ Calopteryx, $\mathrm{E}-\mathrm{C}=$ Enallagma Chromatallagma, E-E = Enallagma Enallagma, $\mathrm{I}=$ Ischnura, $\mathrm{N}=$ Nehalennia, $\mathrm{L}=$ Lestes. 


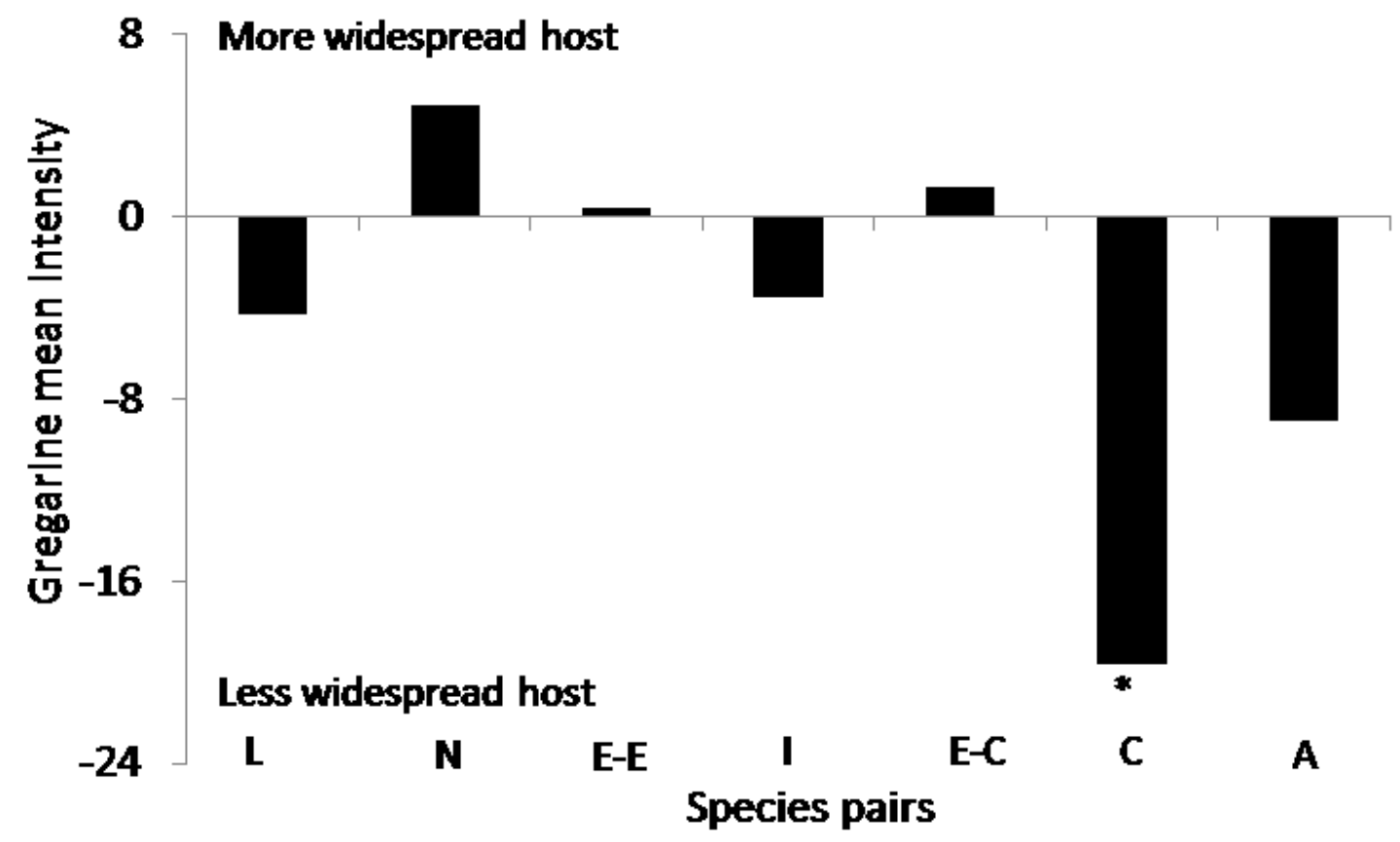

Figure 2.2. The difference in mean intensity between species within species pairs. If the difference is positive, then the more widespread species of the species pair has higher intensity (from Table 2.1); if the difference is negative, then the species with the higher intensity is the less widespread host. Significant differences in intensity values are marked by * and can be seen in Table 2.2. Note that the Ischnura species pair has nearly significant difference in prevalence. Species pairs are organized from left to right as most different in geographical range to least different. Key as in Figure 2.1 


\section{DISCUSSION}

My study was based on 14 associations between parasites thought to be generalists (gregarines), and sibling species of their damselfly hosts. I found that in several species pairs, one species often had a higher measure(s) of parasitism; however, most often it was the less widely distributed host species of a species pair. This pattern certainly occurred for Calopteryx, Enallagma Chromatallagma and Ischnura species. No pattern was evident for Argia species and the opposite pattern was present only for the Nehalennia species pair, similar to what was observed for Calopteryx species in Finland (Ilvonen et al. 2011).

Widespread species are often more abundant than less widespread species (Hanski 1982, Brown 1984). Intuitively, more numerous host species should be larger targets of selection on parasites; therefore, it is expected that parasites should be more prevalent or numerous in widespread hosts (Morand et al. 2010). Stated another way, the parasite is expected to be better adapted to the widespread and more abundant host (Price et al. 1988, Forbes et al. 1999, Ilvonen et al. 2011). There is also the possibility that widespread hosts bring their parasites with them into regions occupied by less widespread species (Durrer and Schmid-Hempel 1995). Such secondary contact could mean that one species of the species pair had associations with the parasite for a shorter period of evolutionary time.

In most cases in this study, the widely distributed host species was thought to be more locally abundant based on previous work, although this was not known a priori for 
Enallagma Chromatallagma. However for Nehalennia, the host species that was expected to be more locally abundant had higher parasitism (Van Gossum et al. 2007). These results leave two important issues unresolved. The first is why some species in species pairs show differences in parasitism, whereas others do not; and, second, why Nehalennia species appear to be an exception to the rule of less widely dispersed species having higher parasitism, when such species differences in parasitism exist. Nehalennia irene is a cosmopolitan species while $N$. gracilis is a specialist of bog habitats (Walker 1953). The remaining species pairs have similar habitat affinities and therefore are present within the same parasite communities.

The first issue identified above is perhaps the most perplexing. That is, why do species in some species pairs show differences in measures of parasitism, whereas this is not universally true? Although sibling species share age of associations with their generalist parasites, different host species pairs might have diverged for different periods of evolutionary time. Such differences might allow there to be time for differences in degree of parasitism to evolve in some species pairs, but not others. In other words, species within Enallagma and Argia pairs might have had less time for divergence from one another. However, such explanations cannot explain why the less widespread species often tend to be more parasitized (but see Ilvonen et al. 2011) or why Nehalennia species are an exception in this study.

In the Nehalennia species pair, the more widespread and more locally abundant species is under higher parasite pressure. In fact, this is also seen for ectoparasitic mites 
on these two host species, but here the more widespread host species ( $N$. irene) is susceptible to a mite species to which the less abundant, and less widespread, $N$. gracilis is resistant (Mlynarek et al. 2014). As mentioned, these patterns of parasitism do not occur for species in other species pairs. The Nehalennia species pair is very different from the other species pairs, because $N$. gracilis is highly specialised and restricted to bogs. In contrast to Nehalennia, the sibling species of Calopteryx, Enallagma Chromatallagma and Ischnura are ecologically similar within the species pairs; the Calopteryx species have evolved at creek edges, Enallagma Chromatallagma are lake damselflies and Ischnura species are usually found around ponds, marshes and slow moving streams (Walker 1953). If there was a species pair where secondary contact was likely, it might well be Nehalennia.

I note that an additional analysis should be done to see if difference in range between species in species pairs related to the magnitude of difference in measures of parasitism. While possible, such analyses are not practical; there is a low sample size of only seven comparisons and an analysis of this type will compare larger species like Calopteryx, which have morphologically distinct gregarines, with smaller species like Nehalennia. Other factors, such as whether certain gregarine parasites exist at higher prevalence on average than other gregarine species, will determine the magnitude of difference I might expect for comparisons within host species pairs. I already know from my analysis that a general pattern of increasing difference in parasitism with increasing difference in geographical range is not evident. Species pairs in Fig. 2.1 were arranged 
along a gradient of decreasing difference in geographical range and no gradient in differences in prevalence was evident.

If the parasites are not generalists, then my results still leave open the question of why some associations should be marked by higher measures of parasitism and why the balance seems to be that less widespread hosts (which also are expected to be less locally abundant on average) have higher measures of parasitism. It is possible that my less widespread species were actually more regionally widespread; however, when I chose the target host species I determined this not to be the case (Walker 1953). Another possible explanation would be that the more geographically restricted host species has not developed strong innate defences because of possible low encounter rates with parasites generally. It is believed that larger geographic ranges lead to increased levels of interactions (Pielou 1974), which might mean higher innate immune defences. This possibility can be resolved with ecological immunology studies.

In summary, I am suggesting that two factors might both influence the degree to which related host species are parasitized. The first is relative investment in innate immune defences, which might be lower on average for less widespread species: a proposition, which could be tested. The second is time in association with the host whereby parasites might evolve to be more effective generalists. If a less widespread host species has had a recent contact, it might show lowered measures of parasitism even if it is not particularly well defended, because the parasite has not yet evolved to exploit that species effectively. Less widespread species with longer contact times with parasites are 
expected to show higher levels of parasitism than their widely distributed sibling species. Studies comparing exposure of sibling species to infective stage of parasites would also prove useful in testing these ideas. 


\section{CHAPTER THREE}

\section{Relative geographic range of sibling species of host damselflies does not reliably predict differential parasitism by water mites}

This chapter formed the basis for the following publication:

Mlynarek, J.J., Knee, W., and Forbes, M.R. 2014. Relative geographic range of sibling species of host damselflies does not reliably predict differential parasitism by water mites. BMC Ecology 13: 50. 


\section{ABSTRACT}

One of the main challenges in ecological and evolutionary parasitology is to determine the factors that explain variation among host species in parasitism. In this study, I addressed whether host phylogeny or ecology was important in determining host species use by water mites. Parasitism (prevalence and intensity) by Arrenurus water mites was examined in relation to geographic range of host damselflies from sibling species pairs. In addition, the likelihood of putative mite species parasitizing both species of a host species pair was explored as was whether particular water mite species were restricted to particular habitats. A total of 1162 damselflies were examined for water mites across four sites in Southeastern Ontario. These damselflies represent ten species (five closely related host species pairs) in the Coenagrionidae. Only two of the five species pairs showed near significant or significant differences in prevalence of infection by mites. In one of those species comparisons, it was the less widespread host that had higher water mite prevalence and in the other species comparison, the less widespread host species had lower water mite prevalence. Only one of the five pairs showed a significant difference in intensity of infection; intensity was higher in the species with a smaller geographic range. Based on the COI barcode, there were nine water mite clades (OTUs) infecting these ten host species. Three Arrenurus OTUs may be host monospecific, four OTUs were specific to a given host species pair, and two OTUs infected at least three host species. Host species in each species pairs tend to share at least one of the Arrenurus OTU. No striking differences in mite species diversity were found among species in any species pair. Finally, the Arrenurus examined in this study appear to be ecological specialists, 
restricted to a particular type of habitat, parasitizing few to many of the host species present in that site or habitat. Although differences in levels of parasitism by water mites exist for some closely related hosts species, no such species differences in parasitism were found between other closely related host species. Differences in geographic range of related host species does not reliably explain differential levels of parasitism by water mites. 


\section{INTRODUCTION}

A major challenge of the combined fields of ecological and evolutionary parasitology is to understand the determinants of variation in parasitism between closely related host species (Poulin 2007, Bordes et al. 2010). Some of the proposed determinants include

size of a host species' geographic range (Dritschilo et al. 1975), host body size (Simková et al. 2001), taxonomic relatedness of host species (Krasnov et al. 2006) and habitat requirements of both hosts and parasites (Kennedy and Bush 1994, Krasnov et al. 1997). Most of the research in this area has been done on vertebrate hosts (e.g. Morand and Krasnov 2010). A question remains as to whether it is possible to extrapolate findings of studies on vertebrates and their parasites to similar problems with invertebrate host species and their parasites.

Some generalities can be drawn from studies on parasitism of vertebrates as a guide to explorations of parasitism of invertebrate hosts. For example, host species with a greater geographic range may come into contact with infective stages of more parasite species and therefore have higher diversity of parasites or higher parasitism levels (Tella et al. 1999, Poulin and Morand 2004). Alternatively, hosts with larger geographic areas might evolve more generalized immune responses and therefore have fewer parasites in local areas (cf. Mlynarek et al. 2012). Using damselflies and gregarines, Mlynarek et al. (2012) demonstrated that in four out of seven species pairs, one host species had a statistically higher prevalence of gregarines, when controlling for collecting site. In three of those species comparisons, the sibling species with the smaller geographic range had 
higher prevalence; in the latter comparison, the species with the larger geographic range had the higher prevalence. Similarly, in one out of seven species pairs, the species that had a smaller geographic range had a statistically higher gregarine intensity (Mlynarek et al. 2012).

Other studies with vertebrates and their parasites have demonstrated that host geographic range size can be related to parasite species richness (Dritschilo et al 1975, Gregory 1990, Simková et al. 2001). The recurrent relation between host geographical range and parasite species diversity has been explained in part by increased chance of intense localized encounters between interacting species with the increasing range size of the host species (Price and Clancy 1983, Poulin and Morand 2004). Although host species with larger geographic ranges are expected to have more parasite species than closely related host species with smaller geographic ranges (Poulin and Morand 2004), it is unknown whether such patterns are observed often at given sites. Species with larger geographical ranges typically occupy more sites and might be subjected to more studies: a potential problem recognized early (Gregory 1990).

Several studies have looked at relationships between host and/or habitat characteristics and parasitism levels in insect host-invertebrate parasite associations. Durrer and Schmid-Hempel (1995) studied regional and local abundance of bumblebees with respect to external and internal parasite load and diversity. They found that there was a positive correlation with parasite load and colony size, and also that parasite diversity correlated positively with regional distribution of hosts, but host body size did 
not affect any of the measures (Durrer and Schmid-Hempel 1995). Jaenike and Perlman (2002) reviewed the role of nematode parasites on mycophagous Drosophila behaviour, reproduction and community structure. Their work demonstrated that parasitism facilitates co-existence between closely related hosts and reduces fertility in female Drosophila. One other study compares parasitism levels explicitly between species differing in geographical representation, but there the focus is on gregarine parasites of calopterygid damselflies in allopatric versus sympatric associations (Ilvonen et al. 2011).

For various reasons, the extent to which parasitism and parasite diversity varies as a function of geographic range can be explored effectively using coenagrionid damselflies and Arrenurus water mites. First, there is interspecific variation in ecological and evolutionary traits in closely related damselfly host species (e.g., host geographical range, local abundance; see Mlynarek et al. 2012). Second, it is known that there are several generalist species among mites (Forbes and Robb 2008). Third, Arrenurus infection has been demonstrated to vary between closely related lestid host species in different habitats (Nagel et al. 2011) and between more distantly related host species in the same habitat (Grant and Samways 2007). Fourth, Arrenurus water mites first parasitize teneral adults and are easily recognized as unengorged but live, unengorged and resisted, and engorged external parasites. Importantly, these mites remain on one host for the duration of their parasitic life stage (Smith et al. 2010). Larval Arrenurus embed their mouthparts into the teneral cuticle of their host and secrete a stylostome or feeding tube. Mites remain usually on the ventral side of the thorax, between the legs, or the last abdominal segments (Smith et al. 2010). Once the host returns to a water body to mate 
and/or lay eggs, the water mite detaches and falls into the water to continue its life cycle. Different species of water mite are also known to inhabit different types of water bodies (Smith et al. 2010), setting the stage for particular associations to be habitat specific.

The objectives of this study were several-fold. First, I tested whether closelyrelated host species were differentially infected by water mites. I then determined if the relative size of geographical range of sibling host species was associated with relative parasitism levels and moreover whether the occasional patterns observed between gregarines and damselflies documented in Mlynarek et al. (2012) was also observed when considering ectoparasitic mites. More specifically, I was testing whether host species with smaller geographic range have higher levels of ectoparasitism, by analogy. I then assessed whether closely-related host species were infected by the same putative Arrenurus species using molecular techniques for parasite identification thereby making comparisons of parasitism between sibling host species more direct. Related to this objective, I was interested in whether the host species with higher measures of parasitism were also those with greater numbers of parasite species. In addressing these objectives, I also examined the extent to which Arrenurus water mite species show habitat or host species preferences. In summary, I was interested in whether a host species geographical range influenced measures of ectoparasitism and whether the patterns were repeatable or potentially affected by moderator variables (host species identity, habitat used). 


\section{METHODS}

\section{Specimen collection}

Ten species of Coenagrionidae (Argia moesta (Hagen) and A. violaceae (Burmeister); Enallagma boreale (Selys) and E. ebrium (Hagen); E. signatum (Hagen) and E. vesperum Calvert; Ischnura posita (Hagen) and I. verticalis (Say); and, Nehalennia gracilis Morse and $N$. irene (Hagen)) were collected belonging to five sibling species pairs in Coenagrionidae based on (sub)generic affiliations (Argia, Enallagma (Enallagma), Enallagma (Chromatallagma) subgenus, Ischnura and Nehalennia). I considered the two Enallagma species pairs as separate because they are considered to be in separate subgenera (May 2002). The particular host species were chosen because a second closely related host species was found in sympatry and because species in sibling species pairs differed in geographic range size (minimum of $100000 \mathrm{~km}^{2}$ difference between the two species in each pair, see Mlynarek et al 2012 and Chapter 2).

I collected adult damselflies using aerial sweep nets between 17 May and 15 July 2010 and 30 May and 15 July 2011. Damselflies in each species pair were collected from the same site at the same time (see below). The damselflies were stored in separate vials with $95 \%$ ethanol.

Species pairs were collected at different sites and times because host emergence varies temporally and spatially. Argia moesta and A. violaceae and E. (Chromatallagma) signatum and E. (Chromatallagma) vesperum were collected at the edge of Lake Opinicon $\left(44^{\circ} 33^{\prime} 56.32^{\prime} \mathrm{N}, 7^{\circ} 19^{\prime} 26.46^{\prime \prime} \mathrm{W}\right)$ on 30 June to 10 July 2010 ; the species $E$. 
(Enallagma) boreale and E. (Enallagma) ebrium were collected at Barb's Marsh (4431'27.54’N $\left.7^{\circ} 6^{\circ} 22^{\prime} 25.89^{\prime} \mathrm{W}\right)$ on 25 May, from 7 to 10 June 2010 and from 31 May to 21 June 2011, I. posita and I. verticalis were collected at a slow stream by Osprey Marsh (44'30'43.74”N, 76 23'39.32'W) on 4 and 10 July 2010, N. gracilis and N. irene were collected at Hebert Bog $\left(44^{\circ} 29^{\prime} 54.69^{\prime \prime} \mathrm{N}, 7^{\circ} 24^{\prime} 53.66^{\prime \prime} \mathrm{W}\right)$ on 7 and 30 June 2011 and from 6 June to 18 July 2011.

All individuals collected were inspected for parasitism by looking at ventral side of the thorax and abdomen. All Arrenurus spp. water mites were counted.

\section{Statistical measures of general parasitism}

Differences between sibling species in prevalence, the proportion of hosts infected with at least one larval Arrenurus, and intensity, the mean number of Arrenurus on only the infected hosts, was assessed in QP3.0 (Rózsa et al 2000). Prevalence estimates were provided with Clopper-Pearson 95\% confidence intervals (Zar 1999) whereas mean intensity estimates were provided with bootstrap confidence limits (Rózsa et al 2000).

More specifically, I compared difference in prevalence between species in each species pair using the chi-square test. To test for differences in intensity, I performed a Bootstrap 2-sample t-test (with 2000 bootstrap replicates) between species in each species pair. I, therefore, performed ten tests, five tests to test for differences in prevalence between species in five species pairs and five tests to test for differences in 
mean intensities between species in the five species pairs. Both series of statistical tests were done in QP3.0 (Rózsa et al 2000).

To explore any potential effects of geographical range on parasite prevalence or intensity, I simply inspected whether any significant differences between species in species pairs seemed to be predicted reliably by either the more or less widely distributed host species having either the higher or lower prevalence or intensity of Arrenurus spp. infection.

DNA barcoding of Arrenurus

To address whether host species of sibling species pairs might share putative mite species, and to have a preliminary account of host species range of those mite "species", DNA extractions from 62 larval mites was performed (from 70 larval water mites selected haphazardly, based principally on host species and site of host collection). This haphazard selection was as follows: five water mites were obtained from each host species from three different host individuals with mites coming from the thorax and/or abdomen (because thoracic and abdominal mites are often different species based on laboratory rearing of larval water mites through to adulthood (Bruce Smith, pers. comm.). All ten host species had thoracic mites. Four of ten species also had abdominal mites. In those latter species, both thoracic and abdominal water mites were collected, which is why sample size was initially 70 .

Total genomic DNA was extracted from whole specimens for 24 hours using a DNeasy Tissue kit (Qiagen Inc., Santa Clara, CA, USA). Following extraction, mites were removed from the extraction buffer, and genomic DNA was purified following the 
DNeasy Tissue kit protocol. PCR amplifications were performed following the protocol of Mlynarek et al. (2014), amplification cycles were performed on an Eppendorf ep Gradient S Mastercycler (Eppendorf AG, Hamburg, Germany). Primer pairs Alt-ALF1 (5'-GCDTGRTCWGGRATAGTDGGAGCMAG-3') + $\quad+\quad$ Alt-ALR1 GACCCRGCYGGAGGDGGRG -3'), and LCO1490 + HCO2198 (Folmer et al 1994) were used to attempt to amplify a $608 \mathrm{bp}$ and $708 \mathrm{bp}$ fragment, respectively, of the 5 '-end of COI of Arrenurus larvae and not their damselfly hosts. The thermocycler protocol for COI amplification was as follows: initial denaturation cycle at $94^{\circ} \mathrm{C}$ for $3 \mathrm{~min}$, followed by 45 cycles of $94^{\circ} \mathrm{C}$ for $45 \mathrm{~s}$, primer annealing at $45^{\circ} \mathrm{C}$ for $45 \mathrm{~s}, 72^{\circ} \mathrm{C}$ for $1 \mathrm{~min}$, and a final extension at $72^{\circ} \mathrm{C}$ for $5 \mathrm{~min}$.

Amplified products and negative controls were visualized on $1 \%$ agarose electrophoresis gels, and purified using pre-cast E-Gel CloneWell $0.8 \%$ SYBR Safe agarose gels (Invitrogen, Carlsbad, CA, USA) following Gibson et al. (2010). Sequencing reactions followed the protocol of Knee et al. (2012), and sequencing was performed at the Agriculture \& Agri-Food Canada, Eastern Cereal and Oilseed Research Centre Core Sequencing Facility (Ottawa, ON, Canada).

Sequence chromatograms were edited and contiguous sequences were assembled using Sequencher v4.7 (Gene Codes Corp., Ann Arbor, MI, USA). COI sequences were aligned manually in Mesquite v2.74 (Maddison and Maddison 2010) according to the translated amino acid sequence. Sequences have been submitted to GenBank (KF880845-KF880906). Homologous sequences from eight Arrenurus sp. individuals 
collected from Nehalennia gracilis and N. irene damselflies (Mlynarek et al. 2012) were included in the COI alignment, and seven water mite species were selected from GenBank to serve as outgroups (AB530314, JX838402, JX836526, JX835088, JN018105, JN018109, AB530311).

Pairwise distances were calculated using neighbour-joining analysis with the uncorrected ("p") model in PAUP* v4.0b10 (Swofford 2003). Phylogenetic analysis of the COI dataset was performed using Bayesian inference (BI) in MrBayes v3.1.2 (Huelsenbeck and Ronquist 2001, Huelsenbeck and Ronquist 2003). The best-fit model of molecular evolution was determined to be $\mathrm{GTR}+\mathrm{I}+\mathrm{G}$, using MrModeltest v2.3 (Nylander 2004). Bayesian analysis was performed in MrBayes with a Markov Chain Monte Carlo (MCMC) method, two independent runs, with nucmodel $=4 \mathrm{by} 4, \mathrm{~N}_{\mathrm{st}}=6$, rates $=$ invgamma, samplefreq $=1000$, four chains $=$ one cold and three heated, 10 million generations. In Mesquite, the remaining trees, excluding the burn-in (100), were used to generate a majority-rule consensus tree displaying the posterior probability supports for each node. Bayesian analysis was performed using the Cyberinfrastructure for Phylogenetic Research (CIPRES) portal (Miller et al. 2010). In TNT v1.1 (Goloboff et al. 2008) node support was assessed using jackknife resampling with $36 \%$ of characters removed and 1000 replicates, using a heuristic search with tree bisection-reconnection (TBR) branch swapping and 1000 random addition sequence replicates. 
I used the Bray-Curtis dissimilarity index to determine whether water mite species richness and composition differed more between or within host species pairs in $\mathrm{R}$ using vegdist in the package VEGAN (Oksanen et al. 2013).

\section{RESULTS}

\section{Measures of general Arrenurus parasitism}

In total, 1162 damselflies were collected across four sites in Southeastern Ontario, within a 20-km radius of the Queen's University Biological Station (Table 3.1).

Prevalence of Arrenurus varied considerably between host species and species pairs (Table 3.1). Overall prevalence varied between 4\% in Argia moesta and 54\% in Enallagma vesperum. Both of those species were collected from Lake Opinicon. Significant or near significant differences in prevalence between species in sibling species pairs were found in two of five comparisons (Fig. 3.1). In particular, there was a significant difference in prevalence between the species of Enallagma (Chromatallagma) $\left(\chi^{2}=16.57 \mathrm{df}=1 \mathrm{p}<0.001\right)$ where $54 \%$ of Enallagma vesperum individuals were infected compared to $27 \%$ of E. signatum. Ischnura posita had a prevalence of $5 \%$ whereas $I$. verticalis had a prevalence of $17 \%$. The difference in prevalence in these species pair was close to significant $\left(\chi^{2}=3.58 \mathrm{df}=1 \mathrm{p}=0.06\right)$. In comparison, no significant differences in prevalence were found for Argia, Enallagma subgenus Enallagma and Nehalennia (Table $3.2)$.

The data on mean intensity of infection told a similar story (Fig. 3.2). Overall, the minimum mean intensity was 1.5 in $A$. moesta and the maximum was 11.9 in Enallagma 
ebrium (Table 3.1). In Enallagma (Enallagma), Enallagma boreale was infected by an average of 4.6 mites per infected host individual, but E. ebrium was infected by an average of 10.2 mites per individual ( $\mathrm{t}=-2.65$ bootstrap $\mathrm{p}=0.01)$. In Argia, Enallagma (Chromatallagma), Ischnura and Nehalennia no significant differences in intensity were found between the species (Table 3.1).

At least two species pairs showed no differences in either prevalence or mean intensity of infection and yet sibling species differed in geographical range (Argia and Nehalennia). The other three species pairs had a significant or near significant difference in prevalence or a significant difference in intensity. In Enallagma (Chromatallagma), E. vesperum had significantly higher prevalence of parasitism: this species has the smaller geographic range of the pair $\left(2.60 \times 10^{6} \mathrm{~km}^{2}\right.$ compared to $3.26 \times 10^{6} \mathrm{~km}^{2}$ in E. signatum; Table 3.1). Similarly, in Enallagma (Enallagma), E. ebrium had significantly higher intensity of parasitism; E. ebrium is less widely distributed $\left(4.35 \times 10^{6} \mathrm{~km}^{2}\right)$ than $E$. boreale $\left(6.53 \times 10^{6} \mathrm{~km}^{2}\right.$; Table 3.1$)$. In Ischnura, I. verticalis has both nearly significant higher prevalence of water mite infection and larger geographic range than I. posita (4.36 x $10^{6} \mathrm{~km}^{2}$ compared to $3.13 \times 10^{6} \mathrm{~km}^{2}$; Table 3.1 ). Thus in $60 \%$ of cases, sibling host species with differences in size of geographic range differed in either prevalence or intensity of parasitism, but in those cases the actual differences are inconsistently related to relative size of the pair member's geographic range. 
Chapter 3

Table 3.1. Details of parasitism in ten species of damselflies sampled at four sites. $\mathrm{N}$ represents the number of individuals of each species sampled. Sample size (N) with the number of individuals infected (N. inf), geographic range (Range) of the host and number of Operational Taxonomic Units (OTUs) are provided. Confidence limits for prevalence and intensity are shown respectively.

\begin{tabular}{|c|c|c|c|c|c|c|}
\hline Host species & Location & $\begin{array}{c}\mathrm{N} \\
(\mathrm{N} \text { inf.) }\end{array}$ & $\begin{array}{c}\text { Range } \\
\left(10^{6} \mathrm{Km}^{2}\right)\end{array}$ & OTUs & $\begin{array}{c}\text { Prevalence } \\
(95 \% \mathrm{CI})\end{array}$ & $\begin{array}{l}\text { Intensity } \\
(95 \% \mathrm{CI})\end{array}$ \\
\hline Argia moesta & $\begin{array}{c}\text { Lake } \\
\text { Opinicon }\end{array}$ & $90(4)$ & 4.10 & 1,7 & $\begin{array}{c}0.04 \\
(0.01-0.1)\end{array}$ & $\begin{array}{c}1.50 \\
(1-1.7)\end{array}$ \\
\hline A. violaceae & $\begin{array}{c}\text { Lake } \\
\text { Opinicon }\end{array}$ & $97(8)$ & 3.98 & 1,9 & $\begin{array}{c}0.08 \\
(0.04-0.2)\end{array}$ & $\begin{array}{c}5.75 \\
(1.4-18.1)\end{array}$ \\
\hline Enallagma boreale & Barb Marsh & $57(15)$ & 6.53 & 4,5 & $\begin{array}{c}0.26 \\
(0.2-0.4)\end{array}$ & $\begin{array}{c}4.60 \\
(2.9-7.4)\end{array}$ \\
\hline E. ebrium & Barb Marsh & $106(31)$ & 4.35 & 4,5 & $\begin{array}{c}0.29 \\
(0.2-0.4)\end{array}$ & $\begin{array}{c}10.16 \\
(6.8-13.7)\end{array}$ \\
\hline E. signatum & $\begin{array}{c}\text { Lake } \\
\text { Opinicon }\end{array}$ & $120(32)$ & 3.23 & 1,2 & $\begin{array}{c}0.27 \\
(0.2-0.4)\end{array}$ & $\begin{array}{c}9.81 \\
(5.2-22.1)\end{array}$ \\
\hline E. vesperum & $\begin{array}{c}\text { Lake } \\
\text { Opinicon }\end{array}$ & $101(54)$ & 2.6 & 1 & $\begin{array}{c}0.54 \\
(0.4-0.6)\end{array}$ & $\begin{array}{c}11.35 \\
(7.0-18.9)\end{array}$ \\
\hline Ischnura posita & $\begin{array}{l}\text { Osprey } \\
\text { Marsh }\end{array}$ & $77(4)$ & 3.13 & $3,4,6$ & $\begin{array}{c}0.05 \\
(0.01-0.1)\end{array}$ & $\begin{array}{c}3.25 \\
(1.0-5.5)\end{array}$ \\
\hline I.verticalis & $\begin{array}{l}\text { Osprey } \\
\text { Marsh }\end{array}$ & $23(4)$ & 4.36 & $1,3,6$ & $\begin{array}{c}0.17 \\
(0.1-0.4)\end{array}$ & $\begin{array}{c}6.75 \\
(1.5-16.5)\end{array}$ \\
\hline Nehalennia gracilis & Hebert Bog & $208(25)$ & 1.51 & 8 & $\begin{array}{c}0.12 \\
(0.1-0.2)\end{array}$ & $\begin{array}{c}2.12 \\
(1.6-3.4)\end{array}$ \\
\hline$N$. irene & Hebert Bog & $284(45)$ & 4.03 & 8 & $\begin{array}{c}0.16 \\
(0.1-0.2)\end{array}$ & $\begin{array}{c}1.78 \\
(1.4-2.8)\end{array}$ \\
\hline
\end{tabular}


Table 3.2. Results of the Chi-square test for comparing prevalence and Bootstrap t-test for mean intensity of parasitic infection within the five Zygoptera species pairs. Significant or close to significant values are in bold

\begin{tabular}{lccccc}
\hline Host species pair & \multicolumn{3}{c}{ Prevalence } & \multicolumn{2}{c}{ Intensity } \\
& $\chi^{2}$ & $\mathrm{df}$ & $\mathrm{p}$ & t-value & Bootstrap p \\
\hline Argia & 1.12 & 1 & 0.29 & 1.05 & 0.41 \\
Enallagma E & 0.16 & 1 & 0.69 & 2.65 & $\mathbf{0 . 0 1}$ \\
Enallagma $C$ & 16.57 & 1 & $<\mathbf{0 . 0 1}$ & 0.33 & 0.35 \\
Ischnura & 3.58 & 1 & $\mathbf{0 . 0 6}$ & 0.66 & 0.54 \\
Nehalennia & 1.44 & 1 & 0.52 & 0.70 & 0.51 \\
\hline
\end{tabular}




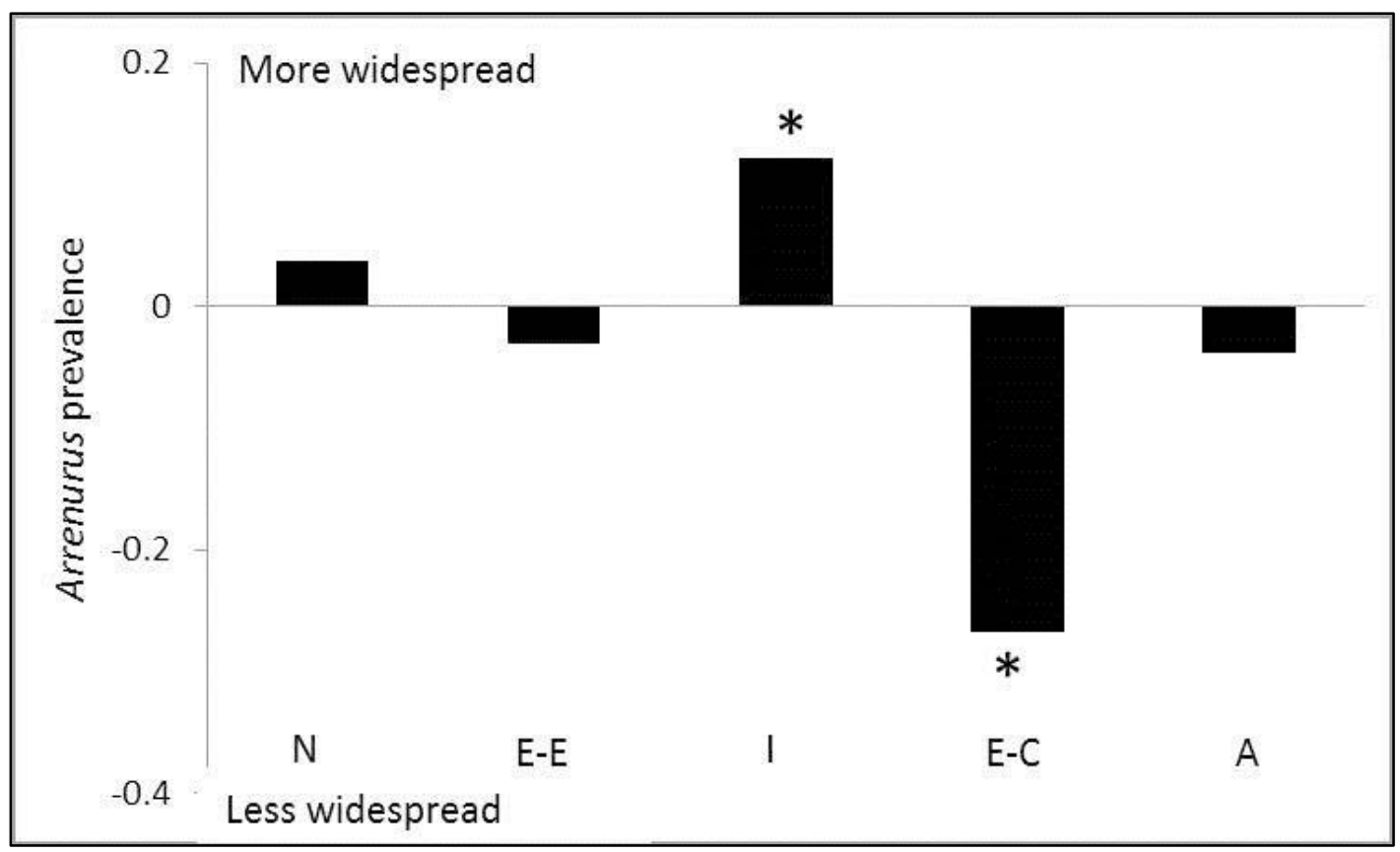

Figure 3.1. Within species pair difference in measures of prevalence between species. A positive difference means that the more widespread species of the species pair has higher estimate of prevalence of infection (from Table 3.1); a negative difference means that the less widespread species has higher estimate of prevalence. Actual significant differences in prevalence values are marked by $*$. The organization of the species pairs reflects geographical range differences within the species pair from most different to least different. Key: A, Argia; E-C, Enallagma Chromatallagma; E-E, Enallagma Enallagma; I, Ischnura; N, Nehalennia. 


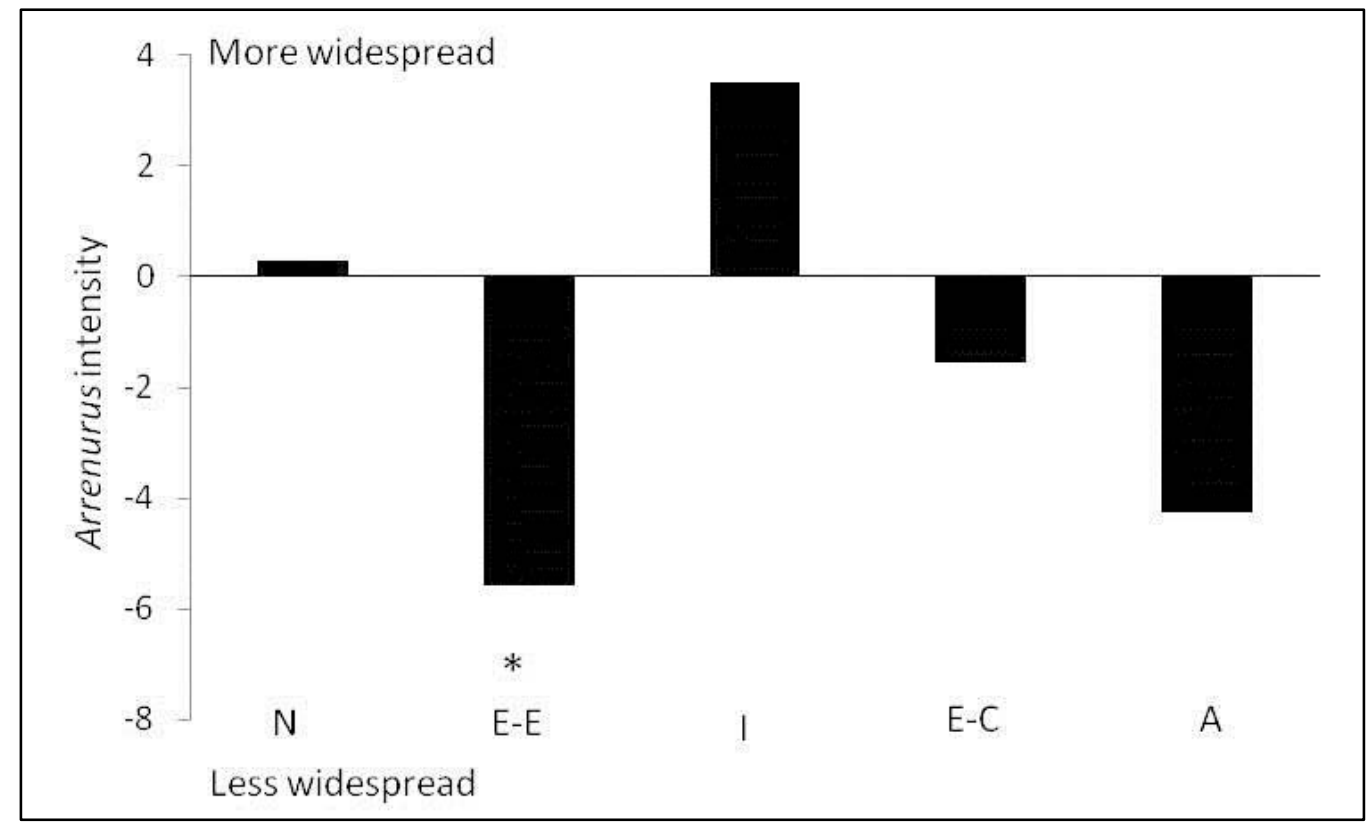

Figure 3.2. Within species pair difference in measures of water mite mean intensity between species. A positive difference means that the more widespread species of the species pair has higher estimate of intensity of infection (from Table 3.1); a negative difference means that the less widespread species has higher estimate of intensity. Actual significant differences in prevalence values are marked by $*$. The organization of the species pairs as in Figure 3.1. 


\section{DNA Barcoding}

COI was amplified from 62 water mites, with 587 characters in total, 296 constant, 50 parsimony-uninformative and 241 parsimony-informative. Mean base pair frequencies (A: $0.31574, C: 0.21282, \mathrm{G}: 0.13819, \mathrm{~T}: 0.33325)$ were found to be homogenous across all specimens $\left(\chi^{2}=52.66, \mathrm{df}=207 P=1.0\right)$.

Bayesian inference $(\mathrm{BI})$ of the $\mathrm{COI}$ dataset was performed for 10 million generations, producing 19802 trees (after burn-in), which were summarized in a majority rule consensus tree $(\mathrm{TL}=1048, \mathrm{CI}=0.4599, \mathrm{RI}=0.8617)($ Fig. 3.3 $)$. The BI consensus tree was well supported, with most nodes having moderate to high posterior probabilities and jackknife support (Fig. 3.3). The ingroup are divided into nine well-supported clades, hereafter referred to as Operational Taxonomic Units (OTUs). Average interspecies divergence was $15 \% \pm 1.1(5-21 \%)$, and average intraspecies divergence was $0.7 \% \pm 0.3$ (0-4\%). Average divergence between putative species 1, 2 and 3 was $9 \% \pm 1$, divergence between species 4 and 5 was $6 \% \pm 0.3$, between 6 and 7 was $10 \% \pm 0.3$, and species 8 and 9 show $8 \% \pm 0.3$ divergence. Considering the high level of divergence between putative species and the low divergence within each species, as well as the strong support for each species in the phylogenetic reconstruction, it appears that each putative species may be distinct. However, I do not advocate the notion that these are new species, distinguishing between species using pairwise distances from a fragment of a single gene is not a reliable taxonomic approach (Cognato 2006).

Based on my Bayesian tree (Fig. 3.3), I found in Argia that there are three Arrenurus OTUs that infect Argia moesta and $A$. violaceae. OTU 9 is specific to $A$. 
violaceae and OUT 7 to A. moesta (Fig. 3.3). Each of these OTUs is associated with a single host species; therefore I consider them to be candidates for specialist parasites. OTU 1 was collected both A. moesta and A. violaceae, and also two other Enallagma species (E. signatum and E. vesperum) from lake Opinicon indicating that this mite has a broad host species spectra (Fig. 3.4). In addition, there is one record of this mite OTU present on Ischnura verticalis in a marsh habitat. Interestingly, this broad host generalist was the only OTU collected from E. vesperum (out of 5 mites sampled). Thus, I discovered that mites of two host species pairs (examined first) shared a mite species (Fig. 3.3). 


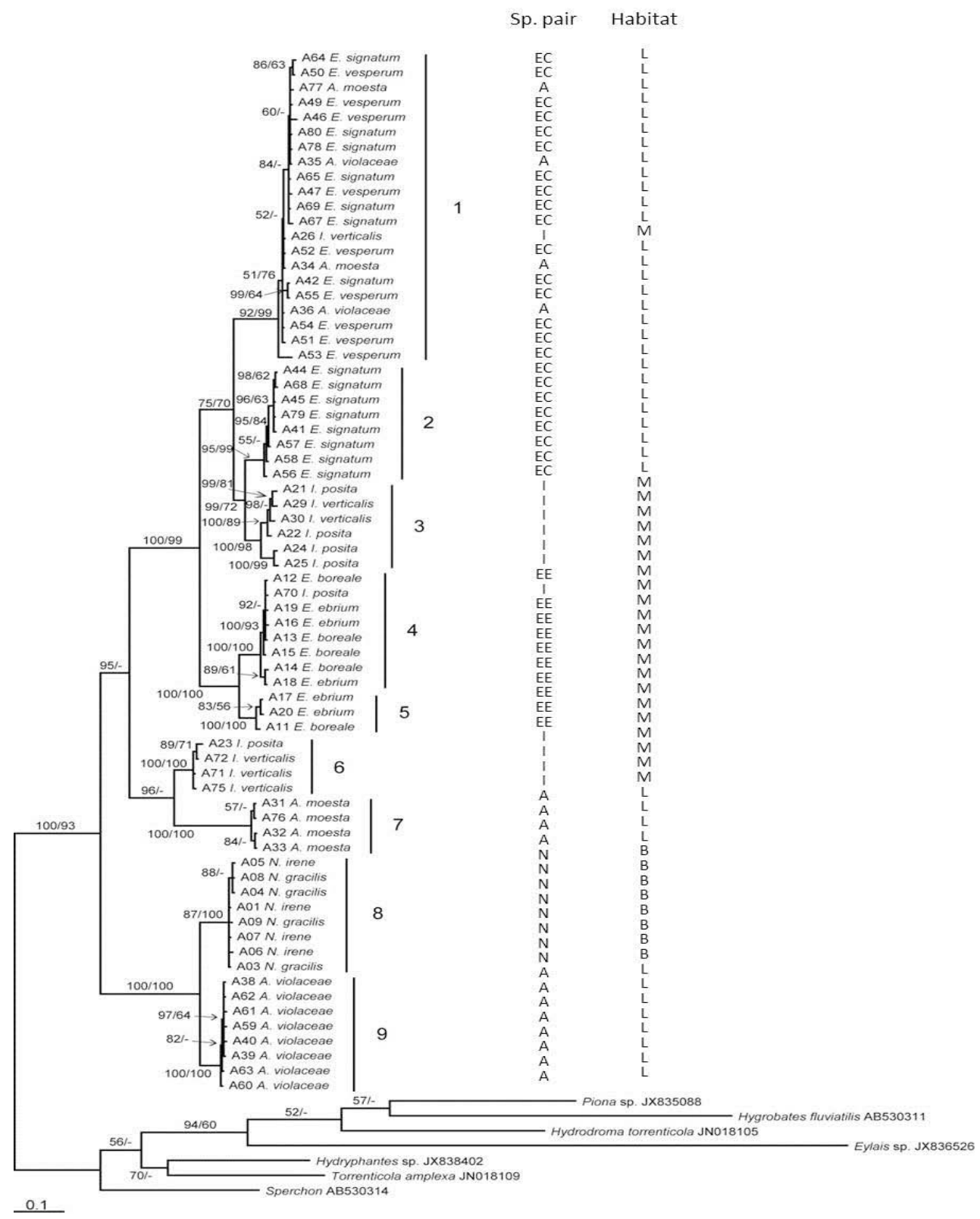

Figure 3.3. Majority rule consensus tree of 19802 trees generated by Bayesian MCMC analysis (10 million generations) of 587bp fragment of COI from 77 water mites, 70 ingroup and 7 outgroup specimens $(\mathrm{TL}=1048, \mathrm{CI}=0.4599, \mathrm{RI}=0.8617)$ posterior probability $>50 \%$ / jackknife support. Species pair A=Argia; EE=Enallagma Enallagma $; \mathrm{EC}=$ Enallagma Chromatallagma $; \mathrm{I}=$ Ischnura $; \mathrm{N}=$ Nehalennia, habitat type $\mathrm{B}=\mathrm{Bog} ; \mathrm{L}=$ Lake, $\mathrm{M}=$ Marsh. 
Similarly, the two Enallagma (Enallagma) host species were both infected by two closely related OTUs ( $4 \& 5$ ). These OTUs are distinct, based on sequence divergence and branch support, but are closely related to one another (Fig. 3.3). Therefore these two OTUs were collected from at least these two species of damselflies. However, I could not determine whether these two parasite species were present on other host species. Again, it appears that sibling species share mite species. Ischnura is an interesting species pair in that four different OTUs have been collected from these hosts, despite the limited sampling. OTU 3 and 6 were collected from both Ischnura species again supporting sharing of parasites. But OTU 1, the aforementioned generalist on damselfly host species in the lake, was found on one Ischnura verticalis individual from a marsh inlet site. Additionally, OTU 4 (Fig. 3.3) infecting I. posita is shared with Enallagma (Enallagma) damselfly hosts from a different marsh. Finally, both Nehalennia species collected in the bog are infected by OTU 8 (Mlynarek et al 2014). The results show that the sharing of mite species by sibling species of damselflies (and beyond those host clades) is a common occurrence (Fig. 3.4). Species richness and composition within the host species pair did not differ (range of Bray-Curtis dissimilarity Index varied between 0.00 in Nehalennia to 0.86 in Argia) as much as between species pairs where the Bray-Curtis dissimilarity index ranged between $0.79-1.00$. The dissimilarity index on 0.79 was determined between $A$. violaceae and E. vesperum, which share water mite OTUs (Fig. 3.4). Figure 3.3 is also annotated with whether or not the host and mites were collected from a lake, marsh or bog. It appears that mite species are restricted to one type of habitat but this is not a "hard and fast" rule. 


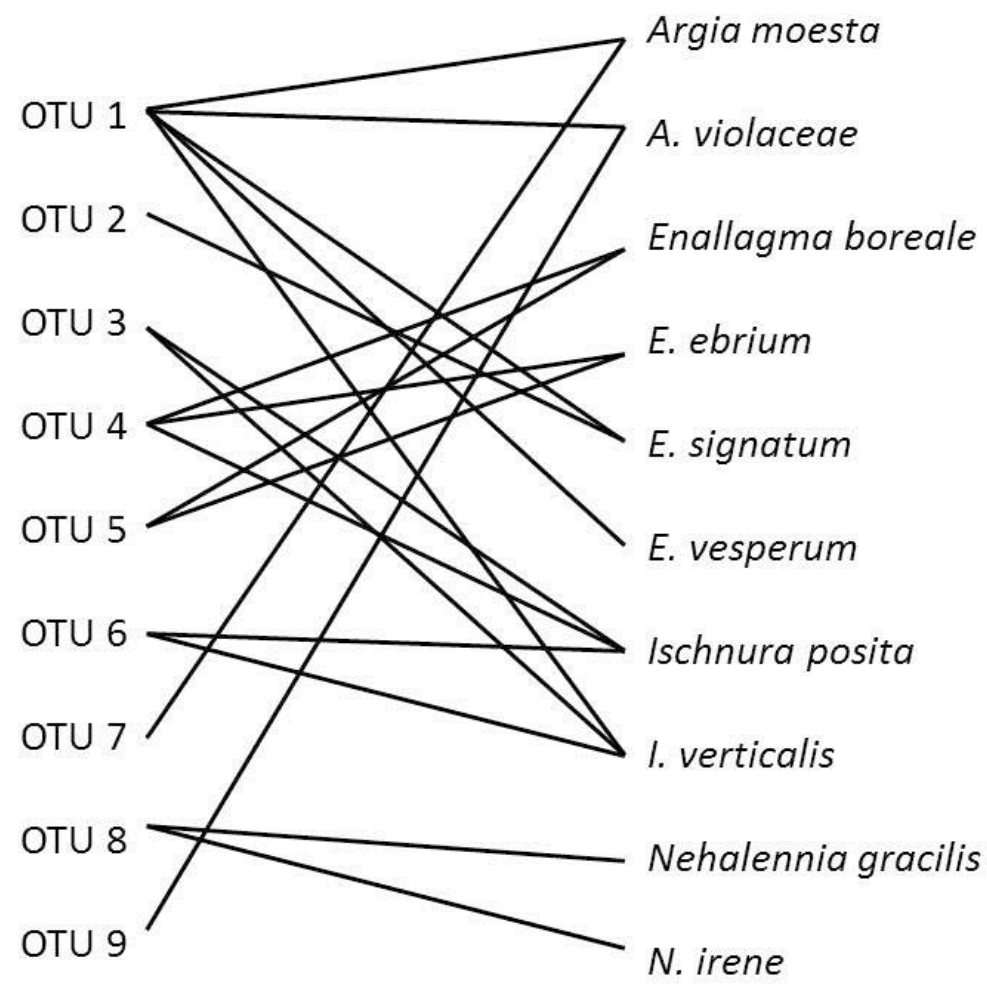

Figure 3.4. Schematic network of damselfly-water mite OTUs. The solid lines represent associations between a damselfly host species and a water mite OTU. 


\section{DISCUSSION}

Relative geographic range size did predict relative prevalence of parasitism in some species pairs, but not reliably across all species pairs. To reiterate, there were significant differences in prevalence for Enallagma (Chromatallagma) species pair where the less widespread species had a higher prevalence of Arrenurus spp. In one instance (Ischnura species pair) did the more widespread host species, I. verticalis, have significantly higher intensity of Arrenurus than the more geographically restricted host species I. posita. Even referring to these two comparisons, geographic range is not a reliable predictor of parasitism in these types of insect host-invertebrate parasite associations. Across all other species pairs, differences in geographic range were evident, but no species differences in relative prevalence of ectoparasitism was observed. And furthermore, in only in the Enallagma (Enallagma) species pair, did the less widespread species have a higher mean intensity of infection by Arrenurus than the more widespread Enallagma species. Across all other species pairs, there were no species differences in mean intensity of mite parasitism.

In comparison to the findings in this study, Tella et al. (1999) tested other factors that could potentially mask the effect of host geographic range such as sampling effort, vector availability and embryonic development time, but the researchers concluded that hematozoan prevalence appears determined by host geographic range. Mlynarek et al. (2012) also reported that measures of gregarine parasitism were higher in the less widespread species pair in four out of the seven damselfly species pairs. Additional comparisons with this study demonstrate that the species pairs respond differently, 
depending on the type of parasite studied. For example, in Mlynarek et al (2012) Ischnura posita had a significantly higher prevalence of gregarines than its more widespread counterpart, Ischnura verticalis. In this study, Ischnura verticalis has a near significant higher prevalence of Arrenurus water mites than I. posita. Relative size of a host's geographic range, therefore, can have different or no effect on relative parasitism, depending on the type of parasite studied.

Based further on Mlynarek et al. (2012) and this study, 12 species comparisons were done with respect to prevalence or intensity of either gregarines or mites. In half of the 12 species pair comparisons, there were statistical differences between species in either or both prevalence and intensity of either gregarine or mite infections. In four of these cases, the less widespread host species had higher prevalence and/or intensity of the parasite of interest whereas in the two remaining cases, it was the more widely distributed host with the higher parasite levels (prevalence of gregarines for Nehalennia and prevalence of mites for Ischnura). In one case, Enallagma Chromatallagma, there were significant differences in both prevalence of mites and prevalence of gregarines whereby the less widespread host had higher prevalence of parasites. Stated another way, in four $(57 \%)$ of the seven comparisons involving gregarines and in three $(60 \%)$ of the five comparisons involving mites, closely related host species differed in one or more measures of parasitism. In the remaining $40 \%$ of the cases, the closely related species did not differ in measures of parasitism despite showing differences in geographical range. Further, in one (25\%) of four comparisons involving significant differences in gregarines, it was the more widely distributed host that had higher gregarine prevalence. A similar 
result was found where one (33\%) of three comparisons (Ischnura) involving significant differences in mite intensities, was attributed to the species with the greater geographic range.

In this study, using COI barcodes, there are nine OTUs within Arrenurus infecting the ten damselfly species. Arrenurus OTU 2, 7 and 9 appear specialized on a specific damselfly host species (E. signatum, A. moesta and A. violaceae), but sampling was limited. OTU 1 infects four host species in lakes. The Arrenurus OTUs 3,4,5,6 and 8, collected in the marsh and bog, infect at least two species each. Water mites appear affiliated with a habitat and not so much specialized on phylogenetically related host species. Similarly to Krasnov et al. (1997), habitat was a strong factor influencing flea parasitism on rodents in the Negev desert. As well, in my case and that of Krasnov et al (2006), both host species' and habitat characteristics are important in determining parasite species composition. Except for OTU 1, collected from an I. verticalis host, all the water mites are restricted to one particular habitat type. A potential reason could be that this I. verticalis individual was a vagrant into the marsh where it was collected, or that this mite is found in many habitats, mostly in lakes and rarely in others. The important point is that comparisons of parasitism between sibling species based on broad taxonomic identification of the parasites is somewhat permitted because all sibling host species share at least one mite species. If there was some strong interaction between a host geographic range and the intensity of interactions with given parasite species, it should be detected using even broad taxonomic comparisons, where only mites are being compared and not every parasite is identified to species. 
There is a different pattern when comparing a group of parasites without considering broad taxonomic identification. When considering parasite species richness, it is expected that the more widespread hosts have a higher richness of parasite species (Dritschilo et al. 1975). In cricetid rodents, host geographic range was strongly associated with Demodex mite species richness (Dritschilo et al. 1975). In waterfowl, Gregory (1990) demonstrated a clear positive relationship between host geographic range and parasite species richness, using cross-species comparisons. In European fresh-water fish, Simková et al. (2001) concluded that host geographic range influenced parasite species richness through its effect on host local abundance and occurrence. Their research was based on 39 fish host species and a comprehensive assessment of internal and external parasites. In my case, I did not see this occurring but only can report on nine mite species across five pairs of damselfly host species. Notwithstanding, host species in each pair have a comparable number of mite species infesting them, even though certain host species might have species-specific parasites. The only exception in my study was Enallagma (Chromatallagma) species pair where the more widespread E. signatum has two Arrenurus species (one host specific) whereas E. vesperum, the more restricted species, is only infected by one Arrenurus species. Even here, the conclusion that one host species has a higher parasite species diversity has to be met with scepticism, especially given that, when controlling for site, only one out of six instances does the more widespread species show this tendency toward higher parasite species richness.

In this study, I documented host- parasite associations in multiple species pairs. I observed that there appear to be varying degrees of specificity in host range of Arrenurus 
mites. In conjunction with the findings of Mlynarek et al. (2012) on internal parasites of damselflies, my findings suggest that host species' geographic range size does not reliably explain relative species differences in measures of parasitism between closely related host species. Water mites species found in one type of habitat tend not to be present in another type of habitat but can parasitize few to many host species found in a habitat. More work is needed to further elucidate Arrenurus species boundaries, using additional molecular markers or rearing larvae for adult identification. Further work is needed on determining host species use before I can test the importance of other ecological factors (e.g. phenology or regional representation of both hosts and parasites) on past and ongoing evolution of these associations. 


\section{CHAPTER FOUR}

\section{Host phenology, geographic range size and regional representation explain interspecific variation in damselfly-water mite associations.}

This chapter formed the basis for the following manuscript submitted to Ecography

Mlynarek, J.J., Knee, W, and Forbes, M.R. Host phenology, geographic range size and regional representation explain interspecific variation in damselfly-water mite associations. 


\section{ABSTRACT}

Species interactions are determined by ecological and evolutionary factors. One of the central goals of evolutionary parasitology is to explain variation in parasitism between closely related host species. In this study, I tested which host species' characteristics explain the nature and level of parasitism for host damselfly (Coenagrionidae) -water mite (Arrenuridae) parasite associations. Prevalence and intensity of mite parasites, and mite species richness were examined in relation to geographic range size, regional representation, relative local abundance, phenology and body size of host damselfly species. A total of 7107 damselfly individuals were collected representing 16 species from 13 sites in Southeastern Ontario and Southwestern Quebec, Canada. Using comparative methods, differences in prevalence and intensity of parasitism could be predicted by a host species' geographic range and phenology. Barcoding based on Cytochrome Oxidase I revealed 15 operational taxonomic units (OTUs) for mite species. The number of mite OTUs known to infest a given host species was explained by a host species' regional representation. My findings demonstrate the need to measure factors at several ecological scales in order to understand the breadth of evolutionary interactions with host-parasite associations and the selective 'milieu' for particular species of both hosts and parasites. 


\section{INTRODUCTION}

Evolutionary history of the association between two species is expected to have an effect on present-day interactions, but so are ecological factors (Thompson 2005). In most cases, that explanatory power of ecological factors depends on the host-parasite system under study, perhaps because of certain life history traits (Poulin 2007). Several ecological factors have been reported to explain variation in parasitism between host species, such as size or landscape configuration of habitat (Tella et al. 1999) : size, age, reproductive stage or phenology of the host species (Tripet and Richner 1997, Arneberg et al. 1998, Krasnov et al. 2006, Munoz et al. 2007), and size, virulence and life history of the parasite (Krasnov et al. 2004). Host species' characteristics such as geographic range size, regional representation, local abundance, and phenology have been offered as explanations for interspecific variation in parasitism (Morand and Krasnov 2010). As a host species range increases, so does the number of species it interacts with (Krasnov et al. 2004, Ilvonen et al. 2011) because the host comes into contact with more potential parasite species (Price et al. 1988). However within its entire range, the host species is not evenly distributed in either space or time (Thompson 2005), because there are habitats where individuals cannot survive and reproduce well, if at all.

Thus, not only the size of a species geographic range, but also actual representation of host species across the region of interest could be important. It is expected that a more regionally represented host species will come into contact with more parasite species than host species less well represented regionally (cf. Durrer and 
Schmid-Hempel 1995). Species are not only unevenly distributed across space, but there are also differences in the representation of a species through time (phenology). These phenological differences between species are also expected to influence their interactions, especially between groups that are active during different times of the year (Altizer et al. 2006, Locklin and Vodopich 2010). One expectation is that density of infective stages of parasites would follow density of potential hosts throughout the season of activity (Forbes et al. 2012). More specifically, the density of infective stages of parasites and measures of parasitism itself (e.g. prevalence and intensity) should be highest when potential host individuals of one or more species are most abundant (Yourth et al 2002). Local abundance of a host species might influence its interactions with co-occurring species. The intensity of interactions increases with increased host abundance because the probability of encounters increases (Poulin 1991, Arneberg et al. 1998, Krasnov et al. 2002, Patterson et al. 2008). As a host species becomes more abundant in a habitat, it is expected to be under higher pressure from the parasites (Arneberg et al. 1998).

Parasite associations with insect hosts are not as well understood as those in vertebrate hosts. In mites associated with ants, mite prevalence was explained by the sociality, the size and the generic identity of the host species (Campbell et al. 2013). Durrer and Schimd-Hempel (1995) did a comprehensive study of bumblebee parasites and found that parasite load was positively correlated with average colony size, local abundance and regional representation of the host. The geographic range of damselfly hosts explained in part the prevalence and intensity of gregarine endoparasites (Mlynarek et al. 2012). In contrast, host geographic range was a poor predictor of Arrenurus water 
mite prevalence and intensity when comparing pairs of closely related damselfly host species from single sites (Mlynarek et al. 2013).

The association between damselflies and Arrenurus water mites is nonetheless a good model system for host-parasite studies because of the nature of the obligate interaction characterized by a direct life cycle, the similarity in trophic level of the host species, and the known differences in range and expected differences in phenology and regional representation of different host species (Forbes and Robb 2008). Larval Arrenurus mites contact their future damselfly hosts in the water column. As the formerly nymphal damselfly ecloses, the mites move from the exuviae onto the teneral adult and embed their gnathosome through the unsclerotized cuticle into the hemolymph (Smith et al. 2010). The mites then form a blind ended feeding tube known as a stylostome and start feeding (Smith et al. 2010). Damselflies can resist mites by grooming loosely attached mites, or through melanisation once a mite has formed a stylostome (Leung et al. 1999, Forbes et al. 1999, Mlynarek et al. 2014).

In this study, I tested the extent to which geographic range, regional representation, local abundance, phenology and body size of host species are good predictors of prevalence, and intensity of water mite parasitism and, predictors of proxies of species richness of mite parasites. My study was restricted to damselflies of the family Coenagrionidae, but I expand upon Mlynarek et al. (2013) by examining more host species across many sites and by using parasite species diversity as an additional measure of parasitism. I tested the generality of regional representation, local abundance and body 
size in explaining interspecific variation in parasitism, because they have been shown to be important factors in vertebrate-parasite systems (Poulin 2007).

\section{METHODS}

\section{Sites}

Thirteen sites were chosen and were visited on a weekly basis for eight weeks in June and July 2011 (Fig. 4.1). Sites were spread along a $400 \mathrm{~km}$ longitudinal transect from the Queen's University Biological Station (QUBS), 60 km Northwest of Kingston, Ontario to Johnville Bog $20 \mathrm{~km}$ east of the city of Sherbrooke, Quebec in hopes to maximize the numbers of species interactions occurring (Table 4.1). The most western sites (QUBS) were collected at the beginning of the week with the eastern sites collected at the end of the week. 


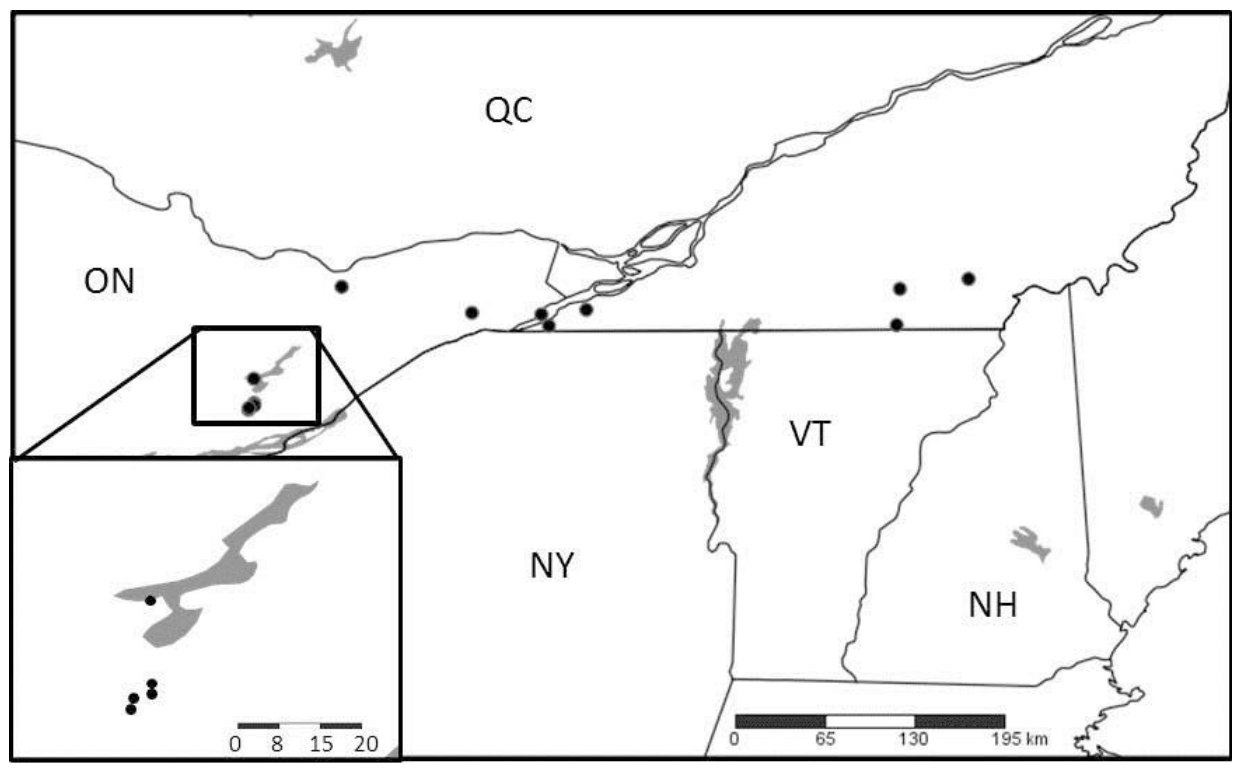

Figure 4.1. Map of study sites included in this study in Southeastern Ontario and

Southwestern Quebec.

Table 4.1. Study sites and their coordinates included in this study in Southeastern Ontario and Southwestern Quebec.

\begin{tabular}{lllll}
\hline Site & Province & Site Type & Latitude & Longitude \\
\hline Johnville & Quebec & Bog & $45^{\circ} 20^{\prime} 38.67^{\prime \prime N}$ & $71^{\circ} 44^{\prime} 18.17^{\prime \prime} \mathrm{W}$ \\
Marlington & Quebec & Bog & $45^{\circ} 2^{\prime} 44.39^{\prime \prime} \mathrm{N}$ & $72^{\circ} 12^{\prime} 16.98^{\prime \prime} \mathrm{W}$ \\
Large Teafield & Quebec & Bog & $45^{\circ} 8^{\prime} 4.02^{\prime \prime} \mathrm{N}$ & $74^{\circ} 13^{\prime} 0.47^{\prime \prime} \mathrm{W}$ \\
Newington & Ontario & Bog & $45^{\circ} 7^{\prime} 21.68^{\prime \prime} \mathrm{N}$ & $74^{\circ} 57^{\prime} 55.27^{\prime \prime} \mathrm{W}$ \\
Hebert & Ontario & Bog & $44^{\circ} 29^{\prime} 54.69^{\prime \prime} \mathrm{N}$ & $76^{\circ} 24^{\prime} 53.66^{\prime \prime} \mathrm{W}$ \\
Westport & Ontario & Bog & $44^{\circ} 41^{\prime} 57.69^{\prime \prime} \mathrm{N}$ & $76^{\circ} 22^{\prime} 46.55^{\prime \prime} \mathrm{W}$ \\
Lac aux Cerises & Quebec & Marsh & $45^{\circ} 16^{\prime} 51.00^{\prime \prime} \mathrm{N}$ & $72^{\circ} 10^{\prime} 9.34^{\prime \prime W}$ \\
Cooper & Ontario & Marsh & $45^{\circ} 6^{\prime} 59.04^{\prime \prime} \mathrm{N}$ & $74^{\circ} 30^{\prime} 51.59^{\prime \prime} \mathrm{W}$ \\
Pointe Leblanc & Quebec & Marsh & $45^{\circ} 2^{\prime} 12.18^{\prime \prime} \mathrm{N}$ & $74^{\circ} 27^{\prime} 56.70^{\prime \prime} \mathrm{W}$ \\
Stony & Ontario & Marsh & $45^{\circ} 17^{\prime} 33.03^{\prime \prime N}$ & $75^{\circ} 48^{\prime} 25.29^{\prime \prime} \mathrm{W}$ \\
Jack & Ontario & Marsh & $44^{\circ} 32^{\prime} 1.82^{\prime \prime} \mathrm{N}$ & $76^{\circ} 22^{\prime} 35.31^{\prime \prime} \mathrm{W}$ \\
Barb & Ontario & Marsh & $44^{\circ} 31^{\prime} 27.54^{\prime \prime} \mathrm{N}$ & $76^{\circ} 22^{\prime} 25.89^{\prime \prime} \mathrm{W}$ \\
Osprey & Ontario & Marsh & $44^{\circ} 30^{\prime} 25.73^{\prime \prime} \mathrm{N}$ & $76^{\circ} 24^{\prime} 38.08^{\prime \prime} \mathrm{W}$ \\
\hline
\end{tabular}




\section{Host sampling}

Damselflies were collected weekly. At each site, host species assemblages were assessed, by collecting individuals for 20 minutes. Collecting all damselflies within an allotted time also allowed us to index each damselflies species' relative local abundance. After the initial 20 minute collecting period, sampling continued for up to three hours or until 30 individuals of each species at each site were collected. All the damselflies were stored in 95\% ethanol in individual vials. In the lab, damselflies were re-identified and examined for water mites.

\section{Parasite sampling and measures of parasitism}

Damselflies were examined for water mites using a ZEISS SteREO Discovery V8 dissecting microscope, all mites were quantified, collected and stored in $95 \%$ ethanol. I used (Rózsa et al. 2000) definitions of prevalence and mean intensity. I measured the number of Arrenurus 'species' hereafter Operational Taxonomic Units (OTUs) per host species, using a molecular dataset. For most of the parameters, I pooled the data from all the sites because there were no significant differences in prevalence $(\mathrm{F}=0.95 ; \mathrm{df}=12,80$; $\mathrm{p}=0.50)$ or intensity $(\mathrm{F}=0.97 ; \mathrm{df}=12,80 ; \mathrm{p}=0.48)$ across sites. Analyses were performed in QP3.0 (Rózsa et al. 2000).

\section{Composite host phylogeny construction}

To examine potential importance of a host species' evolutionary history to its likelihood of being parasitized by specific OTUs of water mites, I created a hypothesized phylogeny of Coenagrionidae collected in the marshes and bogs of Southeastern Ontario and 
Southwestern Quebec, by compiling recent phylogenies (Chippindale et al. 1999, Brown et al. 2000, May 2002, O'Grady and May 2003, Dumont et al. 2010). This approach allowed us to control for evolution of the characters among host species. Because molecular phylogenetic information is lacking for many of the damselfly species collected, taxonomy is a reasonable substitute to phylogeny (Crozier et al. 2005). I assumed that genera are monophyletic. I performed analyses of phylogenetic community structure and relatedness within each site species assemblage by assigning the age of the node splits using BLADJ (Branch Length Adjuster) module of Phylocom software package (Webb 2000). BLADJ places internal nodes where age estimates from phylogenetic trees were known. Age estimates were determined through the literature and discussion with experts. BLADJ also sets the remaining branch lengths by spacing the undated nodes in the tree evenly between those dated nodes. There are assumptions made, such as the true age of the splits but it reduces variance in branch lengths and the nodal distances are based on evolutionary time and are independent of species richness and levels of resolution within clades.

\section{Phylogeny of water mites}

To address whether host species of sibling species pairs might share mite OTUs, and to have a preliminary account of host species range of those mite OTUs, a subset of water mites were sent to the Barcode of Life Database, BOLD; www.boldsystems.org (Ratnasingham and Hebert 2007) for barcoding using Cytochrome Oxidase I (COI). Randomly chosen mites were sent to the Canadian Centre for DNA Barcoding (CCDB; www.ccdb.ca) at the Biodiversity Institute of Ontario to perform DNA barcoding using 
standard, high throughput methods (Ivanova et al. 2006). DNA extractions from 285 larval mites were performed. These larval water mites were selected randomly, based principally on host species and site of host collection. This random selection was as follows: if present, ten mites (five from the thorax and five from the abdomen because thoracic and abdominal mites are often different species (B. Smith, pers. comm.)), from every host species at every site were collected. Each mite was haphazardly chosen from a different host individual if there were enough infected individuals. Twelve of sixteen host species had thoracic mites, and eight of sixteen species also had abdominal mites. In those latter species, both thoracic and abdominal water mites were collected, which is why sample size was initially 285 . Chromatograms were edited and contiguous sequences were assembled using Sequencher v4.7 (Gene Codes Corp., Ann Arbor, MI, USA). COI sequences were aligned manually in Mesquite v2.74 (Maddison and Maddison 2011) according to the translated amino acid sequence. Sequences have been submitted to GenBank (KJ709142-KJ709343). Homologous sequences from four Arrenurus sp. individuals collected from Nehalennia gracilis and $N$. irene damselflies (Mlynarek et al. 2014) and four Enallagma ebrium (Mlynarek et al. 2013) were included in the COI alignment, and seven water mite species were selected from GenBank to serve as outgroups (AB530314， JX838402，JX836526， JX835088， JN018105， JN018109, AB530311).

Pairwise distances were calculated using neighbour-joining analysis with the uncorrected ("p") model in PAUP* v4.0b10 (Swofford 2003). Phylogenetic analysis of the COI dataset was performed using Bayesian inference (BI) in MrBayes v3.1.2 
(Huelsenbeck and Ronquist 2001,Ronquist and Huelsenbeck 2003). The best-fit model of molecular evolution was determined to be GTR $+\mathrm{I}+\mathrm{G}$, using MrModeltest v2.3 (Nylander 2004). Bayesian analysis was performed in MrBayes with a Markov Chain Monte Carlo (MCMC) method, two independent runs, with nucmodel $=4 \mathrm{by} 4, \mathrm{~N}_{\mathrm{st}}=6$, rates $=$ invgamma, samplefreq $=1000$, four chains $=$ one cold and three heated, 10 million generations. Excluding a $10 \%$ burn-in, the remaining trees were used to generate a majority-rule consensus tree.

\section{Host parameters}

I chose five host species' characteristics that have been reasonably strong explanatory variables of other host-parasite associations (Poulin 2007, Krasnov et al. 2004):

1- Geographic range size (Range) was assessed using the Odonata Central database (Abbott 2007). The records in this database are verified and managed regularly by experts. To determine the size of a host's geographic range, a $2^{\circ}$ latitude-longitude grid was superimposed on the geographic data. Presence-absence was then observed for each cell. The area of the species range was determined by adding the area of each cell where that species was present (see Hassall 2012 for details).

2- Regional representation (RR) of a host species was determined simply as the number of sites a host species was collected at within my sampling season. Since I collected at 13 sites, the maximum regional representation a species can have is 13 . Stated otherwise, if the regional representation of a host species is 13 , that host was collected at all the sites (if 12 , at 12 of 13 potential sites, etc.). 
3- I assessed relative local abundance by collecting damselflies at a site for 20 minutes. This was then labelled as the species assemblage for that site. From this, I could gauge how rich the host species assemblage was at a given site and the abundance of each host species there. Local abundance for my study is the proportion of a particular host species over the number of host species collected within 20 minutes. Because I collected at each site eight times, the relative local abundance is the average local abundance at each site for eight weeks. My final measure used for analysis is the mean local abundance (MLA). This is the grand mean of all the relative local abundances from all the sites.

4- I assessed phenology of a host by determining the week where $50 \%$ of the population of a species was collected using the accumulation curves (Fig 4.2). I determined peak of damselfly activity by calculating the mean of all the weeks where $50 \%$ of the population of a species. I then took, for each host species, the absolute value of the difference between the $50 \%$ week and the mean of all the weeks. Through this, I could assess whether the peak flight season of a host species was near the peak of damselfly activity across all species.

5- Using digital calipers, I measured wing length from the base to the tip of the right forewing, as a proxy for host body size. In this case, my measure of wing length is the mean of wing length from each damselfly species from all the sites.

\section{Statistics}

I evaluated the data using phylogenetically generalized least squares (PGLS), which was implemented using the package caper in R (Orme et al. 2012) . Fixed effect covariates 
included size of host range (Range), regional representation (RR), phenology (Phenology), Grand mean local abundance (MLA) and wing length (WingL). Model selection was based on Akaike's information criterion corrected for sample size (AICc) (Burnham et al. 2002). Regression coefficients (i.e. $\beta$-values on a logit scale) were estimated by model averaging and statistical significance was judged on the basis of sign (positive or negative relationship between the predictor and response variables) and the precision of estimates (wherein values with 95\% confidence intervals that did not overlap with zero were considered important predictors). My candidate set of models included all additive combinations of the predictor variables. To avoid over-parametrization, I did not evaluate interactions between variables.

\section{RESULTS}

Measures of general Arrenurus parasitism

A total of 7107 damselflies were collected (Table 4.2). Host abundance per species per site per week ranged from 61 damselfly individuals collected in Osprey Marsh to 219 host individuals collected in Stony Swamp. There was variation in the cumulative date of emergence of the damselfly species across the eight weeks (Fig. 4.2). There was also a lot of variation of Arrenurus prevalence and intensity between the host species (Fig. 4.3). Prevalence of parasitism varied between 0\% in Amphiagrion saucium (Burmeister), Chromagrion conditum (Selys), Coenagrion interrogatum (Selys) and Enallagma geminatum Kellicott to $37.4 \%$ in Enallagma ebrium (Table 4.2). For the infected species, mean intensity varied between 1.3 Arrenurus water mites in Enallagma carunculatum and 13.3 in Ischnura verticalis (Table 4.2). 

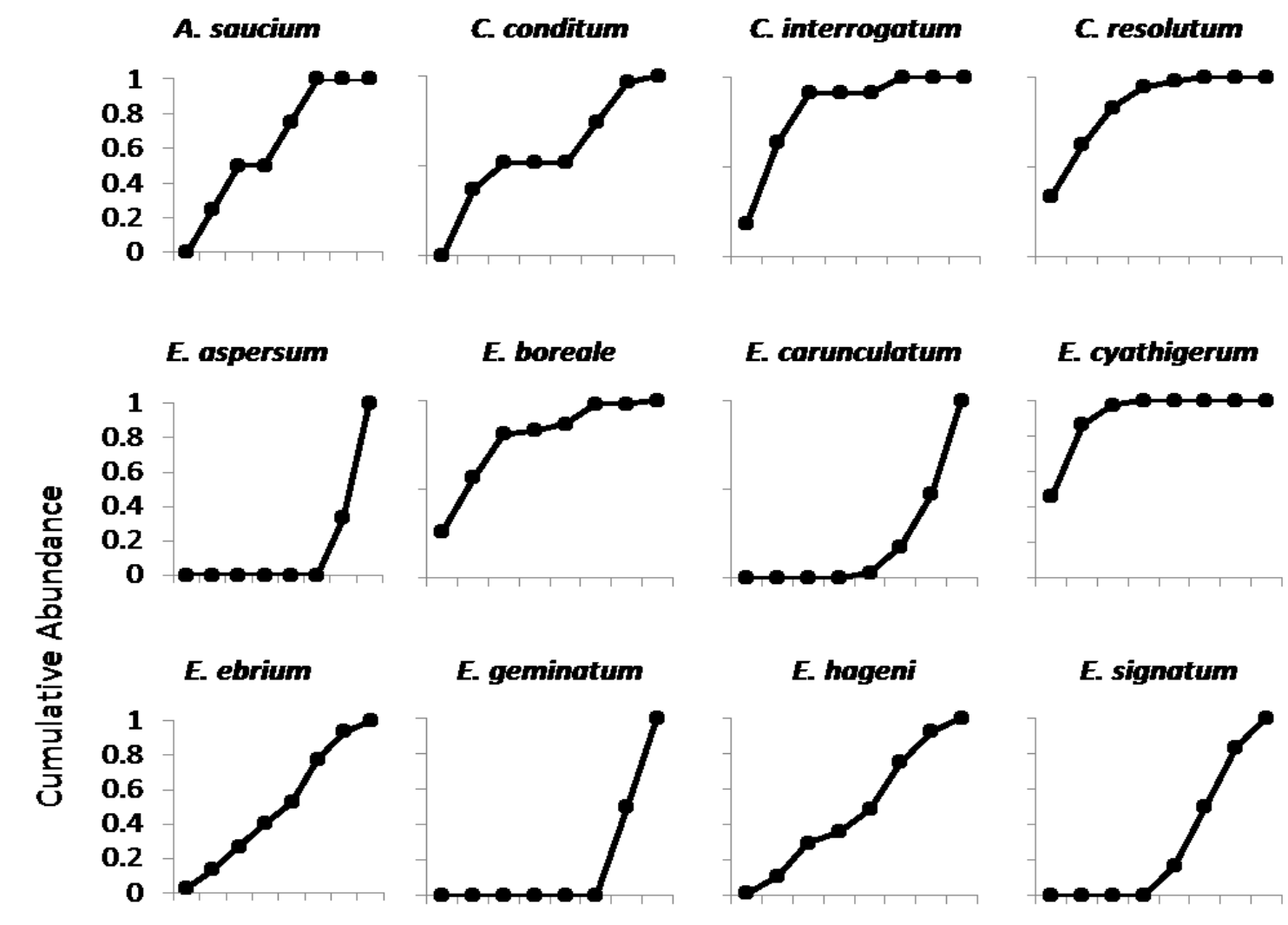

E. boreale

E. corunculotum
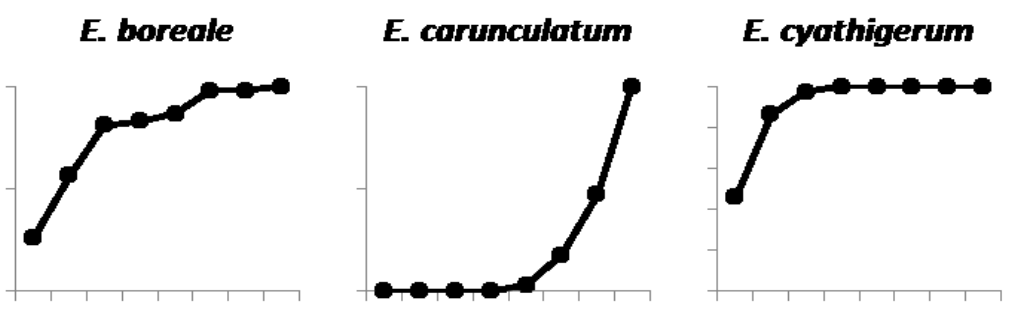

I. posito
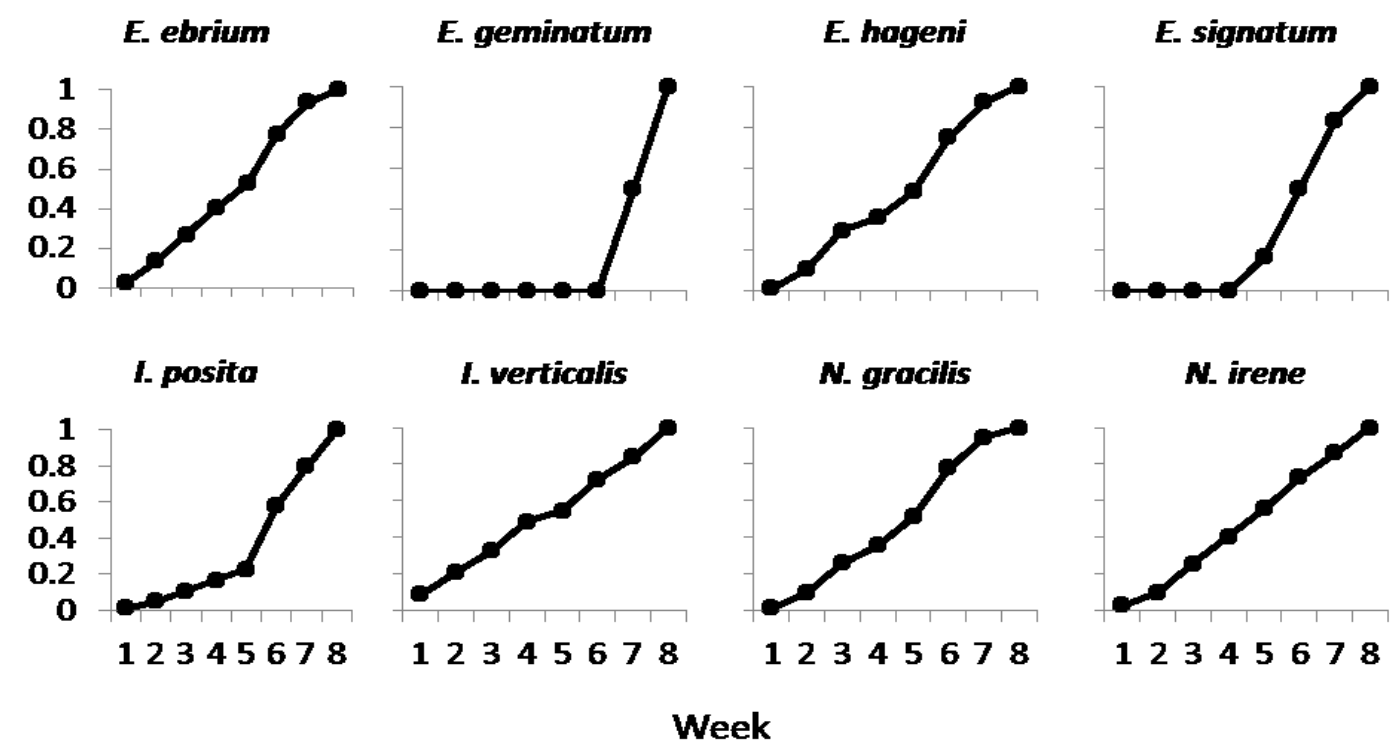

Week

Fig. 4.2. Abundance accumulation curves for the 2011 collecting season of the 16 damselfly species collected from 13 sites in Southeaster Ontario and Southeastern Quebec. 

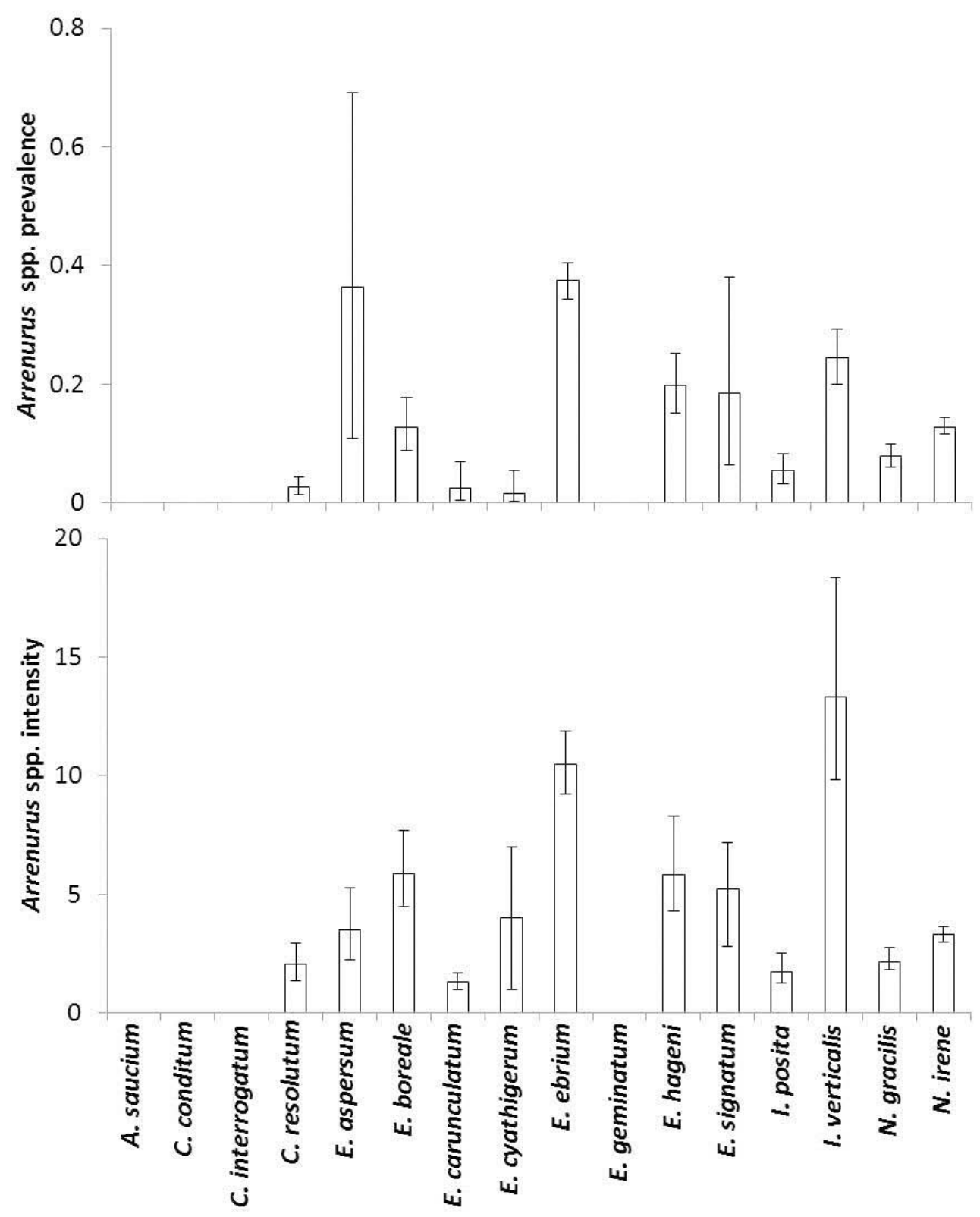

Fig. 4.3. Interspecific variation in (a) prevalence and (b) intensity of Arrenurus spp parasitism for the 2011 collecting season of the 16 damselfly species collected from 13 sites in Southeaster Ontario and Southeastern Quebec. 
Table 4.2. Host parameters of the 16 damselfly species that were collected during the reproductive season of 2011 from 13 sites in Southeastern Ontario and Southwestern Quebec. $\mathrm{N}=$ sample size of each species, RLA = Relative local abundance, $\mathrm{RR}=$ Regional representation; OTUs $=$ number of Operational Taxonomic units; WingL = wing length.

\begin{tabular}{|c|c|c|c|c|c|c|c|c|c|}
\hline Host Species & $\begin{array}{c}\mathrm{N} \\
\text { ( } \mathrm{N} \text { inf.) }\end{array}$ & $\begin{array}{c}\text { Prevalence } \\
(\%)\end{array}$ & Intensity & $\begin{array}{c}\text { Phenology } \\
|\mathrm{d}|\end{array}$ & $\begin{array}{c}\text { Range } \\
\left(10^{6} \mathrm{~km}^{2}\right)\end{array}$ & RLA & RR & WingL & $\overline{T U s}$ \\
\hline A. saucium & $\begin{array}{c}8 \\
(0)\end{array}$ & $\begin{array}{c}0.0 \\
(0-36.9)\end{array}$ & $\begin{array}{c}0.0 \\
\text { (NA) }\end{array}$ & 0.44 & 1.81 & 0.050 & 1 & 16.03 & 0 \\
\hline C. conditum & $\begin{array}{l}133 \\
(0)\end{array}$ & $\begin{array}{c}0.0 \\
(0-2.7)\end{array}$ & $\begin{array}{c}0.0 \\
(\mathrm{NA})\end{array}$ & 1.44 & 2.21 & 0.098 & 2 & 21.62 & 0 \\
\hline C. interrogatum & $\begin{array}{l}52 \\
(0)\end{array}$ & $\begin{array}{c}0.0 \\
(0-6.8)\end{array}$ & $\begin{array}{c}0.0 \\
\text { (NA) }\end{array}$ & 2.44 & 2.35 & 0.058 & 1 & 18.90 & 0 \\
\hline C. resolutum & $\begin{array}{l}542 \\
(15)\end{array}$ & $\begin{array}{c}2.6 \\
(1.4-4.3)\end{array}$ & $\begin{array}{c}2.1 \\
(1.4-2.9)\end{array}$ & 2.44 & 6.13 & 0.046 & 11 & 18.21 & 1 \\
\hline E. aspersum & $\begin{array}{l}11 \\
(4)\end{array}$ & $\begin{array}{c}36.4 \\
(10.9-69.2)\end{array}$ & $\begin{array}{c}3.5 \\
(2.3-5.3)\end{array}$ & 1.56 & 2.58 & 0.057 & 1 & 19.87 & 3 \\
\hline E. boreale & $\begin{array}{l}242 \\
(32)\end{array}$ & $\begin{array}{c}12.8 \\
(8.9-17.7)\end{array}$ & $\begin{array}{c}5.9 \\
(4.5-7.7)\end{array}$ & 2.44 & 6.63 & 0.046 & 6 & 20.00 & 3 \\
\hline E. carunculatum & $\begin{array}{l}123 \\
(3)\end{array}$ & $\begin{array}{c}2.4 \\
(0.5-7.0)\end{array}$ & $\begin{array}{c}1.3 \\
(1-1.7)\end{array}$ & 2.56 & 5.20 & 0.102 & 3 & 20.56 & $?$ \\
\hline E. cyathigerum & $\begin{array}{l}128 \\
(2)\end{array}$ & $\begin{array}{c}1.6 \\
(0.2-5.5)\end{array}$ & $\begin{array}{c}3.0 \\
(1-4)\end{array}$ & 3.44 & 6.50 & 0.044 & 6 & 19.81 & 3 \\
\hline E. ebrium & $\begin{array}{l}1000 \\
(374)\end{array}$ & $\begin{array}{c}37.4 \\
(34.3-40.5)\end{array}$ & $\begin{array}{c}10.5 \\
(9.2-11.9)\end{array}$ & 0.56 & 4.35 & 0.141 & 12 & 18.09 & 7 \\
\hline E. geminatum & $\begin{array}{c}3 \\
(0)\end{array}$ & $\begin{array}{c}0.0 \\
(0-70.8)\end{array}$ & $\begin{array}{c}0.0 \\
\text { (NA) }\end{array}$ & 2.56 & 2.98 & 0.047 & 1 & 18.48 & 0 \\
\hline E. hageni & $\begin{array}{l}257 \\
(51)\end{array}$ & $\begin{array}{c}19.8 \\
(15.1-25.3)\end{array}$ & $\begin{array}{c}5.8 \\
(4.3-8.3)\end{array}$ & 0.56 & 4.25 & 0.059 & 8 & 17.81 & 4 \\
\hline E. signatum & $\begin{array}{l}27 \\
(5)\end{array}$ & $\begin{array}{c}18.5 \\
(6.3-38.1)\end{array}$ & $\begin{array}{c}5.2 \\
(2.8-7.2)\end{array}$ & 2.56 & 3.23 & 0.079 & 2 & 20.25 & 2 \\
\hline I. posita & $\begin{array}{l}336 \\
(18)\end{array}$ & $\begin{array}{c}5.4 \\
(3.2-8.3)\end{array}$ & $\begin{array}{c}1.7 \\
(1.3-2.5)\end{array}$ & 1.56 & 3.13 & 0.051 & 9 & 15.92 & 3 \\
\hline I. verticalis & $\begin{array}{l}349 \\
(85)\end{array}$ & $\begin{array}{c}24.4 \\
(19.9-29.2)\end{array}$ & $\begin{array}{c}13.3 \\
(9.9-18.4)\end{array}$ & 0.44 & 4.36 & 0.063 & 13 & 16.27 & 6 \\
\hline
\end{tabular}




\section{Table 4.2. Continued}

\begin{tabular}{|c|c|c|c|c|c|c|c|c|}
\hline Host Species & $\begin{array}{c}\mathrm{N} \\
\text { ( } \mathrm{N} \text { inf.) }\end{array}$ & $\begin{array}{c}\text { Prevalence } \\
(\%)\end{array}$ & Intensity & $\begin{array}{l}\text { Phenology } \\
\qquad|\mathrm{d}|\end{array}$ & $\begin{array}{c}\text { Range } \\
\left(10^{6} \mathrm{~km}^{2}\right)\end{array}$ & RLA RR & WingL & $\overline{T U s}$ \\
\hline N. gracilis & $\begin{array}{l}820 \\
(64)\end{array}$ & $\begin{array}{c}7.8 \\
(6.1-9.9)\end{array}$ & $\begin{array}{c}2.2 \\
(1.8-2.8)\end{array}$ & 0.56 & 1.51 & $0.257 \quad 5$ & 14.88 & 2 \\
\hline$N$. irene & $\begin{array}{l}3076 \\
(395)\end{array}$ & $\begin{array}{c}12.8 \\
(11.7-14.5)\end{array}$ & $\begin{array}{c}3.3 \\
(3.0-3.6)\end{array}$ & 0.56 & 4.03 & $0.486 \quad 13$ & 15.60 & 4 \\
\hline
\end{tabular}


Host Phylogeny

I collected 16 species across all sites which were included in my phylogeny of the Coenagrionidae in marshes and bogs of Southeastern Ontario and Southwestern Quebec (Fig. 4.4), of which four species (25\%) were collected only at one site and two species (12.5\%) occurred at all sites (Table 4.2). All subsequent statistical analyses were run using this composite phylogeny to control for a potential evolutionary signal.

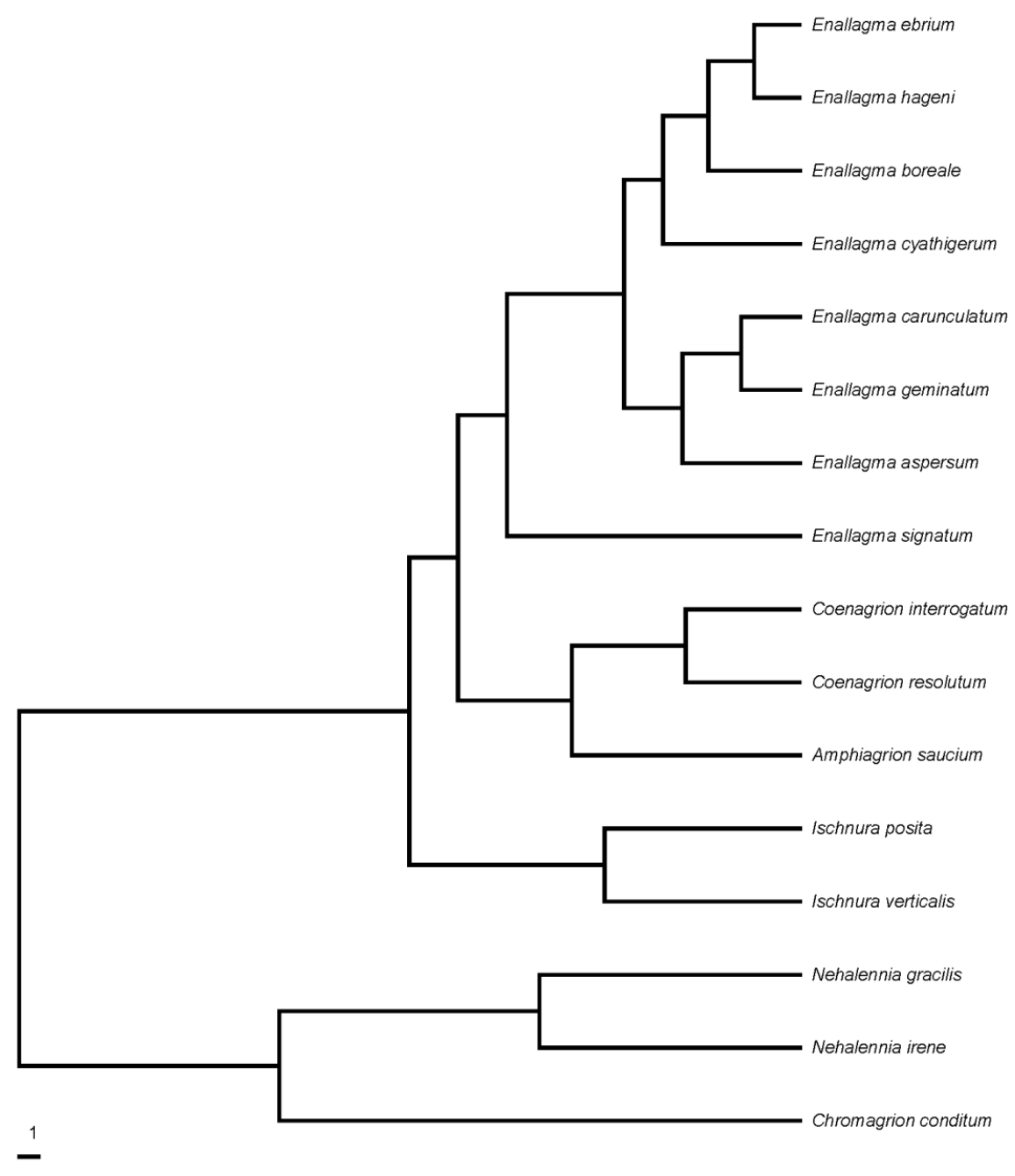

Figure 4.4. Composite phylogeny of the Coenagrionidae found at the thirteen sites of Southeastern Ontario and Southwestern Quebec. 
DNA barcoding

A 683bp fragment of COI was amplified from 217 Arrenurus specimens, a total of 327 characters were parsimony informative and 356 were uninformative.

Bayesian inference was performed on the COI dataset for 10 million generations. After burn-in there were 18002 trees, which were summarized in a 50\% majority rule consensus tree $(\mathrm{TL}=1370, \mathrm{CI}=0.396, \mathrm{RI}=0.927)$. This tree was well supported, with most nodes having moderate to high posterior probabilities (Fig. 4.5). The ingroup was divided into 15 well-supported clades, OTUs. Average interclade divergence was $10.7 \% \pm 5.8$, and average intraclade divergence was $0.7 \% \pm 0.5$. Average divergence between OTUs 1 and 2 was $16.6 \% \pm 0.6$, divergence between OTUs $3,4,5$ and 6 was $7.9 \% \pm 3.2$, between OTUs 8,9 , and 10 was $7.5 \% \pm 1.6$, and OTUs $11,12,13,14$ and 15 show $9.0 \% \pm 3.2$ divergence. Considering the high level of divergence between OTUs and the low divergence within each clade, as well as the strong support for each clade in the phylogenetic reconstruction, it appears that each OTU may be distinct. I are not implying they are new species and the possibility still exists that there are many more species present if speciation (and adaptive radiation) has occurred recently.

Based on the BI of water mites I determined that C. resolutum is infected by OTU 8. Enallagma aspersum (Hagen) by OTUs 9, 10 and 14, E. cyathigerum OTUs 7, 8 and 9. E. boreale gets OTUs 7, 8 and 10, E. ebrium by OTUs 4, 5, 8, 9, 10, 11 and 15, E. hageni (Walsh) has 4 OTUs 8, 9, 10 and 15, E. signatum has OTUs 4 and 12, I. posita has OTUs 8, 13 and 15 and $I$. verticalis has OTUs $5,8,11,13,14$ and $15 . N$. gracilis is infected by OTUs 1 and 2, N. irene is infected by OTUs 1, 3, 4 and 6. 


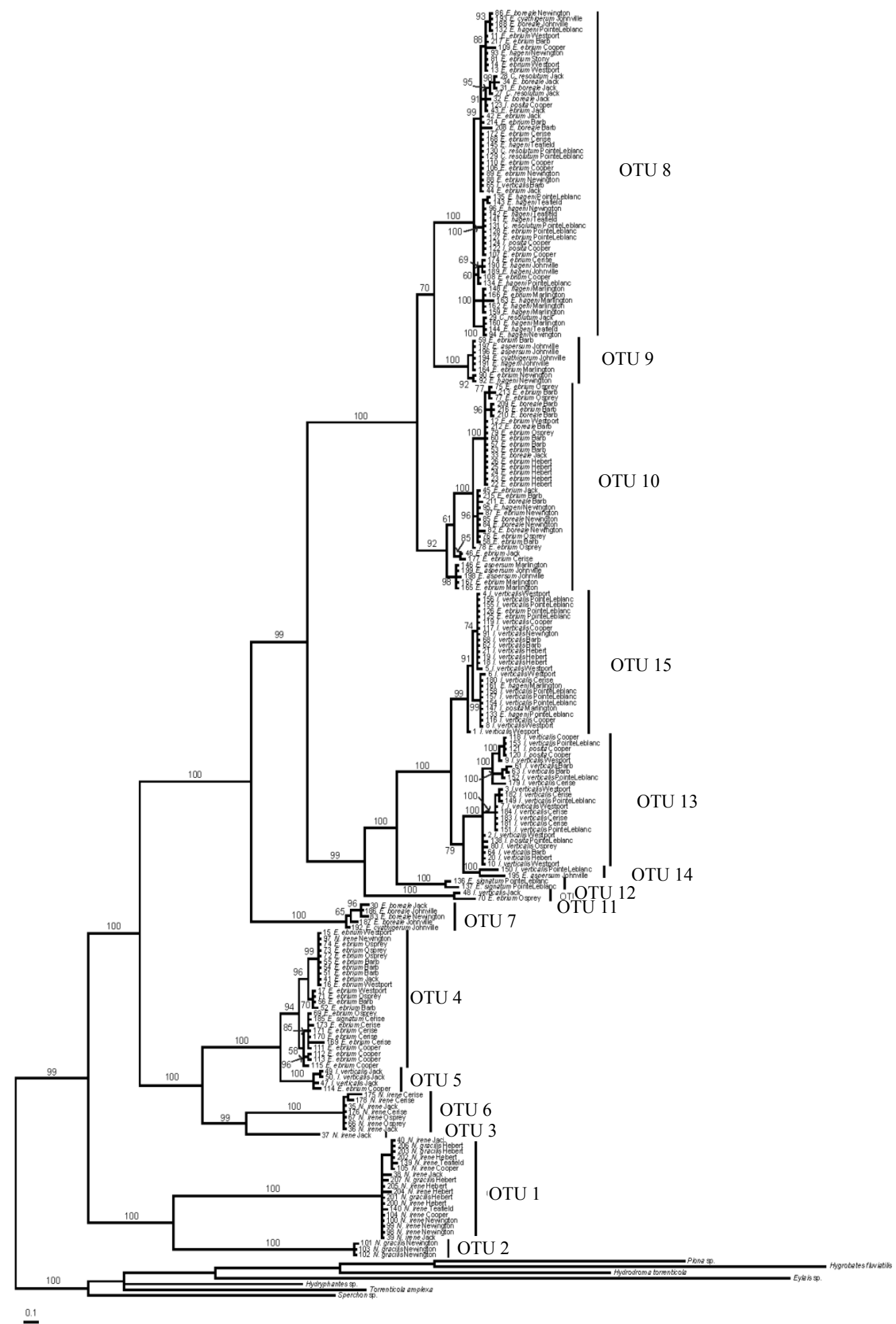

Figure 4.5. Majority rule consensus tree of 18002 trees generated by Bayesian MCMC analysis (10 million generations) of $683 \mathrm{bp}$ fragment of COI from 224 water mites, 217 ingroup and 7 outgroup specimens $(\mathrm{TL}=1370, \mathrm{CI}=0.40, \mathrm{RI}=0.93)$. 


\section{Statistics}

My PGLS examining variation in the prevalence of water mite parasitism in relation to host species characteristics indicated that Range and Phenology were important predictor variables $\left(\log (L)=-38.31, \mathrm{~K}=3, \mathrm{AIC} c=84.61, \omega_{\mathrm{i}}=0.37\right)$; several other models also were supported by the data $(\triangle \mathrm{AIC} c<4 ;$ Table 4.3). Model averaged regression coefficients (Table 4.4) showed a negative association between water mite prevalence and phenology $\left(\beta_{\text {Phenology }}=-10.10,95 \%\right.$ CI:-14.91 to -5.29 ; Fig $\left.4.6 \mathrm{a}\right)$ and a positive association with range ( $\beta_{\text {Range }}=2.75,95 \%$ CI: 0.82-4.68; Fig 4.6b). Host body size and local abundance had little predictive power (Table 4.4).

Table 4.3. Model selection results for PGLSs evaluating water mite prevalence in relation to host distribution attributes. Table includes the global model and all models with $\mathrm{AICc}<4$. Calculations are described in the footnote ${ }^{1}$.

\begin{tabular}{lccccccc}
\hline \multicolumn{1}{c}{ Model } & $\log (\mathrm{L})$ & $\mathrm{K}$ & $\mathrm{AIC} c$ & $\Delta \mathrm{i}$ & $\begin{array}{c}\text { Model } \\
\text { Likelihood }\end{array}$ & $\begin{array}{c}\text { Model } \\
\text { prob. }\end{array}$ & $\mathrm{R}^{2}$ \\
\hline Range+Phenology & -38.31 & 3 & 84.61 & 0.00 & 1.000 & 0.365 & 0.66 \\
Range+MLA+Phenology & -37.35 & 4 & 86.34 & 1.73 & 0.421 & 0.154 & 0.67 \\
RR+Phenology & -39.46 & 3 & 86.91 & 2.29 & 0.317 & 0.116 & 0.67 \\
Phenology & -41.15 & 2 & 87.22 & 2.61 & 0.271 & 0.099 & 0.60 \\
Range+Phenology+WingL & -38.05 & 4 & 87.74 & 3.13 & 0.209 & 0.076 & 0.63 \\
RR+Range+Phenology & -38.28 & 4 & 88.19 & 3.58 & 0.167 & 0.061 & 0.61 \\
RR+Phenology +WingL & -38.36 & 4 & 88.35 & 3.74 & 0.154 & 0.056 & 0.61 \\
RR+Range+MLA+Phenology & -37.22 & 6 & 103.24 & 17.62 & $<0.001$ & $<0.001$ & 0.57 \\
+ +WingL & \\
& \\
&
\end{tabular}


Table 4.4. Model averaged regression coefficients $(\beta)$ for Phylogenetic Generalized Least Squares (PGLSs) evaluating variation in water mite prevalence.

\begin{tabular}{ccccc}
\hline \multicolumn{5}{c}{ MODEL AVERAGED PARAMETERS } \\
\hline Variable & Estimate & $S E$ & $L C I$ & $U C I$ \\
\hline (Intercept) & 9.482 & 9.014 & -8.185 & 27.150 \\
RR & 0.218 & 0.170 & -0.115 & 0.552 \\
Range & 2.748 & 0.985 & 0.818 & 4.678 \\
MLA & -3.194 & 3.285 & -9.632 & 3.245 \\
Phenology & -10.101 & 2.455 & -14.913 & -5.289 \\
WingL & 0.179 & 0.219 & -0.250 & 0.609 \\
\hline
\end{tabular}
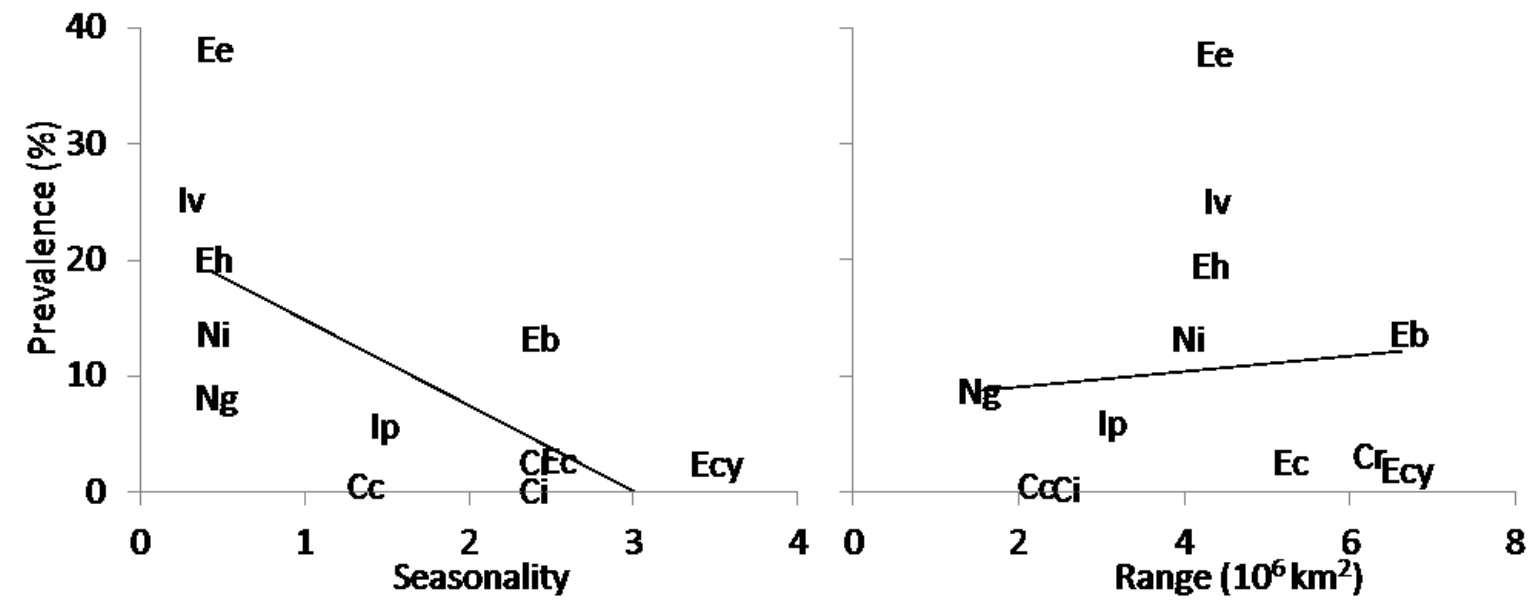

Figure 4.6. Summary of the important predicting factors explaining Arrenurus spp. prevalence. (a) Phenology and (b) range in relation to Arrenurus spp. prevalence. $\mathrm{Cc}=$ Chromagrion conditum $; \mathrm{Ci}=$ Coenagrion interrogatum $; \mathrm{Cr}=$ C. resolutum $; \mathrm{Eb}=$ Enallgma boreale $; \mathrm{Ec}=$ E. carunculatum $; \mathrm{Ecy}=$ E. cyathigerum $; \mathrm{Ee}=$ E. ebrium $; \mathrm{Eh}=$ E. hageni; $\mathrm{Ip}=$ Ischnura posita $; \mathrm{Iv}=I$. verticalis, $\mathrm{Ng}=$ Nehalennia gracilis $; \mathrm{Ni}=N$. irene. 
The best fitting model in my candidate set of PGLS investigating water mite intensity indicated that again range and phenology were significant predictor variables $\left(\log (L)=-27.35, \mathrm{~K}=3, \mathrm{AIC} c=63.71, \omega_{\mathrm{i}}=0.31\right)$. Additionally, other models were supported by the data (AICc $<4$; Table 4.5). Model averaged regression coefficients (Table 4.6) indicated a negative association with phenology $\left(\beta_{\text {Phenology }}=-2.46,95 \%\right.$ CI:3.81 to -1.11 ; Fig $4.7 \mathrm{a})$ and a positive association with range $\left(\beta_{\text {Range }}=1.05,95 \% \mathrm{CI}\right.$ : 0.39-1.71; Fig 4.7b). Host body size and local abundance had little predictive power (Table 4.6).

Table 4.5. Model selection results for PGLSs evaluating water mite mean intensity in relation to host distribution attributes. Table includes the global model and all models with AICc $<4$. Calculations are described in the footnote of Table S1.

\begin{tabular}{lccccccc}
\hline Model & $\log (\mathrm{L})$ & $\mathrm{K}$ & $\mathrm{AIC} c$ & $\Delta \mathrm{i}$ & $\begin{array}{c}\text { Model } \\
\text { Likelihood }\end{array}$ & $\begin{array}{c}\text { Model } \\
\text { Prob. }\end{array}$ & $\mathrm{R}^{2}$ \\
\hline Range+Phenology & -27.35 & 3 & 63.71 & 0.000 & 1.000 & 0.307 & 0.56 \\
Range+MLA+Phenology & -25.21 & 4 & 64.14 & 0436 & 0.804 & 0.247 & 0.66 \\
RR & -29.90 & 2 & 65.13 & 1.417 & 0.492 & 0.151 & 0.40 \\
RR+MLA & -28.89 & 3 & 66.78 & 3.068 & 0.216 & 0.066 & 0.44 \\
RR+Phenology & -29.30 & 3 & 67.60 & 3.889 & 0.143 & 0.044 & 0.45 \\
RR+Range+MLA+Phenology+ & -25.07 & 6 & 78.93 & 15.23 & $<0.001$ & $<0.001$ & 0.55 \\
WingL & & & & & & & \\
\hline
\end{tabular}


Table 4.6. Model averaged regression coefficients $(\beta)$ for Phylogenetic Generalized Least Squares (PGLSs) evaluating variation in water mite intensity.

\begin{tabular}{lcccc}
\hline \multicolumn{5}{l}{ MODEL AVERAGED PARAMETERS } \\
\hline Variable & Estimate & SE & LCI & UCI \\
\hline (Intercept) & 2.006 & 2.532 & -2.957 & 6.969 \\
RR & 0.215 & 0.092 & 0.034 & 0.397 \\
Range & 1.051 & 0.337 & 0.390 & 1.711 \\
MLA & -4.232 & 2.433 & -9.000 & 0.537 \\
Phenology & -2.456 & 0.689 & -3.807 & -1.106 \\
WingL & 0.012 & 0.018 & -0.024 & 0.048 \\
\hline
\end{tabular}
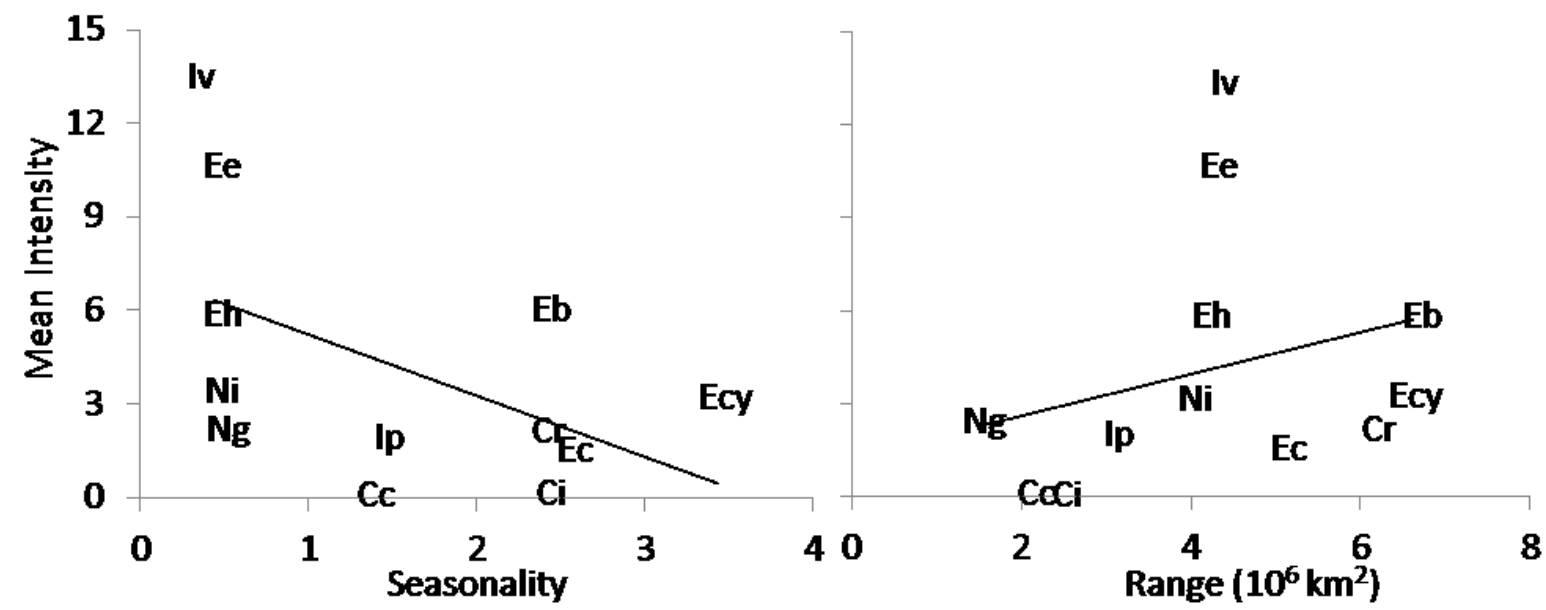

Figure 4.7. Summary of the important predicting factors explaining Arrenurus spp. mean intensity. (a) Phenology and (b) range in relation to Arrenurus spp. relationships. Cc $=$ Chromagrion conditum $; \mathrm{Ci}=$ Coenagrion interrogatum $; \mathrm{Cr}=$ C. resolutum $; \mathrm{Eb}=$ Enallgma boreale $; \mathrm{Ec}=$ E. carunculatum $; \mathrm{Ecy}=$ E. cyathigerum $; \mathrm{Ee}=$ E. ebrium $; \mathrm{Eh}=$ E. hageni; $\mathrm{Ip}=$ Ischnura posita $; \mathrm{Iv}=I$. verticalis, $\mathrm{Ng}=$ Nehalennia gracilis; $\mathrm{Ni}=N$. irene. 
My PGLS examining OTU richness in host species in relation to host species characteristics indicated that regional representation as the only significant predictor variable $\left(\log (L)=-27.29, \mathrm{~K}=2, \operatorname{AIC} c=59.71, \omega_{\mathrm{i}}=0.29\right)$; several other models also were supported by the data $(\triangle \mathrm{AIC} c<4$; Table 4.7). Model averaged regression coefficients (Table 4.8) indicated a positive association between regional representation and OTU richness $\left(\beta_{\mathrm{RR}}=0.362,95 \% \mathrm{CI}: 0.19\right.$ to 0.53 ; Fig 4.8$)$. Host body size and local abundance had little predictive power (Table 4.8).

Table 4.7. Model selection results for PGLSs evaluating water mite species richness (number of OTUs per host species) in relation to host distribution attributes. Table includes the global model and all models with $\mathrm{AICc}<4$. Calculations are described in the footnote of Table A1.

\begin{tabular}{lccccccc}
\hline Model & Log(L) & $\mathrm{K}$ & $\mathrm{AIC} c$ & $\Delta \mathrm{i}$ & $\begin{array}{c}\text { Model } \\
\text { Likelihood }\end{array}$ & $\begin{array}{c}\text { Model } \\
\text { prob. }\end{array}$ & $\mathrm{R}^{2}$ \\
\hline RR & & & & & & \\
RR+Phenology & -27.39 & 2 & 59.71 & 0.000 & 1.000 & 0.291 & 0.55 \\
RR+Range & -26.19 & 3 & 60.38 & 0.671 & 0.715 & 0.208 & 0.61 \\
RR+Phenology+WingL & -26.60 & 3 & 61.19 & 1.482 & 0.477 & 0.139 & 0.59 \\
RR+WingL & -25.23 & 4 & 62.09 & 2.378 & 0.304 & 0.089 & 0.62 \\
RR+MLA & -27.23 & 3 & 62.46 & 2.755 & 0.252 & 0.073 & 0.52 \\
RR+Range+WingL & -27.30 & 3 & 62.61 & 2.897 & 0.235 & 0.068 & 0.52 \\
RR+Range+MLA+Phenology+ & -25.61 & 4 & 62.85 & 3.138 & 0.208 & 0.061 & 0.63 \\
WingL & -24.60 & 6 & 70.54 & 10.831 & 0.004 & 0.001 & 0.60 \\
& & & & & & & \\
\hline
\end{tabular}


Table 4.8. Model averaged regression coefficients $(\beta)$ for Phylogenetic Generalized Least Squares (PGLSs) evaluating variation in water mite OTUs

\begin{tabular}{lcccc}
\hline \multicolumn{5}{l}{ MODEL AVERAGED PARAMETERS } \\
\hline Variable & Estimate & $S E$ & $L C I$ & $U C I$ \\
\hline (Intercept) & -0.276 & 1.670 & -3.550 & 2.998 \\
RR & 0.362 & 0.087 & 0.191 & 0.532 \\
Range & -0.100 & 0.063 & -0.224 & 0.024 \\
MLA & -0.086 & 0.405 & -0.881 & 0.708 \\
Phenology & -0.243 & 0.143 & -0.522 & 0.037 \\
WingL & 0.058 & 0.053 & -0.046 & 0.161 \\
\hline
\end{tabular}

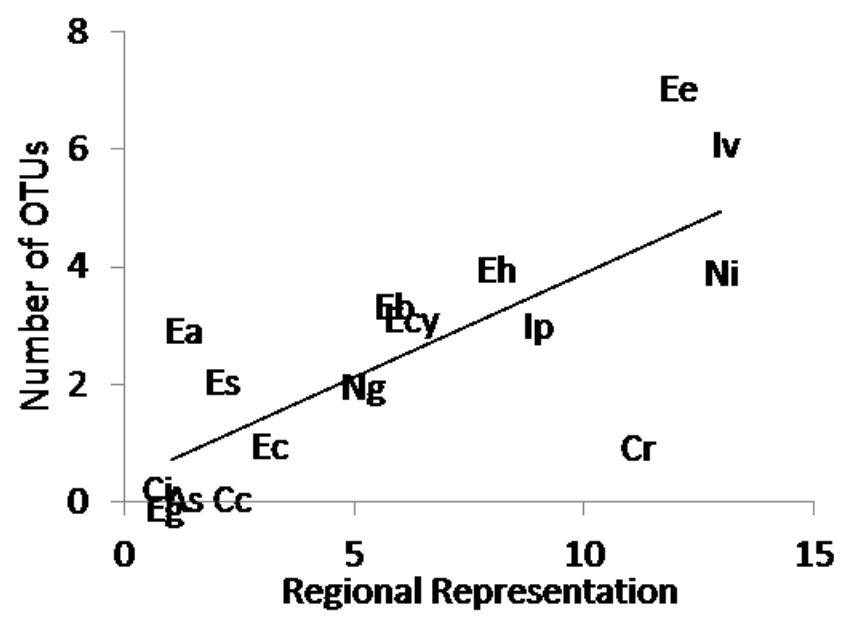

Figure 4.8. Summary of the important predicting factor explaining number of OTUs in each damselfly species. Host regional representation in relation to Arrenurus OTUs. As $=$ Amphiagrion saucium $; \mathrm{Cc}=$ Chromagrion conditum $; \mathrm{Ci}=$ Coenagrion interrogatum $; \mathrm{Cr}$ $=C$. resolutum $; \mathrm{Ea}=$ Enallgma aspersum $; \mathrm{Eb}=$ E. boreale $; \mathrm{Ec}=$ E. carunculatum $;$ Ecy $=$ E. cyathigerum; $\mathrm{Ee}=$ E. ebrium $; \mathrm{Eg}=$ Enallagma geminatum $; \mathrm{Eh}=$ E. hageni $; \mathrm{Es}=$ Enallagma signatum; $\mathrm{Ip}=$ Ischnura posita $; \mathrm{Iv}=I$. verticalis, $\mathrm{Ng}=$ Nehalennia gracilis; $\mathrm{Ni}=N$. irene. 


\section{DISCUSSION}

The goal of this study was to determine what host species' characteristics predict the levels of parasitism (prevalence and intensity) and the diversity of parasite interactions. Based on my results, it is clear that host distribution characteristics can predict damselflywater mite associations. Differences in prevalence and intensity of Arrenurus were explained mostly by two host characteristics, range and phenology, as measured in this study. The differences in number of OTUs can be predicted by a hosts' regional representation.

Host geographic range and phenology are important in predicting prevalence and intensity. A positive relationship between size of host geographic range and the prevalence and intensity of parasitism has previous been reported. Price et al. (1988) proposed the geographic range hypothesis with the prediction that more widespread hosts will be under higher parasite pressure. Tella et al. (1999) supported this hypothesis in a study of diurnal birds of prey parasitized by hematozoan blood parasites where they proposed that the pattern was potentially due to the evolutionary history of specific hostparasite associations. Similarly, the prediction of the geographic range hypothesis was supported in a study comparing two species of Calopteryx in Finland (Ilvonen et al. 2011). In contrast, the geographic range hypothesis was not supported in two studies comparing gregarine and water mite parasitism in several pairs of closely related coenagrionid damselflies where the less widespread species of the species pairs had higher parasitism levels (Mlynarek et al. 2012, Mlynarek et al. 2013). 
In the current study based on an increased number of non-phylogenetically paired host species, I find support for the geographic range hypothesis. However, geographic range alone was not able to predict the prevalence and intensity of parasitism. Host phenology was a strong predictor of prevalence and intensity in Arrenurus spp. parasitism. In systems where hosts demonstrate cyclical life history traits, such as the yearly life cycle of many insects, infection levels are expected to vary with time within that cycle (Forbes et al. 2012). This has been often demonstrated in infectious diseases (Altizer et al 2006), bees infected by internal Crithidia parasites (Gillespie 2010), insects infected by gregarines (Zuk 1987, Locklin and Vodopich 2010). As previous studies (e.g. Gillespie 2010), a seasonal unimodal pattern of parasitism was demonstrated in infection levels. This seasonal pattern could be linked to host density where host density may be either the size of the population of one host species (Forbes et al. 2012) or the aggregate density of all hosts in the species assemblage (Gillespie 2010). There is the expectation that parasites time their life cycle to coincide with that of their hosts to increase their chances of finding a host; in this case, the generalist parasite will track when there is the highest density of potential hosts or the peak of the host flight season.

Even if generalist parasites can survive on many host species they demonstrate preferences in host use (Tripet and Richner 1997). In this study, generalist parasite species richness or number of OTUs each damselfly species was infected by, was not predicted by host range size or phenology but only by host regional representation. Host species present at more sites were infected by more OTUs. Durrer and Schmid-Hempel (1995) found similar results when comparing bumblebee species infected by all their 
parasite taxa. One explanation could be the fact that if a species is present at more sites it will encounter more parasite species. I propose that the influence of host regional representation may be explained by the fact that widespread hosts have not adapted species specific recognition at particular sites and resistance or evasion does not occur. If a host species is cosmopolitan there may be more genetic mixing between those species populations and they are more likely to be in an open population, which could maintain their susceptibility to parasitism (Forbes and Mlynarek 2014). Mlynarek et al. (2014) has documented this type of pattern in Nehalennia, where N. gracilis, a habitat specialist and a closed population species, resists all of an Arrenurus sp. whereas $N$. irene, a ubiquitous and potentially open population species, does not show any resistance to the same parasite at the same two sites.

The other two predictor variables tested had a minor effect on explaining any of the measures of parasitism observed in this study. Based on the literature, I expected host body size to be a good predictor variable. Studies in ectoparasites of rodents (Krasnov et al. 2004), parasites of fissiped carnivores (Lindenfors et al. 2007), and bat flies on bats (Patterson et al. 2008) clearly demonstrated that host size mattered. Even in the invertebrate-parasite literature, host size has been shown to be an important predictor in intraspecific studies (Forbes and Baker 1991). There are other studies that show the opposite; there was a lack of support for a relationship between ectoparasites infecting flying squirrels and host size (Perez-Orella and Schulte-Hostedde 2005). Similarly in my study, host size did not predict parasitism levels. The other factor that had little effect was host local abundance. This was also unexpected because a relationship between local 
abundance and parasite levels has been previously reported (Arneberg et al. 1998, Krasnov et al. 2002). I would expect that more locally abundant species would be stronger targets of selection by the parasites. Local adaptation is expected to evolve in the parasites of locally abundant hosts (Arneberg et al. 1998). Even though host abundance and host size were expected to predict host parasitism, in this system, the better predictors are ones that are measured at larger scales.

Even though hosts were collected at multiple sites within a region, I superimposed the parasitism over the entire range considering it a species' characteristic, which is common in evolutionary ecology studies (Hefferman et al. 2014). The importance of scale has long been understood to be critical in ecology (Wiens 1989, Levin 1992) but rarely has it been tested in empirical studies (Tack et al. 2014). Different measures of species' characteristics and species' interactions might depend on different geographic or temporal scales (Hefferman et al. 2014) thus underscoring the need of measuring host species' characteristics of different scales for a better understanding of the relationship between the host and parasite species assemblages.

In this study, I primarily looked at Coenagrionidae-Arrenuridae host-parasite associations from the perspective of host species to explain three measures of parasitism; prevalence, intensity and diversity. As in other host-parasite associations, host characteristics do matter (Poulin 2007). This study is one of the few in invertebrate host systems that elucidate the reasons, based on host characteristics measured at multiple scales, of the strength of host-parasite associations. Further research into host-parasite 
associations in the context of macrosystems ecology, including understanding turnover of principal host species and host ranges across space and time would be logical next steps in understanding the evolution of the associations between hosts and their parasites. 


\section{CHAPTER FIVE}

Constitutive measures of immunity do not explain resistance across Coenagrionidae (Zygoptera)-Arrenurus (Arrenuridae: Acari) associations. 


\section{ABSTRACT}

Related host species often demonstrate different levels of parasite resistance. The mechanisms underlying this interspecific variation in resistance expression are not yet well understood. In invertebrates, innate immunity protects hosts from pathogens and parasites. A commonly used measure of innate immunity levels is Phenoloxidase (PO) activity. PO forms a principal part of a cascade that kills parasites. As such, PO activity levels are expected to reflect natural parasite resistance. Few researchers have assessed relations between PO activity and the level of resistance to parasites seen in nature across multiple host species. The goal of this study was to determine whether interspecific variation in innate immunity predicted interspecific variation in resistance to larval Arrenurus water mites for ten closely related species of coenagrionid damselflies (grouped into five species pairs). Two of five pairs have different levels of resistance to larval water mites and another two of five species pairs show differences in PO activity. Species with the highest resistance within the two pairs did not have corresponding higher PO activity levels. Furthermore, species pairs with no difference in resistance sometimes differed in PO activity levels. We conclude that PO activity alone is not adequate to predict a species' relative ability to resist particular parasites. 


\section{INTRODUCTION}

Explaining interspecific variation in parasite burden, among related host species, has been one of the main goals of evolutionary parasitology (Poulin 2007). In some taxonomic groups, like damselflies, variation in parasitism can occur among related host species even when ecological variables such as habitat use are controlled for (Mlynarek et al. 2012, Mlynarek et al. 2014). In other associations, ecological and evolutionary factors can explain differences in parasitism between host species to some extent (Morand and Krasnov 2010), but unexplained variation often remains. Evaluating patterns of resistance to parasites might help understand interspecific variation to parasites. For example, studies with closely related odonate host species have documented differential resistance to parasites (Forbes et al. 1999, Nagel et al. 2011, Mlynarek et al. 2013, Rynkiewicz et al. 2013). It is hypothesized, but untested, that interspecific differences in resistance to parasites are mirrored simply by interspecific differences in investment in innate immunity mechanisms.

In invertebrates, innate immunity protects a host from invaders. A common defense mechanism is the encapsulation of foreign substances through the process of melanisation via the Phenoloxidase (PO) cascade (Soderhall and Cerenius 1998) which is well documented (Marmaras et al. 1996, Gonzalez-Santoyo et al. 2010, Sugumaran 2002, Moreno-Garcia et al. 2013, Kanost and Gorman 2008). However, the extent to which PO activity can accurately predict a hosts' ability to respond immunologically to naturally occurring parasites is not well understood. A review of 23 studies by Gonzalez et al. 
(2012) questioned the reliability of PO activity in terms of immune function. The insect immune system is complex and is composed of a number of components in addition to PO (Wilson and Cotter 2013).

Most studies in ecological immunology have focused on within-species variation which can help to test factors influencing levels of naturally occurring parasites. However, there is little knowledge of the differences in innate immunity between species. These interspecific investigations are less common because of the problem of controlling for variation in life history between species that might influence immunity (e.g., emergence periods which determine when some insects can be collected also affect degree of temperature related immune responses). Species that invest more in innate immunity might do so at the cost of other life history traits. Even though PO activity may not be a reliable measure at the within species level (Gonzalez et al. 2012), it may be important in understanding variation in parasitism, resistance and immunity between different host species. Notwithstanding, I predict that host species that resist parasites invest more in innate immunity than species with less or no resistance to the same (or similar) parasites. Exploring this relationship provides further evidence of whether resistance to macroparasites actually does parallel PO activity (or innate immunity) levels seen in host species. I expect that host species with high natural resistance to parasites should have high levels of constitutive innate immune response.

More specifically, the purpose of this study was to determine whether interspecific variation in a constitutive measure of innate immunity (PO activity) 
predicted interspecific variation in resistance in closely related species. In order to answer this question, I used damselfly-water mite associations. Damselflies are good model hosts to investigate innate immunity and host-parasite interactions for two main reasons (Yourth et al. 2002, Nagel et al. 2011). First, resistance to water mite parasitism can easily be assessed; the immunological response to water mite infection is melanotic encapsulation of the mite's feeding tube, the stylostome (Forbes et al. 1999). Larval water mites are killed by their hosts via melanisation, and often remain attached to the host as dead and unengorged larvae throughout the rest of the host's adult life (Forbes et al. 1999). This makes it possible to compare how related damselfly species respond to the same or similar species of mites (Forbes and Robb 2008). Second, studies have shown that different species have variable levels of mite parasitism and resistance (Mlynarek et al. 2012, Mlynarek et al. 2013). In the studies were variation in levels of parasitism was demonstrated, the host species were grouped into evolutionarily related species pairs that differed in their geographic range to test whether the size of the host's geographic range had an effect on parasitism (Mlynarek et al. 2012, Mlynarek et al. 2013). I also wanted to determine whether resistance and PO activity could be explained by host geographic range.

I had four specific objectives. First, I documented water mite parasitism and host resistance in ten species of coenagrionid damselflies (grouped into five species pairs to control for phylogenetic relatedness among host species but with differing geographic range). Second, I compared PO activity and Arrenurus spp. resistance within each species pair. Third, I determined whether host geographic range could explain any of the 
variation. Finally, I calculated whether the magnitude of differences in PO activity reflects the magnitude of differences in natural mite resistance. I therefore addressed interspecific variation in insect resistance and determined whether this variation can be predicted between related host species.

\section{METHODS}

\section{Host Collecting}

Using aerial sweep nets, I collected individuals from ten Coenagrionidae species, Argia moesta, Argia violacea, Enallagma boreale, Enallagma ebrium, Enallagma signatum, Enallagma vesperum, Ischnura posita, Ischnura verticalis, N. gracilis, and N. Irene, placed in five species pairs, Argia, Enallagma (Enallagma) subgenus, Enallagma (Chromatallagma) subgenus, Ischnura, and Nehalennia. The four Enallagma species are considered in two different species pairs, because the most current phylogeny for this species-rich genus places these species in two subgenera (May 2002). Past studies have used these species to discern interspecific variation in parasitism with internal and external parasites (Mlynarek et al. 2012, Mlynarek et al. 2013). Species pairs were collected at different sites, but both species within a species pair were collected at the same site and time to exclude potential diurnal or seasonal effects. The Nehalennia species pair was collected at Hebert Bog $\left(44^{\circ} 29^{\prime} 54.69^{\prime \prime} \mathrm{N}, 7^{\circ} 24^{\prime} 53.66^{\prime \prime} \mathrm{W}\right)$ on 7 and 30 June 2011, and 24 June 2012; E. (Chromatallagma) and Argia species pairs were collected at the edge of Lake Opinicon $\left(44^{\circ} 33^{\prime} 56.32^{\prime} \mathrm{N}, 76^{\circ} 19^{\prime} 26.46^{\prime} \mathrm{W}\right)$ on 30 June to 3 July, 2010 and from 3 to 10 July, 2010, respectively, and 17 July 2012; the species of the E. (Enallagma) clade were collected at Barb Marsh (44³1'27.54’N, 76²2’25.89’'W) on 
25 May 2010, from 7 to 10 June 2010 and on 20 June 2012; Ischnura species pair was

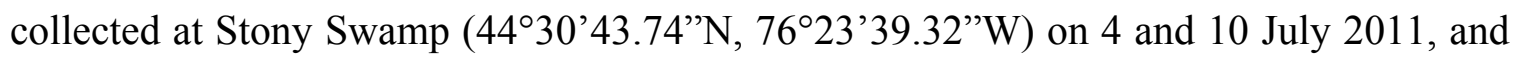
on 1 July 1012. 2010 or 2011 collections were used to assess host species differences in resistance to same or similar mite species, whereas 2012 collections were done to assess species similarities or differences in PO activity, within species pairs.

\section{Mite resistance}

Adult damselflies were collected during the reproductive seasons of 2010 and 2011. Damselflies were stored in separate vials containing 95\% ethanol before processing. Each damselfly was examined with a Zeiss SteREO Discovery.v8 dissecting microscope. Resisted water mites are recognized as flat, dark with no separation between the ventral sclerites or dorsal plate in comparison to live mites which are round, red or white (if preserved in ethanol) with ventral sclerites separated by non-scleritized tissue (Forbes et al. 1999). Numbers of live and resisted water mites were tallied. It is common for closely related host species collected from the same site to share the same water mite species (Mlynarek et al. 2013) so the potential is strong that the hosts are showing immune responses to the same parasites.

Innate immunity (Phenoloxidase (PO) activity)

Adult damselflies were collected, at the same sites as for the resistance analyses in the summer 2012. I collected only hosts that were not infected by water mites so as to not have to correct for amount for PO already mobilized to combat parasitism. There has been little variation in mite prevalence and resistance between years in these damselfly 
populations (unpubl data). The damselflies were stored in separate vials and placed into an ice-filled cooler until further processing at the lab (Iserbyt et al. 2012). They were stored overnight in a fridge at $3^{\circ} \mathrm{C}$. After removal from the fridge each individual was placed in liquid nitrogen for 10 seconds and placed in $-80^{\circ} \mathrm{C}$ freezer.

The protocol used for the PO assay is as in Stoks et al. (2006) and Iserbyt et al. (2012). In brief, the damselflies were dissected by removing the head, pronotum, wings, legs and abdomen from the excised thorax. The thorax, in an eppendorf tube, was dipped in liquid nitrogen for 10 seconds and crushed with a hand-held pestle. In order to extract the PO, $300 \mu \mathrm{l}$ of cooled cacodylate buffer $\left(0.01 \mathrm{M} \mathrm{C}_{2} \mathrm{H}_{6} \mathrm{AsNaO}_{2}-0.005 \mathrm{M} \mathrm{CaCl}_{2}\right)$ was added to each eppendorf tube containing the crushed thorax. The cell walls of the thorax were removed by centrifugation at $4^{\circ} \mathrm{C}$ at a speed of $15000 \mathrm{rpm}$ for 10 minutes. From each thoracic extract, $100 \mu 1$ was placed into a well of the 96 -well microplate along with $35 \mu 1$ PBS buffer, $5 \mu 1 \alpha$-chymotrypsin and was allowed to react for 5 minutes. After five minutes, $60 \mu \mathrm{l}$ of L-DOPA $(10 \mathrm{mmol} / \mathrm{L}$ in cacodylate buffer) was added followed by thorough mixing.

Next, the cooled microplate was placed in a spectrophotometer (FLUOstar OPTIMA microplate reader, BMG Labtech). The temperature sensitive reaction proceeded for $30 \mathrm{~min}$ at $30^{\circ} \mathrm{C}$ using an excitation filter of wavelength 485 . Twenty cycles were performed with $89 \mathrm{~s}$ duration per cycle. All absorbance readings were measured at the beginning of each cycle. Between two readings the microplate was shaken for 10s prior to the beginning of a new cycle. Spectral changes occur during this 30 -min period 
due to temperature sensitive nature of this enzymatic reaction. PO activity was scored as the slope of the absorbance-time regression during the linear phase of the reaction. The first five measurements were consistently disregarded because the enzymatic reaction, hence the regression fit, had not yet reached the appropriate linear phase.

As part of a correction for the PO assay, I measured and corrected for thoracic protein content. Total protein content (including the content of the PO enzyme) was expected to rise with individual body size. PO activity should therefore be controlled for total protein content (Stoks et al. 2006, Iserbyt et al. 2012). The protein content was measured as follows: $5 \mu 1$ of the thorax extract was placed in a new 96-well microplate with $155 \mu \mathrm{l}$ Mili-Q and $40 \mu \mathrm{l}$ Bradfort reagent. The microplate was placed in the spectrophotometer (FLUOstar OPTIMA microplate reader, BMG Labtech) using an absorption filter wavelength of 595 . The reaction was run at $30^{\circ} \mathrm{C}$ for 6 minutes with continuous shaking. Protein content was then determined by an endpoint reading and, within each plate, compared with a standard curve with known concentrations of Bovine serum albumin (United States Biochemical Corp, Bath - UK). Both physiological parameters (PO activity and protein content) were assayed twice to allow replication and to reduce measurement errors. The first and second measurements were typically strongly correlated for PO activity (Pearson correlation: $\mathrm{r}=0.99 ; \mathrm{N}=427 ; \mathrm{P}<0.001$ ) and for Protein content (Pearson correlation: $\mathrm{r}=0.89 ; \mathrm{N}=427 ; \mathrm{P}<0.001$; see also Iserbyt et al. 2012). The mean of both readings was used for further analyses. 


\section{Statistical analyses}

To control for phylogenetic effects, I performed analyses for prevalence of mite resistance and PO activity level within species pairs only (i.e. within the same genus or subgenus). However, I also tabulated results among species pairs more generally (e.g. whether those showing differences in resistance also show any differences in measures of innate immunity or not). Prevalence of resisted mites was defined, in this study, as the proportion of infected host individuals with at least one resisted water mite. I made the assumption that all the resisted mites were killed of melanisation (cf. Yourth et al. 2002) by the host because there was rarely high aggregation of parasites in the same region that would suggest competition between the parasites.

To test for differences between species of species pairs in prevalence of resisted mites, I ran five separate Fisher Exact two-tailed tests. I performed this test because the sample sizes for certain species pairs (i.e. Ischnura) were small (Zar 1999) and I wanted to keep the statistical analyses consistent for all the species pairs.

To test for differences in PO activity between species in the species pairs, I performed separate ANCOVAs for each of the five species pairs. In the ANCOVAs, PO activity was the response variable, species was the fixed variable and the covariate was thoracic protein content, to control for the size of host. I accounted for potential sex differences in PO activity (Iserbyt et al., 2012) by adding sex as a random variable.

To test whether PO activity is correlated with prevalence of resistance, I performed a Pearson correlation. I first corrected for individual and species differences in 
size and protein content by calculating the PO residuals obtained by regressing PO activity against thorax protein content. I then performed the Pearson correlation of the mean PO activity controlled by thoracic protein content versus mean prevalence of resistance of all ten species to determine whether estimates for innate immunity and parasite resistance co-vary across species. All the analyses were performed in JMP v.10.0.2 (SAS 2012).

To explore any potential effects of geographical range on parasite resistance and PO activity, I simply inspected whether any significant differences between species in species pairs seemed to be predicted reliably by either the more or less widely distributed host species having either the higher or lower prevalence or intensity of Arrenurus spp. infection.

\section{RESULTS}

\section{Mite resistance}

A total of 1451 damselflies were collected to tabulate natural resistance (Table 5.1). From the proportion of infected host individuals, prevalence of resistance ranged from no host individuals resisting parasites in $E$. $(E)$ boreale and $N$. irene to all host individuals resisting water mites in $N$. gracilis (Fig. 5.1a). 
Chapter 5

Table 5.1. Sample size (N) and number of hosts that were infected by Arrenurus spp., prevalence of resisted mites and PO activity in 10 species of damselflies, grouped into 5 species pairs based on phylogenetic relatedness.

\begin{tabular}{|c|c|c|c|c|c|c|}
\hline \multirow[t]{2}{*}{ Species Pair } & \multirow[t]{2}{*}{ Species } & \multirow{2}{*}{$\begin{array}{c}\text { Range } \\
\left(10^{6} \mathrm{Km}^{2}\right)\end{array}$} & \multicolumn{2}{|c|}{ Resistance } & \multicolumn{2}{|c|}{ PO activity } \\
\hline & & & $\begin{array}{c}\mathrm{N} \\
\text { (\# inf) }\end{array}$ & $\begin{array}{l}\text { Prevalence } \\
( \pm 95 \% \text { CI })\end{array}$ & $\mathrm{N}$ & $\begin{array}{c}\text { Values } \\
(\lambda / \min \pm \mathrm{SE})\end{array}$ \\
\hline \multirow[t]{4}{*}{ Argia } & A. moesta & 4.10 & 90 & 0.25 & 60 & 5.18 \\
\hline & & & (4) & $(0.01-0.81)$ & & $( \pm 0.25)$ \\
\hline & A. violaceae & 3.98 & 97 & 0.33 & 25 & 1.34 \\
\hline & & & (9) & $(0.07-0.70)$ & & $( \pm 0.16)$ \\
\hline \multirow[t]{4}{*}{ Enallagma $(E)$} & E. boreale & 6.53 & 99 & 0.00 & 40 & 2.61 \\
\hline & & & (23) & $(0.00-0.15)$ & & $( \pm 0.24)$ \\
\hline & E. ebrium & 4.35 & 311 & 0.13 & 30 & 2.56 \\
\hline & & & (104) & $(0.07-0.20)$ & & $( \pm 0.19)$ \\
\hline \multirow[t]{4}{*}{ Enallagma $(C)$} & E. signatum & 3.23 & 120 & 0.13 & 28 & 0.67 \\
\hline & & & (32) & $(0.04-0.29)$ & & $( \pm 0.06)$ \\
\hline & E. vesperum & 2.6 & 101 & 0.57 & 49 & 0.74 \\
\hline & & & (53) & $(0.42-0.70)$ & & $( \pm 0.06)$ \\
\hline \multirow[t]{4}{*}{ Ischnura } & I. posita & 3.13 & 124 & 0.10 & 27 & 1.18 \\
\hline & & & (10) & $(0.00-0.45)$ & & $( \pm 0.12)$ \\
\hline & I. verticalis & 4.36 & 17 & 0.50 & 59 & 2.37 \\
\hline & & & (2) & $(0.01-0.99)$ & & $( \pm 0.20)$ \\
\hline \multirow[t]{4}{*}{ Nehalennia } & N. gracilis & 1.51 & 208 & 1.00 & 49 & 0.51 \\
\hline & & & $(25)$ & $(0.86-1.00)$ & & $( \pm 0.04)$ \\
\hline & $N$. irene & 4.03 & 284 & 0.00 & 60 & 0.87 \\
\hline & & & $(45)$ & $(0.00-0.08)$ & & $( \pm 0.06)$ \\
\hline
\end{tabular}



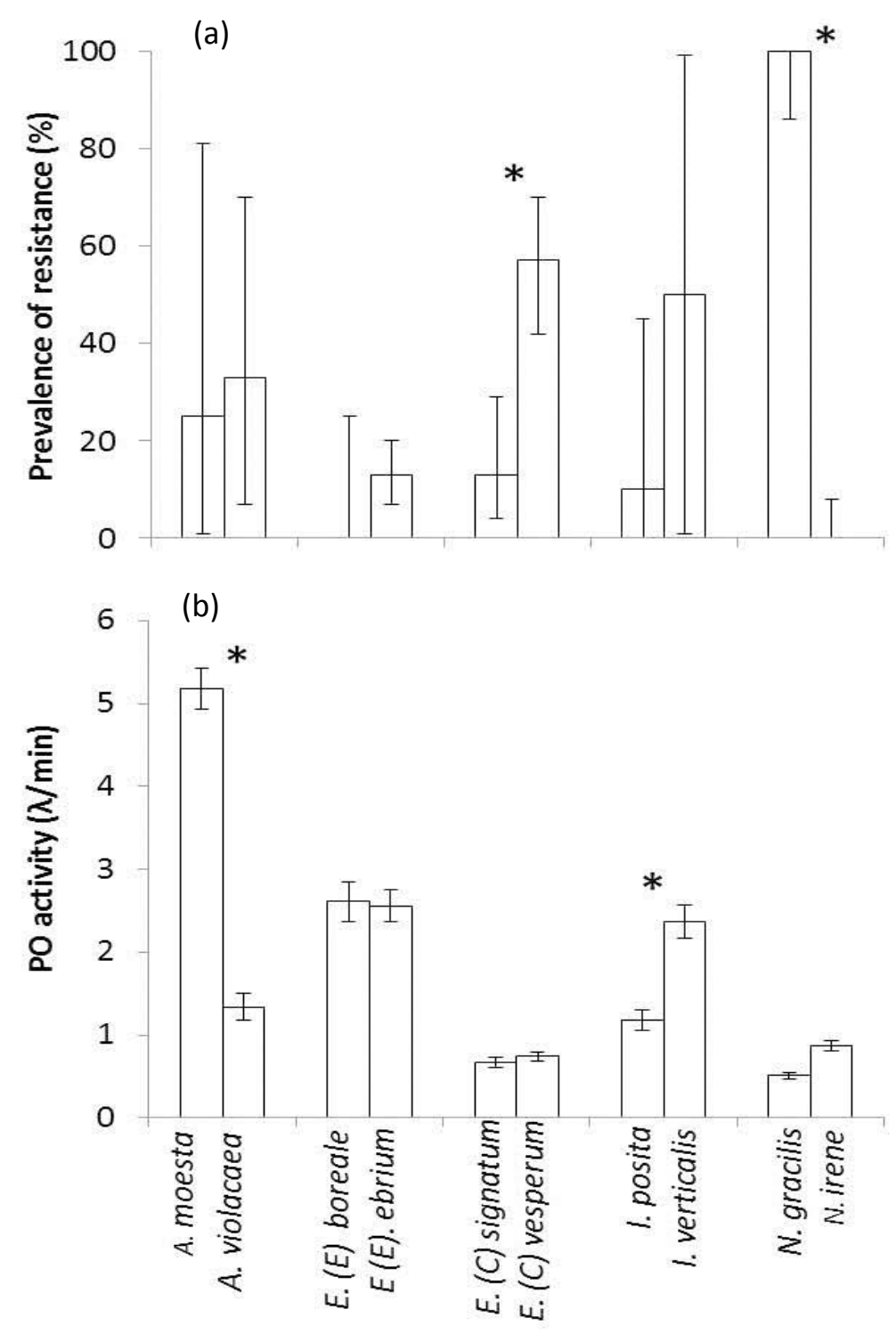

Figure 5.1. Differences between mite resistance (a) and PO activity (b) in ten coenagrionid damselfly species grouped by species pair. Prevalence of resisted mites (error bars $\pm 95 \%$ Clopper-Pearson confidence intervals) is defined as the proportion of infected damselflies which resisted at least one water mite parasite. PO activity (error bars $\pm \mathrm{SE}$ ) is based on mean values from the $\mathrm{PO}$ assay controlled for by thoracic protein content. $*$ = significant differences within a species pair. 
Based on the Fisher Exact two-tailed tests, there was highly significant effect of species on resistance in two species pairs (Table 5.2). In Enallagma (Chromatallagma), E. vesperum is more likely to resist water mites than its close relative E. signatum $(\mathrm{p}<0.007)$ and in Nehalennia, $N$. gracilis is more likely to resist mite parasites than $N$. irene $(\mathrm{p}<0.001)$. In the other three species pairs there was no significant difference in prevalence of resistance (Table 5.2).

Innate immunity (Phenoloxidase (PO) activity)

A total of 427 damselfly individuals were used for the PO and thoracic protein assays (Table 1). PO activity ranged on average from $0.51 \lambda / \mathrm{min}( \pm 0.04 \mathrm{SE})$ in $N$. gracilis to $5.18 \lambda / \min ( \pm 0.25 \mathrm{SE})$ in $A$. moesta (Table 5.1). Based on the ANCOVA analyses, in two of the five species pairs there was significant effect of species on PO activity after controlling for thoracic protein content (Fig. 5.1b). Sex had minimal effect on PO activity in any of the species pairs. Argia moesta had significantly higher PO activity $\left(\mathrm{F}_{1,78}=78.24, \mathrm{p}<0.001\right)$ than $A$. moesta. Similarly, in Ischnura, PO activity was significantly higher $\left(\mathrm{F}_{1,82}=14.66, \mathrm{p}<0.001\right)$ in I. verticalis than in I. posita. There was no effect of species on PO activity in the Enallagma (C.), Enallagma (E.) or Nehalennia species pairs (Table 5.2). Two of these three species pairs are where the significant differences in prevalence of resistance were observed. 
Table 5.2. Fisher exact two-tail test results of the differences in resistance between the species within the species pairs and ANCOVA results of species determining the differences in PO activity within each damselfly species pair. ANCOVA results include the effect of the covariate, thoracic protein content and the interaction between thoracic protein content and species. Significant differences for the species differences are in bold.

\begin{tabular}{|c|c|c|c|c|c|}
\hline \multirow[t]{2}{*}{ Host Sp. pair } & \multirow{2}{*}{$\frac{\text { Resistance }}{\mathrm{P}}$} & \multicolumn{4}{|c|}{ Innate Immunity } \\
\hline & & Effect & df & $\mathrm{F}$ & $\mathrm{P}$ \\
\hline \multirow[t]{3}{*}{ Argia } & 1.00 & Species & 1,78 & 78.24 & $<0.0001$ \\
\hline & & Protein content & 1,78 & 0.02 & 0.89 \\
\hline & & Species $*$ Protein content & 1,78 & 1.32 & 0.25 \\
\hline \multirow[t]{3}{*}{ Enallagma $(\mathrm{C})$} & 0.007 & Species & 1,71 & 0.57 & 0.46 \\
\hline & & Protein content & 1,71 & 0.27 & 0.61 \\
\hline & & Species $*$ Protein content & 1,71 & 0.19 & 0.66 \\
\hline \multirow[t]{3}{*}{ Enallagma (E) } & 0.12 & Species & 1,64 & 0.25 & 0.62 \\
\hline & & Protein content & 1,64 & 0.42 & 0.52 \\
\hline & & Species $*$ Protein content & 1,64 & 3.75 & 0.06 \\
\hline \multirow[t]{3}{*}{ Ischnura } & 0.40 & Species & 1,82 & 14.66 & 0.0003 \\
\hline & & Protein content & 1,82 & 0.01 & 0.91 \\
\hline & & Species $*$ Protein content & 1,82 & 0.0002 & 0.99 \\
\hline \multirow[t]{3}{*}{ Nehalennia } & $<0.001$ & Species & 1,103 & 1.16 & 0.29 \\
\hline & & Protein content & 1,103 & 5.49 & 0.02 \\
\hline & & Species $*$ Protein content & 1,103 & 0.02 & 0.88 \\
\hline
\end{tabular}


Innate immunity versus mite resistance

There was no relationship between PO activity and prevalence of resisted mites across damselfly species $(\mathrm{r}=-0.16, \mathrm{df}=9, \mathrm{P}=0.66)$.

Looking at each species pair separately, there was no consistent pattern (Fig.5.1). In Argia and Ischnura the species with significantly higher PO activity did not resist a greater number of parasites. In Enallagma (Chromatallagma) and Nehalennia, there was no difference in PO activity, but $E$. (C.) vesperum and $N$. gracilis had significantly higher prevalence of resisted mites, as mentioned. Finally, In Enallagma (Enallagma), there was no significant difference in resistance. I could not infer species effects in the ANCOVA for this species pair because of the near significant interaction between thoracic protein content and species. Interestingly, the slope of the interaction between thoracic protein content and PO activity was positive in E. boreale whereas the slope was negative in $E$. ebrium.

Innate immunity and resistance versus geographic range

All the species pairs, except one (Enallagma $(E)$ ) showed a significant difference in either resistance or PO activity. Resistance was significant in two species pairs (Enallagma $C$ and Nehalennia) where the species with the smaller geographic range size (E. vesperum and N. gracilis respectively) demonstrated higher resistance levels (Fig 5.3; Table 5.1). In Ischnura the species of the pair with a larger geographic range (I. verticalis) showed significantly higher levels of PO activity (Fig. 5.4). The opposite was 
observed in the Argia species pair where A. violaceae, the less widespread species, had significantly lower PO activity than $A$. moesta (Fig. 5.4)

Thus in all of the cases, sibling host species with differences in size of geographic range differed in either resistance or PO activity.

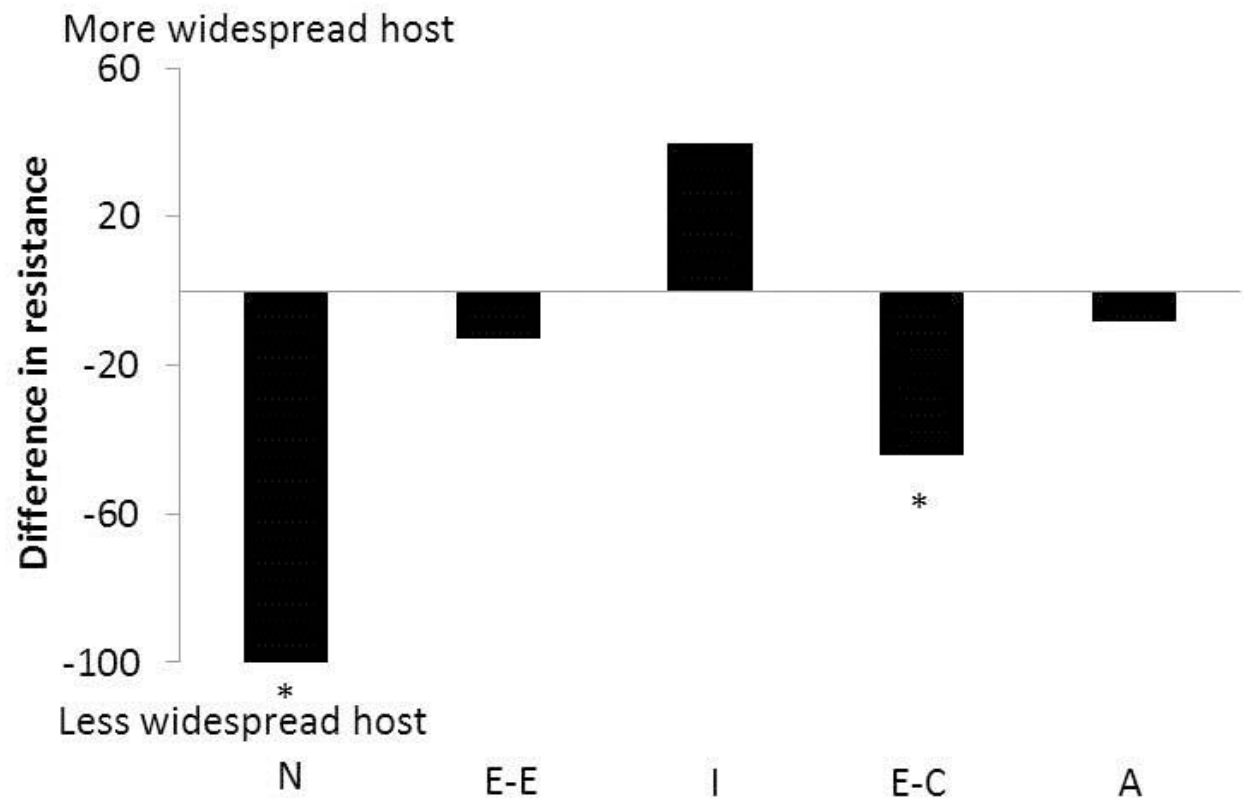

Figure 5.2. Within species pair difference in measures of water mite resistance between species. A positive difference means that the more widespread species of the species pair has higher estimate of prevalence of infection (from Table 5.1); a negative difference means that the less widespread species has higher estimate of prevalence. Actual significant differences in prevalence values are marked by *. The organization of the species pairs reflects geographical range differences within the species pair from most different to least different. Key: A, Argia; E-C, Enallagma Chromatallagma; E-E, Enallagma Enallagma; I, Ischnura; N, Nehalennia. 


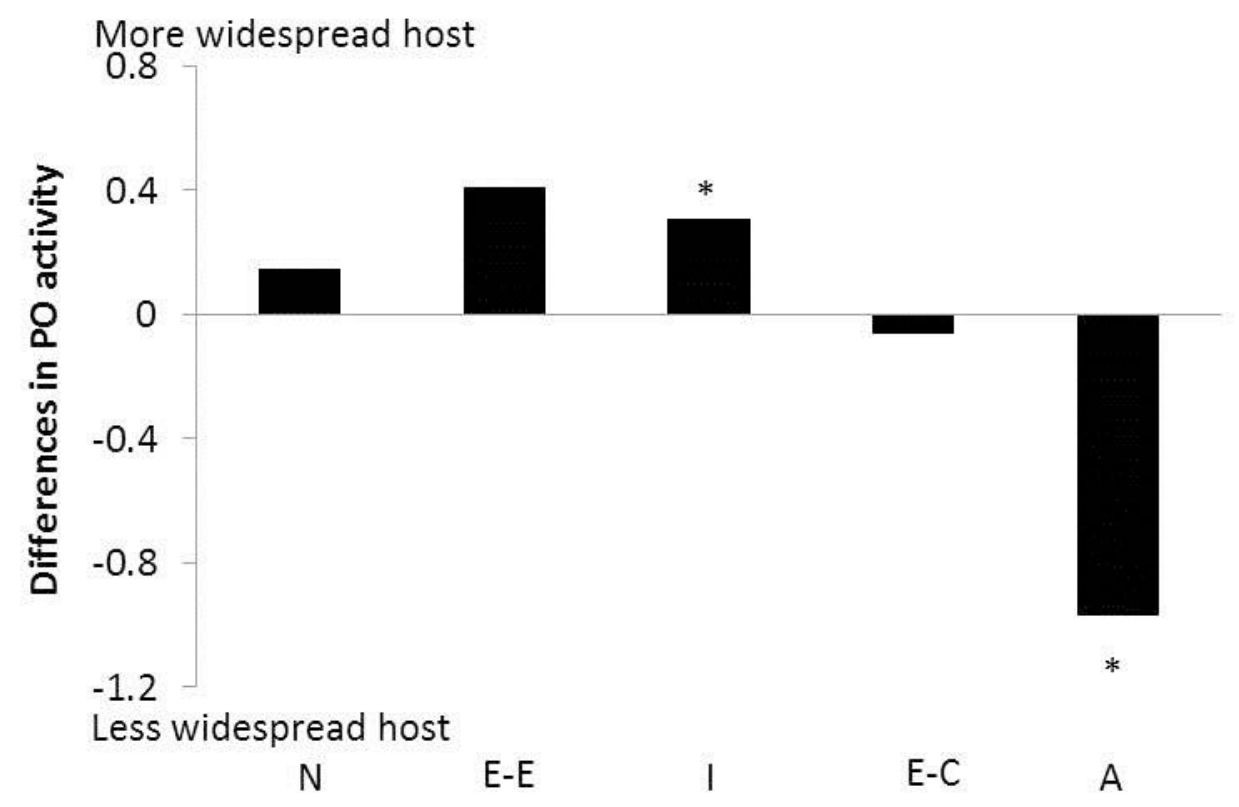

Figure 5.3 Within species pair difference in measures of PO activity between species. A positive difference means that the more widespread species of the species pair has higher estimate of prevalence of infection (from Table 5.1); a negative difference means that the less widespread species has higher estimate of prevalence. Actual significant differences in prevalence values are marked by * The organization of the species pairs reflects geographical range differences within the species pair from most different to least different. Key: A, Argia; E-C, Enallagma Chromatallagma; E-E, Enallagma Enallagma; I, Ischnura; N, Nehalennia. 


\section{DISCUSSION}

The main question of this study was the following: does interspecific variation in a constitutive measure of innate immunity predict interspecific variation in resistance for closely related species? The results show no clear relationship between PO activity and resistance across a range of related host species parasitized by same or similar parasites species. Within host species pairs, a species with higher prevalence of resisted mites is not the same species as the one with higher PO activity. Within the genera Argia and Ischnura, one species had significantly higher PO activity but resistance levels were not different between the two species in each species pair. Interestingly, even though closely related species have more similar levels of PO activity, this measure of innate immunity seems evolutionary labile between genera. Both Nehalennia and Enallagma (Chromatallagma) species had an order of magnitude less PO activity than the other three species pairs. However, this is not consistent given the overlap in PO activity between Nehalennia and Enallagma (Chromatallagma). Additionally, there is variation in PO activity as in the two Argia species and overlap in PO activity in the species pairs, i.e. Argia, Enallagma (Enallagma) and Ischnura.

In this study I did not find a relationship between PO activity and prevalence of resisted mites within these populations but there are potential life history factors that could explain the variability that I see in both variables within species pairs. Given that variability in species characteristics has been demonstrated to be an important driver of immune function (Armitage and Siva-Jothy 2005, Rynkiewicz et al. 2013), I expected that such variability might explain some of my observed patterns in a similar way. My 
focal species were chosen because internal and external parasitism levels have been documented before and there are ecological differences, such as geographic range, in host species characteristics between phylogenetically closely related species (see Mlynarek et al. 2012, Mlynarek et al. 2013). The pattern between geographic range with either resistance and PO activity was not clear. In the cases where there was significant difference in resistance (two of five species pairs), the species with the smaller geographic range size resisted water mites more readily. However, the more widespread species tended to have higher levels of PO activity of $75 \%$ of the cases where there was significant difference in PO activity.

The main interspecific differences documented within the species pairs that could potentially explain differences in either prevalence of resistance or PO activity are cuticle coloration, parasite pressure and geographic range. In Argia and Enallagma (C.) cuticular coloration may explain the differences in either PO activity or resistance. Argia moesta is larger, darker and has higher PO activity than A. violacaea. This is an expected result considering that darker mealworm beetles had higher immune capacity than their paler counterparts (Armitage and Siva-Jothy 2005). In the case of Enallagma $(C$.) there may be a trade-off between cuticular coloration and prevalence of resistance. Enallagma vesperum is parasitized and resists water mites much more readily than E. signatum (Mlynarek et al 2013) but E. signatum is orange rather than yellow. In Ischnura differences in PO activity may be due to the parasite pressure on the more ubiquitous species I. verticalis. It has been suggested that with increasing the representation across its range, the number of interspecific interactions will increase and the species will 
require more general protection from a greater assortment of parasites (Morand et al. 2010). Finally, N. gracilis resists the parasites (c.f. Mlynarek et al. 2014) whereas $N$. irene does not. Nehalennia gracilis may be evolved to recognize parasites that are similarly specialised to the same particular habitat.

Variation in immune response and resistance has been documented in many studies examining the costs and benefits of resistance to pathogens such as bacteria (Wilson and Cotter 2013). In two species of wild rodents, Rynkiewicz et al. (2013) demonstrated that host age, sex and species as well as specificity of the parasites are drivers of increased bacterial killing assay, a proxy to test individual innate immunity. In invertebrates, studies demonstrate that genetic heterogeneity influences resistance to pathogens between distinct populations of Drosophila (Corby-Harris and Promislow 2008), Daphnia (Pauwels et al. 2011), Bombus (Whitehorn et al. 2011) and Calopteryx (Kaunisto et al. 2013). My study differs from those studies because I include variation in innate immunity and resistance between different host species and not different populations within a species. Additionally, resistance in these studies is not as easily observable as for the damselfly-water mite host-parasite system because resisted water mites remain on the damselfly. Recently, a study compared response to nylon inserts, an induced immune elicitor, in two differentially resisting lestid damselflies (Nagel et al. 2011). The authors found that Lestes forcipatus Rambur, the species that resists its parasites more readily, melanises the inserts more than the one with lower resistance, Lestes disjunctus Selys. In the case of lestid damselflies, it seems that a species resistance and its response to induced immune elicitors are linked when the specificity of the 
challenge is controlled for. Likewise in my study, parasite might have evolved evasion of recognition by some host species' immune system and not by others because of the coevolutionary history between certain damselfly host-Arrenurus parasite associations.

There are caveats for using PO activity as an appropriate measure of innate immunity in insects especially in damselflies. Gonzalez-Santoyo et al. (2012) reviewed the success of PO activity in insect immunity; based on the literature, 11 of 23 papers that studied PO activity in terms of innate immunity found a positive relationship between PO activity and successful pathogen defence. Of the remaining 12 papers, half found a negative relationship and the other six found no relationship. The main explanation for this variability is suggested to be the high costs of this defence mechanism and its dependence on resources acquired in the past by the host. Recently, Moreno et al. (2013) tested the range of currently used proxies of immune response including PO activity to provide methodological standards depending on the study undertaken. Their major issue with PO activity was that with long term storage, even in $-70^{\circ} \mathrm{C}$ temperature, $\mathrm{PO}$ activity could be degraded or spontaneously activated. There is no question that PO activity plays a role in insect innate immunity but it is very species specific. There is a debate about the relevance of PO activity as a proxy for innate immunity because the PO cascade is also used for different functions such as cuticular melanisation, wound healing, and female egg tanning (Sugumaran et al. 2000, Bidla et al. 2009). Variation in storage was controlled for in this study (i.e. all lab analyses were performed within six months after capture). However, subtle differences between related host species in traits like degree of cuticular melanisation and its effects of PO activity remain unknown. 
There are issues of studying response in multiple phylogenetically related host species because of the problem of controlling for variation in ecology between species that might influence any trait studied. However, these types of studies are important because they may lead to an added understanding of species interactions. I was limited to a single population of each host species because the host species within the species pairs had to be sympatric. Such populations share ectoparasitic mites (Mlynarek et al. 2013) which may not be the case if host species were collected from different sites. At a particular site, there is added probability that two closely related species are infected by the same parasite species so the responses to parasitism and the levels of innate immunity will under the same pressures for both closely related host species.

In conclusion, I found no correlation between constitutive measures of innate immunity and resistance to parasites among damselflies species within a population. The damselfly-water mite associations is an appealing study system to test questions of resistance which is easy to document. In comparison to other insects, PO activity may not be a good proxy for innate immunity in damselflies. The validity of PO in insect immunity is not questioned, but one does question its precise role in elicitation of a melanisation defence. Ham et al. (1995) speculated that the main roles of PO in blackflies may be cuticle formation. Even though melanisation as an immune function is documented in damselflies, investment in PO is likely to be traded off with other uses. As Gonzalez et al. (2012) suggest, the knowledge of ecological immunology is ever increasing, but we do not yet have the full understanding on PO activity as the sole or even best substrate to use. It may be that innate immunity relates to levels of resistance 
after controlling for other factors. I encourage future studies of other invertebrate taxa to make investigations into other factors such as host recognition, rather than relying on one or more measures of innate immunity in isolation, as is often the case. Such studies will help identify some of the constraints on immune system evolution and function. 


\section{CHAPTER SIX}

\section{Explaining susceptibility and resistance to a multi-host parasite}

This chapter formed the basis for the following publication and was reproduced with permission from the publisher, (C) 2014 Springer, Inc:

Mlynarek, J.J., Knee, W. and M.R. Forbes, 2014. Explaining resistance in a multihost parasite. Evolutionary Biology 41: 115-122 


\section{ABSTRACT}

Closely related host species are known to show variation in the level of resistance towards the same or similar parasite species, but this phenomenon is understudied. Such studies are important for understanding the ecological factors that might promote susceptibility or resistance to parasites: in particular, whether one host species is a larger target of selection for the parasite by virtue of being more abundant locally or more regionally widespread than another host species. In this study, I examined the expression of resistance by two closely related species of damselflies (Nehalennia irene and Nehalennia gracilis) against an Arrenurus water mite species. I show that the host species at each of two isolated sphagnum bogs have statistically indistinguishable levels of prevalence and intensity of infection by mite larvae. Despite having similar measures of parasitism, the regionally less represented species ( $N$. gracilis) showed total resistance, whereas the regionally well-represented species ( $N$. irene) was completely susceptible. Moreover, the form of resistance expressed by $N$. gracilis was unique, in that the oral glands of the mite were melanised. Also, this mite species was not found outside of isolated bog habitats. These results suggest that there might have been strong historical selection from this mite on the bog specialist, $N$. gracilis, and that this selection may have resulted in resistance evolving to fixation in a series of isolated populations. 


\section{INTRODUCTION}

The dynamic interaction between a single species of parasite and a given host species is often studied in the context of an antagonistic coevolutionary arms race, whereby the participant species counter each other's adaptations of defense or attack (Davies et al. 1989, Foitzik et al. 2001, Langmore et al. 2003). There is a need to document the strength of arms races in host-generalist parasite associations; such associations are common in nature. Generalist parasite species are those species capable of exploiting a wide range of host species (Price 1980, Poulin 2007). Considering that generalist parasite species may have to cope with the defense mechanisms of several to many host species, it is possible that generalists are poorly equipped to compete in an arms race with any given host species (Whitlock 1996). Stated more formally, the selective pressure to adapt to a single host species is likely more relaxed for a generalist than for a specialist parasite species (Lajeunesse and Forbes 2002).

Past studies have documented significant interspecific variation in successful parasitism. For example, generalist hen fleas are often more abundant (or successful) on certain avian host species, depending on host ecology (Tripet and Richner 1997). In another study, a generalist water mite species was more successful on the dragonfly Sympetrum obtrusum than on the closely related host species Sympetrum internum (Forbes et al. 1999). More recent studies have provided potential explanations for interspecific variation in parasite success. Such variation has been studied in relation to interspecific variation in immune expression (Yourth et al. 2001), home range size 
(Bordes et al. 2009), geographic range (e.g., Tella et al. 1999) or local abundance (Teder and Tammaru 2003) of host species.

There is also evidence showing that more widespread host species experience higher measures of parasitism (prevalence and intensity) at a particular site (Tella et al. 1999). However, Mlynarek et al. (2012) found that less widespread sibling host species often had higher prevalence and/or intensity of parasitism by generalist gregarine parasites than did more widespread host species; this pattern was seen across half of the damselfly hostgregarine species associations investigated. In the other half of associations, there were no differences between more and less widespread host species in their levels of parasitism. One of the key issues emerging from that study was whether the range or local abundance of the different host species influenced measures of parasitism or resistance to parasites.

In other studies, it was suggested that a host species with a lower local population density (Lopez 2005) and smaller regional representation (Thompson 2005) would have fewer parasite individuals ( $c f$. Grant and Samways 2007). Less widespread and less wellrepresented host species might be behaviorally more adapted to evade particular parasites or might have higher immune function to ward off those parasites. Alternatively, one could speculate that the parasite species could track subtle differences in host phenology for host species differing in relative abundance, making the parasite more likely still to encounter the more common host species, to which it adapts in other ways (Yourth et al. 2001). As a result of their lower exposure to parasite individuals, less widespread or less well-represented host species may not impose such selection pressure on parasites. Those 
parasites might be more easily recognized as non-self by such hosts; therefore, I might expect greater specific host resistance by less represented or less widespread hosts.

Damselflies and their parasitic water mites provide a model system to explore the extent to which measures of parasitism and resistance vary between closely related host species (Grant and Samways 2007, Nagel et al. 2010). Water mite species are narrow to broad host generalists, feeding on a few versus multiple host species within a given suborder of insects (Smith et al. 2010). Larval water mites are external parasites, piercing the cuticle of their host as the host ecloses. They engorge during the pre-reproductive phase of the host, and it is easy to distinguish between resisted and non-resisted water mites by the presence/absence or level of engorgement (Smith et al. 2010). Additionally, prevalence and intensity of parasitic water mites varies across damselfly species, for example, from $3.5 \%$ in Cieragrion glabrum to $38 \%$ in Ischnura senegalensis (Grant and Samways 2007).

My objectives were to explore the extent to which measures of parasitism (prevalence and intensity) of larval water mites differ between two closely related species of damselflies. More specifically, I first examined whether the two species differed in their local abundance and in the prevalence or intensity of infestation by one or more unnamed mite species. I then determined whether the water mites infecting both host species were of the same species because my ideas about specific resistance required it to be the same species. Finally, I examined whether one host species showed greater specific resistance to mites than the other host species, as predicted based on the logic above. 


\section{METHODS}

\section{Host distribution and abundance}

I focused on two damselfly species, Nehalennia irene and Nehalennia gracilis, which are phylogenetically closely related (Carle et al. 2008), of similar size, and have similar ecologies (Walker 1953). However, the geographic range (Abbott 2007) and regional representation (Van Gossum et al. 2007) is greater for $N$. irene than for N. gracilis. Local abundance at sites also varies between these two species; usually $N$. irene is found in greater numbers when both species are present at a site (Van Gossum et al. 2007; but see below).

Nehalennia irene and $N$. gracilis are restricted to the Nearctic region. The difference in geographic range between $N$. irene and $N$. gracilis was determined using the Odonata Central database (Abbott 2007). As in Mlynarek et al. (2012), the geographic range of a species was determined by noting its presence or absence on a $2^{\circ}$ latitudelongitude grid of cells overlaid on geographic data. The areas of all the cells where the species was 'present' were summed to produce a measure of the entire geographic range of a particular species (see Hassall 2012 for details).

The difference in regional representation of each species in southeastern Ontario and eastern Quebec was determined using personal observations and Van Gossum et al. (2007). Van Gossum et al. (2007) collected at 21 sites within the Saint Lawrence Lowlands and noted the presence of each host species at each site. An additional 13 sites were surveyed through personal observation for presence and absence of $N$. irene and $N$. 
gracilis. The proportion of sites occupied by a particular species can therefore become a proxy of regional representation. Regionally, $N$. irene occurs at many more sites than does $N$. gracilis, which is a habitat specialist of sphagnum bogs (Walker 1953). Van Gossum et al. (2007) collected N. gracilis at only 4 of the 21 sites that they examined, whereas $N$. irene was collected at all of the sites. Similarly, in a related study designed to include bog habitats, $N$. irene was collected at 13 sites but $N$. gracilis was collected at only 6 sites (Chapter 4).

Relative local abundance of damselflies was determined at the two isolated study sites using a standardized sampling protocol. Nehalennia irene and N. gracilis were collected once a week from 6 June -18 July 2011 at the two sites in eastern Ontario. The two sites were located $20 \mathrm{~km}$ apart: Westport Bog $\left(44^{\circ} 42^{\prime} 38^{\prime \prime} \mathrm{N} 76^{\circ} 22^{\prime} 30^{\prime} \mathrm{W}\right)$ and Hebert Bog $\left(44^{\circ} 29^{\prime} 50^{\prime \prime} \mathrm{N} 76^{\circ} 24^{\prime} 56^{\prime} \mathrm{W}\right)$. At each weekly visit, I determined relative host abundance by sweep net collecting of individuals of both species for 20 minutes, along the margin of the bog pond. Collecting for 20 minutes has been shown to provide a good representation of the local assemblage of damselflies (Bried et al. 2012). Two ANOVAs were performed to test whether the relative local abundance was different between $N$. irene and $N$. gracilis at each of the two sites. After collecting for 20 minutes, further collecting continued until at least 30 individuals of each focal host species were caught. All damselfly specimens collected were stored in $95 \%$ ethanol for later species identification, and for enumeration of live and dead (unengorged) mites. 
Prevalence and intensity of parasitism

All host individuals were inspected for larval water mites in the lab using a dissecting microscope (Zeiss SteREO Discovery .V8). Hosts were scanned for mites on the venter of the thorax and abdomen at $20 \mathrm{X}$ power. This allowed both live engorged or engorging mites, and dead (resisted) mites to be enumerated. I then tallied mite prevalence and intensity by site, species, host sex and collection date. Mites other than those of the genus Arrenurus (e.g., Limnochares) were not enumerated for this study because they infected only $N$. irene hosts in the last two weeks of sampling with a prevalence of less than $1 \%$.

Prevalence is defined as the proportion of sampled hosts infected by at least one individual parasite, and intensity is the average number of parasite individuals on infected hosts (Rózsa et al. 2000). I used two separate Fisher's exact tests to compare the parasite prevalence of $N$. irene and $N$. gracilis at each site. I used two Bootstrap 2-sample t-tests (using QP3.0: Rózsa et al. 2000) to compare mean intensity of parasitism between the host species at each site.

\section{Mite identification}

\section{$\underline{\text { DNA barcoding }}$}

Ten water mites were randomly selected for DNA extraction: eight extractions (two mites from each host species at each site) were successful. Total genomic DNA was extracted from whole specimens for 24 hours using a QIAmp DNA Mini Kit (Qiagen Inc., Santa Clara, CA, USA) following the protocol of Knee et al. (2012). PCR 
amplifications were performed in a total volume of $25 \mu 1$, with $14.7 \mu 1 \mathrm{ddH}_{2} \mathrm{O}, 2.5 \mu 110 \times$ ExTaq buffer, $0.65 \mu 125 \mathrm{mM} \mathrm{MgCl}_{2}, 1.0 \mu 1$ of each $10 \mu \mathrm{M}$ primer, $2.0 \mu 110 \mathrm{mM}$ dNTPs, $0.15 \mu 1$ ExTaq DNA polymerase (Promega Corp., Madison, WI, USA), and $3 \mu 1$ genomic DNA template. PCR amplification cycles were performed on an Eppendorf ep Gradient S Mastercycler (Eppendorf AG, Hamburg, Germany). Primer pairs AL F2 (5'AGCAAGTTTAAGAAGTCT-3') + AL R2 (5'-ATTTTTATCACCACTATCC-3') were used to attempt to amplify a $491 \mathrm{bp}$ fragment of the 5'-end of COI of Arrenurus larvae and not their damselfly hosts. The thermocycler protocol for COI amplification was as follows: initial denaturation cycle at $94^{\circ} \mathrm{C}$ for $3 \mathrm{~min}$, followed by 45 cycles of $94^{\circ} \mathrm{C}$ for 45 s, primer annealing at $40^{\circ} \mathrm{C}$ for $45 \mathrm{~s}, 72^{\circ} \mathrm{C}$ for $1 \mathrm{~min}$, and a final extension at $72^{\circ} \mathrm{C}$ for 5 $\min$.

Amplified products and negative controls were visualized on $1 \%$ agarose electrophoresis gels, and purified using pre-cast E-Gel CloneWell 0.8\% SYBR Safe agarose gels (Invitrogen, Carlsbad, CA, USA) following Gibson et al. (2010). Sequencing reactions followed the protocol of Knee et al. (2012), and sequencing was performed at the Agriculture \& Agri-Food Canada, Eastern Cereal and Oilseed Research Centre Core Sequencing Facility (Ottawa, ON, Canada).

Sequence chromatograms were edited and contiguous sequences were assembled using Sequencher v4.7 (Gene Codes Corp., Ann Arbor, MI, USA). COI sequences were aligned manually in Mesquite v2.74 (Maddison and Maddison, 2010) according to the 
translated amino acid sequence. Sequences have been submitted to GenBank (KC562147 - KC562154).

\section{$\underline{\text { Morphometrics }}$}

I prepared 17 water mites from $12 \mathrm{~N}$. irene individuals (six from Westport bog and six from Hebert bog) and ten water mites from seven $N$. gracilis individuals (four from Westport bog and three from Hebert bog) for morphometric analysis. The water mites that were used for DNA extraction also were measured. Following DNA extraction, four mites from $N$. irene and three mites from $N$. gracilis were recovered from the extraction buffer. A total of 25 of the 27 water mites were slide-mounted successfully in a polyvinyl alcohol medium, and slides were cured on a slide warmer at about $40^{\circ} \mathrm{C}$ for 2 days. Each water mite was photographed three times using a ZEISS Imager M2 compound microscope with a AxioCam HRm camera and imported using AxioVs40 v.4.8 program. Three photographs of each water mite were taken: a habitus photo (10X zoom), and two photos of taxonomically informative characters including the dorsal shield (20x zoom) and excretory pore (40x zoom) (Zawal 2008, Smith et al. 2010).

The 96 images were imported into ImageJ (Schneider et al. 2012) and rotated so that all the images were orientated in the same direction. A tps file was created using tpsUtil and landmarks were created in the program tpsDig (Rohlf 2009). For the shield photo, 14 landmarks were chosen. For the excretory pore, five landmarks were chosen. The file was then imported into MorphoJ (Klingenberg 2011). To compare whether the 
two host species had different mites based on morphometric measurements, I conducted a discriminate function analysis (DFA) where host species was the classifier.

Nature of resistance

Resistance was assessed while examining damselfly hosts for larval water mites. Resisted mites are dead and remain attached to the host. Dead mites are darker brown in color than live mites and are unengorged, i.e., the edges of their sclerites are connected and not separated by membranous tissue. Live mites, in contrast, are red with ventral sclerites separated by membranous tissue. Once the water mite was assessed as either resisted or not resisted, the nature of resistance (melanisation) was examined using the slide-mounted specimens that were used also for morphometric analysis.

\section{RESULTS}

\section{Host distribution and abundance}

A total of 949 Nehalennia damselflies were collected and examined for parasitism and resistance, at both study sites. At Hebert bog, $N$. irene was more abundant $(10.33 \pm$ $1.63 \mathrm{SD})$ than $N$. gracilis $(4.17 \pm 1.08)$ for the seven week period, when I assessed number of individuals collected per 20 minute sweeps $\left(\mathrm{F}_{1,12}=9.99\right.$, $\mathrm{p}=0.01$; Fig. 6.1). At Westport bog, the difference in relative local abundance between $N$. irene $(6.67 \pm 1.15)$ and $N$. gracilis $(7 \pm 1.63)$ was negligible for the same time period $\left(\mathrm{F}_{1,12}=0.03, \mathrm{p}=0.86\right.$; Fig. 6.1). 


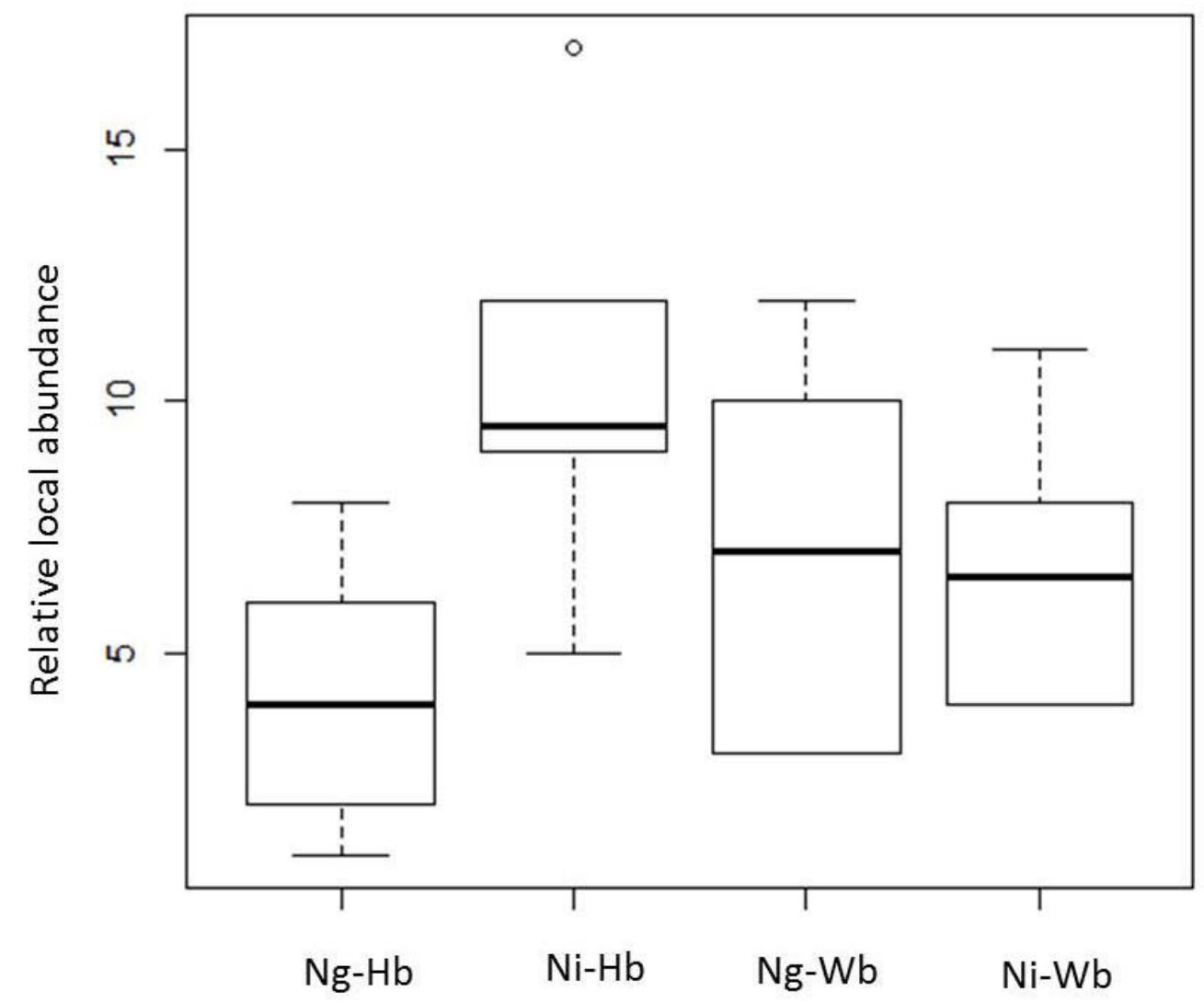

Figure 6.1. Relative local abundance differences between $N$. gracilis $(\mathrm{Ng})$ and $N$. irene (Ni) at Hebert bog $(\mathrm{Hb})$ and Westport bog $(\mathrm{Wb})$ across seven weeks of collecting. Circle represents an outlier, whiskers represent the maximum and minimum values, boxes delimit the upper and lower $25 \%$ quartiles, the dark line represents the median of the local abundance values. 
Table 6.1. Prevalence (\%) with $95 \%$ confidence interval (CI) and median intensity with confidence limit and actual confidence level (CL) of mites parasitizing two focal and two transient host species in Hebert and Westport bogs

\begin{tabular}{l|lccc}
\hline Site & Species & N & $\begin{array}{c}\text { Prevalence \% } \\
(\mathbf{9 5 \%} \mathbf{C I})\end{array}$ & $\begin{array}{c}\text { Mean intensity } \\
\mathbf{( 9 5 \%} \text { CI) }\end{array}$ \\
\hline Hebert bog & N. irene & 284 & $\begin{array}{c}15.8 \\
(11.8-20.6)\end{array}$ & $\begin{array}{c}(1.7-3.2) \\
\end{array}$ \\
& N. gracilis & 208 & $\begin{array}{c}12.0 \\
(6.9-18.9)\end{array}$ & 2.1 \\
& & & 10.3 & 2.6 \\
\hline Westport bog & N. irene & 224 & $(6.6-15.0)$ & $(1.7-4.0)$ \\
& & & 15.5 & 2.1 \\
& \multirow{2}{*}{ N. gracilis } & 233 & $(11.4-20.8)$ & $(1.7-2.7)$ \\
\hline
\end{tabular}


Prevalence and intensity of parasitism

In total, $15.8 \%$ of the $N$. irene (44/284), and $12 \%$ of the $N$. gracilis $(25 / 208)$ collected in Hebert bog had at least one mite (Table 6.1). At Westport bog, 10.3\% of the $N$. irene $(23 / 224)$ and $15.5 \%$ of the $N$. gracilis collected $(36 / 233)$ were infected by at least one mite (Table 6.1). The intensity of infection varied between $1-11$ water mites on $N$. irene, and $1-7$ on $N$. gracilis in Westport bog. In Hebert bog, infection intensity ranged from $1-18$ on $N$. irene and $1-11$ on $N$. gracilis (Table 6.1).

Based on the Fisher's exact tests, prevalence of infestation did not differ between the two host species at either site (Hebert bog: $p=0.24$; Westport bog: $p=0.12$; Table 6.1). I also found that median intensity did not differ between the two host species at each site (Hebert bog: $\mathrm{t}=-0.17, \mathrm{P}=0.87$; Westport bog: $\mathrm{t}=-0.75, \mathrm{P}=0.47$; Table 6.1).

\section{Mite Identification}

\section{DNA Barcoding}

A 462 bp fragment of COI was successfully amplified from eight individuals (two from each species at each site). The level of divergence between these mites was low, with an average pairwise distance of $0.3 \% \pm 0.2 \%$, and a range of $0-0.6 \%$. Additionally, there was no tendency for mites from a particular host species or site to cluster together. Public searches on GenBank and Barcode of Life Data Systems taxonomy browser provided a closest match of $86.93 \%$ similarity between these specimens and Arrenurus reflexus. 


\section{$\underline{\text { Morphometrics }}$}

The quantitative morphometric data also suggests that these mites represent a single species. The output of DFA was not significant for the comparisons of water mites from the two host species in either the 14 landmarks in the shield $\left(t^{2}=435.26, p=0.174\right)$ or the five landmarks in the excretory pore $\left(\mathrm{t}^{2}=15.55, \mathrm{p}=0.09\right)$. Two other species of damselflies, Enallagma ebrium and Ischnura verticalis, were also parasitized by the same Arrenurus species based on morphological identification (shape of the dorsal shield, engorgement size in comparison to the $N$. irene mites and shape and size of excretory pore). I suggest that the parasite is a generalist as it does not discriminate between host species.

Nature of resistance

In total, 61 of the $441 N$. gracilis hosts examined had mites. All of these mites were un-engorged and dead showing evidence of being resisted. In contrast, 67 of the 508 $N$. irene hosts examined had mites, and none of those infested hosts resisted any of the mites (Fig. 6.2a). All slide-mounted water mites from N. gracilis hosts showed the same pattern of resistance, in which areas around the mouthparts as well as areas within the mite, most likely the oral glands (Mitchell 1955), were melanised (Fig. 6.2b). This suggests that once the water mite is recognized, the host melanises the oral glands of the mite. 


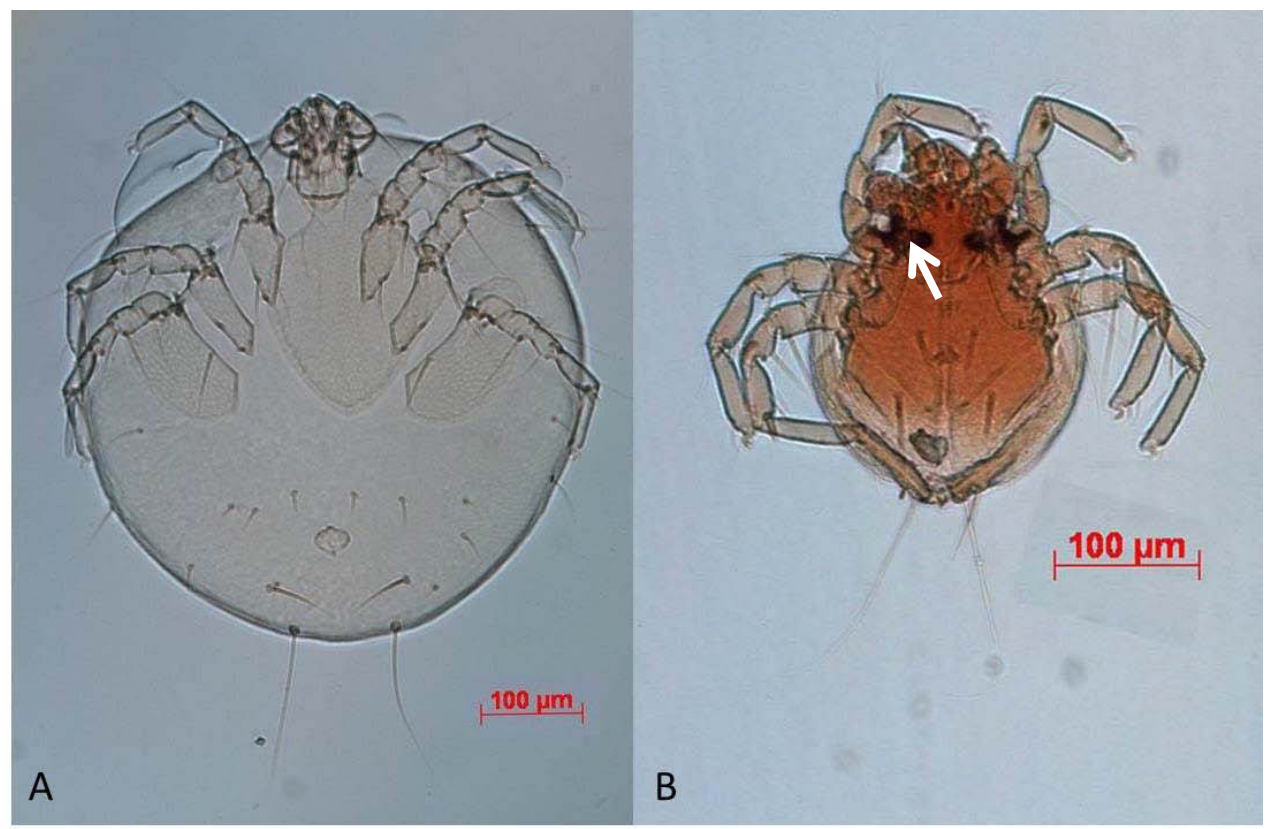

Figure 6.2. (A) Engorged Arrenurus sp. from N. irene, and (B) resisted Arrenurus sp. from N. gracilis. Arrow indicates the melanization of the oral gland region.

\section{DISCUSSION}

The two host species that I studied differ in their range, regional representation, and habitat specialization. Nehalennia irene has a widespread distribution $\left(4.03 \times 10^{6}\right.$ $\left.\mathrm{Km}^{2}\right)$ whereas $N$. gracilis has a narrower distribution $\left(1.51 \times 10^{6} \mathrm{Km}^{2}\right)($ Mlynarek et al. 2012). Nehalennia gracilis is a bog specialist, and is restricted in its regional abundance (Walker 1953). In contrast $N$. irene is a habitat generalist; it is a cosmopolitan species present at most lentic aquatic habitats (Walker 1953). Regionally, N. irene occurs in more locations and habitat types than N. gracilis (Van Gossum et al. 2007). At four sites where the host species were sympatric, $N$. irene was constantly more locally abundant (Van Gossum et al. 2007). Although my collecting protocol differed, I found similar results at 
Hebert bog to that of Van Gossum et al. (2007), but not at Westport bog, where $N$. gracilis and $N$. irene were similarly abundant (Fig. 1). Iserbyt et al. (2010) showed that $N$. irene has lower population genetic structure than does $N$. gracilis, probably because $N$. gracilis populations are more isolated from one another with lower effective population sizes (i.e., the effect of genetic drift should be stronger).

There are otherwise many similarities between the two sibling host species. The two species are of similar size. Both host species have a similar emergence times and have similar lengths of flight seasons where the first individuals of both species can usually be recorded in the last week of May; the flight seasons of both species continue into August (Walker 1953).

Based on past studies, even closely related damselfly species with similar ecologies demonstrate variable infection rates (Forbes et al. 1999, Grant and Samways 2002, Nagel et al. 2011), but this could be due to different species of mites infecting different host species. However in this study, the lack of morphological differences and low genetic divergence between mites collected from different host species provides good evidence that mites from these two host species represent a single species of Arrenurus. In many groups of mites the barcoding region of COI is fast evolving, with $18 \%$ COI divergence between stream and lake-inhabiting populations of the water mite Hygrobates nigromaculatus (Martin et al. 2010), and an average of 16\% divergence between closely related species of Nicrophorus-associated Uropodoidea (Knee et al. 2012). Thus, it could be expected that if the mites from $N$. irene and $N$. gracilis were 
distinct species they would show a greater level of divergence than that observed $(0.2 \%)$. In this damselfly-water mite species study system, I have good evidence now to suggest that the water mite attacks both host species indiscriminately because of the lack of host species differences in measures of parasitism.

Interestingly, one of the two host species shows complete resistance to the parasite and the other species is wholly susceptible. A similar pattern of resistance has been documented only once previously, in which Arrenurus planus successfully engorges on Sympetrum obtrusum dragonflies but is most often resisted on a closely related host species, Sympetrum internum, which is the less locally abundant species (Forbes et al. 1999). Typically, resistance towards water mites is partial, with a few water mite individuals being resisted on a particular individual host (Yourth et al. 2002, Nagel et al. 2010).

Odonate resistance towards water mites can be easily documented because of the nature of the immune response, which involves melanisation. Once a water mite finds a host, it embeds its chelicerae and forms a stylostome. If the host recognizes the infection by the parasite, it can melanise the stylostome thus starving the water mite (Forbes et al. 1999). The dead water mite will remain attached to the host because the melanised stylostome and attached chelicerae remain embedded in the host (Forbes et al. 1999, 2002). In my study system, the stylostome is melanised but it seems that the melanisation also occurs within the water mite at the mites' oral glands (Fig. 2B). We know that the two species have similar phenoloxidase (PO) activity (Chapter 5). Phenoloxidase is an 
enzyme whose activation results in the production of melanin and its levels are generally used as a measure of general innate immune ability in insects (Gonzalez-Santoyo and Cordoba-Aguilar 2012). I suspect that the observed species differences are due to differences in specific immunity and not general innate immunity.

Forbes et al. (1999) proposed three related factors that should help maintain specific resistance expressed by one or a few host species; host relative abundance, the novelty of the defense, and the success of the parasites on alternative hosts. My results bear strongly on these factors proposed by Forbes et al. (1999). First, the type of the defense shown by $N$. gracilis is novel in that the melanisation is also found within the water mite, and mites appear to not have evolved counter defenses against this novel resistance. Second, the water mite is successful on alternative hosts, so hosts showing novel resistance might not be large targets for selection. Furthermore, my system also demonstrates that the less represented host species (and often the less abundant species, following Van Gossum et al. 2007) is the one that resists the parasite and is a habitat specialist.

It is possible that as a habitat specialist, $N$. gracilis may have evolved recognition for this particular parasite species. Nehalennia gracilis only has to recognize bogrestricted parasites, whereas $N$. irene has to contend with parasites from many different types of habitats (and might actually have higher innate immunity). Simková et al. (2001) presented data that as a fish host's regional representation increases, they must contend with more parasite species. Additionally, more ecologically specialized host species have 
been reported to have lower measures of parasitism (Grant and Samways 2007). The Arrenurus sp. in this study seems to be restricted to bog habitats; this species is absent from $N$. irene, Enallagma ebrium and Ischnura verticalis in surrounding marsh habitats (data not shown), while it was on these host species at bog sites. The water mites sampled from surrounding habitats do not have the same shapes and textures of the sclerotized characters as this Arrenurus sp. (i.e. the dorsal shield sclerite is a different size, legs are different lengths and their excretory pores are shaped differently).

I am proposing that as a habitat-specialist, one host species has the upper hand in the co-evolutionary arms race with a generalist parasite, because the habitat specialist is exposed to fewer parasite species. This habitat specialist has evolved specific recognition of local parasites, and can maintain resistance because it is in a closed system where all host individuals have the potential to become infested by that parasite, thereby selecting for resistance. On the other hand, the more regionally well-represented host species has to cope with many different parasite species such that species specific resistance to any particular parasite might be too costly to maintain. Related to this point, the parasite has evolved to bypass the general resistance presented by the widespread host. This hypothesis of bog origin of the mite followed by bog invasion of alternative host species leading to strong sibling host species differences in resistance could be further tested with analysis of candidate genes responsible for parasite recognition and inferences about their molecular evolution. 


\section{CHAPTER SEVEN}

\section{General Discussion}




\section{General Discussion}

A central goal of evolutionary ecology is to understand factors influencing the numbers and relative strengths of species interactions. Species interactions are especially tractable for host-parasite associations because of the often obligate association parasites have with one or more host species. Linkages between host geographic distribution characteristics and measures of parasitism have been documented in mammals (Morand and Krasnov 2010), birds (Tella et al. 1999) and bees (Gillespie 2010). Such studies are useful in evaluating whether host ecological attributes influence species associations. In this thesis, I was specifically interested in determining which intrinsic and extrinsic host factors were predictive of levels of susceptibility and resistance to parasitism. My research addressed key gaps in explaining the reasons for particular host-parasites associations among invertebrates (insect hosts and acarine or protozoan parasites) by evaluating host species characteristics measured at multiple scales. My research is fundamental in its scope in attempting to understand the linkages between hosts and their parasites at multiple geographic and phylogenetic scales. Furthermore, it expands on how evolved recognition of parasitism might influence the evolution of particular host-parasite associations. Below, I highlight the key findings of each chapter, placing them into the context of novelty where applicable, and I offer future research directions.

Many previous studies on host-parasite associations focus their work from the viewpoint of the parasite and its impacts on host individuals (Poulin 2007). This emphasis stems in part from the fact that parasites tend to have a shorter life cycle than their hosts and it is believed that parasites have the upper hand in the co-evolutionary 
arms race (Price 1980). Additionally, many of the studies are tractable in that they focus on understanding the contemporary interactions between one host species and one parasite species, often at one or a few sites. As mentioned earlier, single host-single parasite species associations are rare in nature (Poulin 2007). Initially, I set out to test whether the results of studies on one host-one parasite species associations were scalable to investigations involving multiple hosts and multiple parasite species, which are the more common occurrence in nature. Indeed, I found that different host species, even if they are closely related phylogenetically, respond differently to the same or similar parasites. My research challenges past research in demonstrating that it is imperative to study hosts at broad taxonomic and ecological scales to have a better understanding of the evolution of species interactions. My research provides understanding of the interspecific variation in susceptibility and resistance to parasitism and is somewhat unique because of its scales of investigation. More specifically, this research examines variation of parasitism on a higher taxonomic and spatial scale than has been done before in insects.

First, my research challenges the Host Range hypothesis when there are restrictions of the taxonomic scope of the hosts. In Chapters 2 and 3, I determined if host geographic range size was a good predictor in explaining levels of parasitism in several pairs of host species grouped into pairs because of phylogenetic relatedness. I found that considering prevalence and intensity of parasitism, the associations varied depending on the group of parasite studied and on the host species pair studied. I observed that host geographic range is a good predictor for levels of gregarine parasitism (Chapter 2, Mlynarek et al. 2012) but less so for water mites (Chapter 3, Mlynarek et al. 2013). 
Chapter 2 was based on 14 associations between parasites believed to be generalists (gregarines), and sibling species pairs of their damselfly hosts (Argia, Calopterx, Enallagma Enallagma, Enallagma Chromatallagma, Ischnura, Lestes, Nehalennia). I found that in several species pairs, one species often had a higher measure(s) of parasitism; however, this was most often the less widely distributed host species of a species pair (Mlynarek et al. 2012). This pattern certainly occurred for Calopteryx, Enallagma Chromatallagma, and Ischnura species. There was no difference in levels of parasitism in the Argia species pair and in the Nehalennia species pair where the more widespread species had higher levels of gregarine parasitism.

In Chapter 3, I followed the same line of reasoning introduced in Chapter 2 but applied it to a different parasite group, Arrenurus water mites. I did this to determine whether the identity and life history of the parasite group would affect the observations of the relationship between host geographic range size and measures of parasitism. Relative geographic range size did predict relative prevalence of parasitism species pairs, but not reliably across all species pairs. To reiterate, there were significant differences in prevalence for Enallagma (Chromatallagma) species pair where the less widespread species had a higher prevalence of Arrenurus spp. However, in one species pair, Ischnura, the more widespread host species, I. verticalis, had significantly higher intensity of Arrenurus than the more geographically restricted host species I. posita. In these host-parasite associations, host geographic range was not a good predictor of parasitism. Across all other species pairs there were differences in geographic range but not in prevalence of ectoparasites. Furthermore, only in the Enallagma (Enallagma) 
species pair did the less widespread species have a higher mean intensity of infection by Arrenurus. Across all other species pairs, there were no species differences in mean intensity of mite parasitism.

Additionally, in Chapter 3, I used molecular barcoding (Cytochrome Oxidase I (COI) barcode) to identify the parasites to Operational Taxonomic Units (OTUs) to determine whether added taxonomic resolution of parasites would highlight better the observed relationship between host geographic range and levels of parasitism. There were nine OTUs within Arrenurus infecting the ten damselfly species. Three OTUs (\# 2, 7 and 9) appear to be specialized on a specific damselfly host species (E. signatum, A. moesta and A. violaceae, respectively). OTU 1 was a generalist infecting four host species collected in lakes. The remaining five OTUs (\# 3,4,5,6 and 8) infected at least two damselfly species each. Chapter 3 showed that water mites appear affiliated more with a site or habitat rather than on phylogenetically related host species. Except for OTU 1, collected from an I. verticalis host, all the water mites are restricted to one particular habitat type but were generally found on multiple host species.

Based on the results of the previous chapters (Chapter 2 and 3), host geographic range size did not seem to be a good predictor of levels of parasitism in closely related hosts. I therefore focused on exploring other host range characteristics that can be measured at different scales (Chapter 4). For Chapter 4, hosts were not paired with their closest evolutionary relative, but I did control for phylogeny using comparative methods. I also used molecular barcoding (COI) to identify OTUs as an additional factor. My 
question was whether host distribution characteristics were good predictor variables of the prevalence, intensity and number of parasite OTUs a host species' harboured. As with the results of Chapter 2 and based on Bayesian inference of water mites, water mites are quite generalist parasites and most host species were parasitized by more than one parasite species. The only species infected by one OTU was C resolutum. Enallagma ebrium was infected by seven OTUs. The rest of coenagrionids harbored between 2 and 6 OTUs. The main finding of Chapter 4 demonstrates that when controlling for phylogeny, host phenology and geographic range were the best predictors of water mite prevalence and intensity across the Coenagrionidae. Host species that were most active at the peak of the flight season and those with the largest geographic ranges were under the highest parasite pressure. The third measure of parasitism observed for this study, parasite range in host species, was predicted by host regional representation.

From observations made while analysing Chapters 3 and 4, I detected that certain host species showed resistance to their parasites. I observed darkened, un-engorged, dead water mites on the bodies of certain host individuals. Resistance has been documented before in a few studies focusing on single host species (Forbes et al. 1999). As variation in resistance is the flip side of variation in susceptibility, I wanted to explore whether a host's geographical range or immunity were potential reasons for this observed resistance across multiple species. Chapter 5 of my thesis is focused on the potential reasons for resistance variation including measures of host innate immunity and geographic range. The results show no clear relationship between PO activity and resistance across evolutionarily related host species which were grouped into species pairs and were 
parasitized by similar water mite parasites species. When comparing two closely related species, the species with higher prevalence of resisted mites was not the one with higher PO activity. The most extreme case was with Nehalennia; Nehalennia gracilis is the species that completely resists the parasite but there is no significant difference in PO activity. Within Argia and Ischnura species pairs, one species had significantly higher PO activity but resistance levels were not different between the two species within each species pair. Also of note was the fact that in Enallagma $C$ and Nehalennia, the less widespread species demonstrated highest prevalence of resistance.

There was an extreme case of interspecies differences in resistance and PO activity (Nehalennia) that warranted further investigation, which became the basis of Chapter 6. The Nehalennia species pair was unique in that two species N. gracilis, a host that resisted all water mites, and $N$. irene, a host that resisted none of the parasites but had higher PO activity, have very different ranges but morphologically are very similar. The two host species that I studied differ in their range, regional representation, and habitat specialization. Nehalennia irene has a widespread range $\left(4.03 \times 10^{6} \mathrm{Km}^{2}\right)$, is ubiquitous in freshwater habitats, is more regionally represented, and usually more locally abundant; whereas $N$. gracilis has a narrower range $\left(1.51 \times 10^{6} \mathrm{Km}^{2}\right)$, is a bog specialist, is restricted in its regional representation, usually less abundant (Walker 1953, Van Gossum et al. 2007, Mlynarek et al 2014) and has higher population genetic structure that $N$. irene (Iserbyt et al. 2010). Based on the differences between the two species and the fact that it was one Arrenurus species (based on molecular and morphological identification) infecting both hosts, I proposed that parasite recognition plays a major role. Nehalennia 
gracilis is thought to have evolved recognition because it encounters fewer parasites because it is so habitat restricted. There was the added finding that this type of resistance to Arrenurus sp. by $N$. gracilis (i.e. expected melanisation of the glands responsible for stylostome formation) has never been documented before.

In summary, I noted three important patterns from my research. First, I found that when considering evolutionary relationships between species within a family, prevalence and intensity of parasitism was explained by host phenology and geographic range size. Second, regional representation is an important factor in explaining the number of parasites species (OTUs as a proxy) a host can harbour. Third, constitutive measures of innate immunity are not good predictors of resistance and they do not relate to host distribution characteristics, but the genetic structure between the populations may be a key in understanding the evolution of resistance via parasite recognition.

\section{Future Directions}

The primary intention of this thesis was to determine the role of host characteristics on their associations with parasites. I tested whether host characteristics measured at different scales can predict levels and species diversity of parasitism. Habitat and parasite characteristics can also play major roles in host-parasite associations (Krasnov et al. 2004, Poulin 2007). Host, parasite and habitat characteristics are closely linked and changes in the characteristics of one of the three elements may influence the direction of evolution in the other two. 
Investigation of habitat characteristics

Habitat characteristics have not received as much attention as parasite and host characteristics but they can impact both host and parasite success at a site without even considering the species associations. Habitat characteristics may influence species success or abundances and they may influence the ability of a species to disperse. The habitat and the surrounding landscape can influence host-parasite associations. The importance of habitat specialization leading to genetic substructuring of populations leading to evolution of parasite recognition is hinted at toward the end of my thesis.

Investigating gregarine parasite characteristics

Ecological questions concerning damselfly-gregarine associations have yet to be fully addressed. Such studies would help inform us as to the generality of patterns derived in this body of work. There are many barriers to study the association with gregarines. First and foremost, the identity of gregarine species is difficult to ascertain. Appropriate molecular markers (18S rRNA) are being shown to be the best markers for species identification (Rueckert et al. 2011). Additionally, there is conflicting evidence concerning the impact of gregarines. Even the method of ingestion is not well understood. Therefore for the damselfly gregarine system, I would suggest studies on the processes of host infection by internal parasites. Additionally, there is also little knowledge of whether gregarines are able to infect nymphs and whether they are able to remain in the host through host eclosion. Through my preliminary observations, I determined that one damselfly nymph out of 100 was infected by one gregarine. Again 
manipulative, mesocosm experiments would help have an understanding of how infection occurs in damselfly-gregarine associations. Once there is a better understanding of the basic natural history of this association, tests of hypotheses will be possible.

\section{Investigating Arrenurus water mite characteristics}

Much research has been done to discern the importance of the role of parasite characteristics in host-parasite associations (Poulin 2007). However in the damselflywater mite system, more manipulative experimental studies that can further the understanding of this system. In the damselfly-water mite system, it is unclear how water mites chose their future hosts. They follow their chosen host paratenically but there are no studies that demonstrate whether mites recognize and can discriminate between hosts in the water column. Mesocosm experiments varying host species assemblages would enable the understanding of parasite host choice at the initial stages of infection and would allow us to make key observations concerning mite and damselfly behaviour in a controlled environment. Grooming has been well demonstrated in damselflies (Leung et al. 1999) but increasing species diversity may demonstrate how and whether hosts react to potential parasites. Developing an experimental study could demonstrate potential host associations by controlling for ecological constraints, which may be masked in natural settings (Poulin and Keeney 2007,Schmid-Hempel 2011). Additional projects on host and parasite recognition are going to be introduced and explored in the resistance section of this general discussion. 
Investigating host ranges in the damselflies-water mite system

A parasites host range represents a parasite characteristic that is one of the most unique and possibly flexible characteristics of that parasite species. The mechanisms determining why certain species are specialists and other generalists are not fully understood (Poulin 2007; Schmid-Hempel 2011). A parasite's ecological and physiological factors controlled by the evolutionary and biogeographical history of associated species determine the breadth of a species host range (Poulin 2007). It must be noted that the study of host ranges is based on the definition given to species boundaries of the group under study (Bickford et al. 2007; Poulin 2007). Currently, in additional to traditional morphological techniques, modern molecular techniques are becoming the most effective approaches for assessing species boundaries that will facilitate delimiting host ranges (Bickford et al. 2007).

Observations of natural occurring associations are an excellent method to understanding host ranges, but controlled experiments could give additional information about evolution and nature of the associations. There have been numerous live-choice experiments of Poecilochirus mites associated with Nicrophorus beetles, which helped the understanding of that host-symbiont relationship (Brown and Wilson 1992), but there have yet to be host-choice experiments on Arrenurus associated with odonates. In addition to observations of naturally occurring associations I would suggest doing livechoice experiments. These could provide us with a better understanding of host preferences and potentially the evolutionary history of damselfly-water mite associations. 


\section{Further exploration of resistance}

In my thesis work, I have observed instances of resistance and tested whether they can be explained by constitutive measures of innate immunity or host geographic characteristics (Chapters 5 and 6). The results suggest that hosts with closed genetically distinct populations may develop resistance more readily than their evolutionary close relatives that are in open populations and with increased genetic mixing (Chapter 6).

Arguments as to why species should differ in resistance expression can be found in Forbes and Mlynarek (2014). They suggest that future studies should flag associations and study instances where there are strong differences in resistance between host species to single species of parasites because such information might help researchers predict factors important to changes in host species range of parasite species over ecological and evolutionary time scales (Forbes and Mlynarek 2014). Like in the pattern observed with Nehalennia in Chapter 6, complete or near complete resistance has been observed in two instances of multi-host ectoparasitic mite subjected to two pairs of species of dragonflies (Sympetrum obtrusum and Sympetrum internum infected by Arrenurus planus (Forbes et al 1999) and Nanothemis bella and Leuchorrhinia intacta infected by Arrenurus sp. in (Lajeunesse et al. 2004)). In each comparison, the less abundant host species (S. internum and $N$. bella) showed absolute or near absolute resistance to the mite species whereas the common species was susceptible. One suggestion for this recurring pattern is that the common species is a larger target of selection for the parasite than is the less common species ( $c f$. Forbes and Robb 2008). This 'target' hypothesis makes sense as parasites could adapt to common host species by evading immune system recognition and would 
likely trade off with being recognized by the less common species (Forbes and Mlynarek 2014).

Intriguingly, in the case of Nehalennia, the more resistant species $N$. gracilis actually does not show more innate immunity than $N$. irene (Chapter 5) suggesting that the differences in resistance are probably aligned with differences in recognition and not host immune ability. It is also relevant to know the general life history of the species. In Nehalennia, $N$. gracilis is less regionally widespread compared with $N$. irene, generally less common at sites (Van Gossum et al. 2007) but genetically more structured between populations (Iserbyt et al. 2012) than $N$. irene. I would suggest comparing the life history differences and genetic structuring of $S$. obtrusum versus $S$. internum populations and even L. frigida versus $N$. bella to see which characteristics is a factor in resistance to their specific parasites. I would expect that the latter species in each comparison would exist in highly fragmented populations with limited gene flow. Based on the results from Chapter 5, the Enallagma Chromatallagma species pair (E. signatum and E. vesperum) would be worth investigating for this type of study. Enallagma vesperum, the more restricted species shows higher levels of resistance, just like the other three cases discussed above.

As further noted in Forbes and Mlynarek (2014), more research is needed on the factors accounting for differential resistance to parasites among related host species (or differential parasite-induced mortality). Resistance is probably common in nature because of immunity, but can be easily overlooked because the immune systems of the resistant species might remove all signs of past infections. Experimental infections would address 
whether differential resistance is real between host species. Following the experiments, researchers could examine the life history differences between species and look at the population genetics of related host species to determine if the partially or wholly resistant species are in more genetically sub-structured populations than the susceptible species. Finally, studies could be supplemented with molecular genetics work to determine if adaptive genetic variation for parasite recognition resides in either or both of the host species.

This thesis has allowed for a better understanding of damselfly-parasite associations especially in the damselfly-water mite system. It has also demonstrated how complex these associations really are. From this thesis, I now know the role host characteristics, especially host phenology, geographic range and regional representation, play in these systems. I propose that future studies focus on determining host ranges and further the knowledge of the evolution of resistance. 


\section{Literature Cited}

Abbott, J. C. 2007. OdonataCentral: An online resource for the distribution and identification of odonata. - Texas National Science Center, University of Texas at Austin. http://www.odonatacentral.org.

Abro, A. 1974. Gregarine Infection in Different Species of Odonata from Same Habitat. Zool Scr 3: 111-120.

Abro, A. 1971. Gregarines: their effects on damselflies (Odonata: Zygoptera). Entomologica Scandinavica 2: 294-300.

Altizer, S., Dobson, A., Hosseini, P., Hudson, P., Pascual, M. and Rohani, P. 2006. Seasonality and the dynamics of infectious diseases. - Ecol Lett 9: 467-484.

Armitage, S. and Siva-Jothy, M. 2005. Immune function responds to selection for cuticular colour in Tenebrio molitor. - Heredity 94: 650-656.

Arneberg, P., Skorping, A., Grenfell, B. and Read, A. 1998. Host densities as determinants of abundance in parasite communities. - Proceedings of the Royal Society B-Biological Sciences 265: 1283-1289.

Bickford, D., Lohman, D. J., Sodhi, N. S., Ng, P. K. L., Meier, R., Winker, K., Ingram, K. K. and Das, I. 2007. Cryptic species as a window on diversity and conservation. - Trends in Ecology \& Evolution 22: 148-155. 
Bidla, G., Hauling, T., Dushay, M. S. and Theopold, U. 2009. Activation of insect phenoloxidase after Injury: endogenous versus foreign elicitors. - Journal of Innate Immunity 1: 301-308.

Blomberg, S., Garland, T. and Ives, A. 2003. Testing for phylogenetic signal in comparative data: Behavioral traits are more labile. - Evolution 57: 717-745.

Bordes, F. and Morand, S. 2009. Parasite diversity: an overlooked metric of parasite pressures? - Oikos 118: 801-806.

Bordes, F., Morand, S., Kelt, D. A. and Van Vuren, D. H. 2009. Home Range and Parasite Diversity in Mammals. - Am Nat 173: 467-474.

Bried, J. T., Hager, B. J., Hunt, P. D., Fox, J. N., Jensen, H. J. and Vowels, K. M. 2012. Bias of reduced-effort community surveys for adult Odonata of lentic waters. Insect Conservation and Diversity 5: 213-222.

Brown, J. M. and Wilson, D. S. 1992. Local Specialization of Phoretic Mites on Sympatric Carrion Beetle Hosts. - Ecology 73: 463-478.

Brown, J. 1984. On the Relationship between Abundance and Distribution of Species. Am Nat 124: 255-279.

Brown, J., McPeek, M. and May, M. 2000. A phylogenetic perspective on habitat shifts and diversity in the north American Enallagma damselflies. - Syst Biol 49: 697712. 
Burnham, K. P., Anderson, D. R. and Burnham, K. P. 2002. Model selection and multimodel inference a practical information-theoretic approach. - Springer.

Bush, A. O. 2001. Parasitism : the diversity and ecology of animal parasites. - Cambridge University Press.

Campbell, K. U., Klompen, H. and Crist, T. O. 2013. The diversity and host specificity of mites associated with ants: the roles of ecological and life history traits of ant hosts. - Insectes Soc 60: 31-41.

Canales-Lazcano, J., Contreras-Garduno, J. and Cordoba-Aguilar, A. 2005. Fitnessrelated attributes and gregarine burden in a nonterritorial damselfly Enallagma praevarum Hagen (Zygoptera: Coenagrionidae). - Odonatologica 34: 123-130.

Carle, F., Kjer, K. and May, M. 2008. Evolution of Odonata, with special reference to Coenagrionoidea (Zygoptera) - Arthropod Systematics and Phylogeny 66: 37-44.

Chippindale, P., Dave, V., Whitmore, D. and Robinson, J. 1999. Phylogenetic relationships of North American damselflies of the genus Ischnura (Odonata : Zygoptera : Coenagrionidae) based on sequences of three mitochondrial genes. Mol Phylogenet Evol 11: 110-121.

Cielocha, J. J., Cook, T. J. and Clopton, R. E. 2011. Host utilization and distribution of Nubenocephalid gregarines (Eugregarinorida: Actinocephalidae) parasitizing Argia 
spp. (Odonata: Zygoptera) in the Central United States. - Comparative Parasitology 78: $152-160$.

Clopton, R. and Gold, R. 1996. Host specificity of Gregarina blattarum von Siebold, 1839 (Apicomplexa: Eugregarinida) among five species of domiciliary cockroaches. - J Invertebr Pathol 67: 219-223.

Clopton, R. E. 2009. Phylogenetic Relationships, Evolution, and Systematic Revision of the Septate Gregarines (Apicomplexa: Eugregarinorida: Septatorina). Comparative Parasitology 76: 167-190.

Cognato, A. I. 2006. Standard percent DNA sequence difference for insects does not predict species boundaries. - J Econ Entomol 99: 1037-1045.

Corbet, P. S. 1999. Dragonflies : behavior and ecology of Odonata. - Comstock Pub. Associates.

Corby-Harris, V. and Promislow, D. E. L. 2008. Host ecology shapes geographical variation for resistance to bacterial infection in Drosophila melanogaster. - J Anim Ecol 77: 768-776.

Cordoba-Aguilar, A., Salamanca-Ocana, J. and Lopezaraiza, M. 2003. Female reproductive decisions and parasite burden in a calopterygid damselfly (Insecta : Odonata). - Anim Behav 66: 81-87. 
Cotter, S., Kruuk, L. and Wilson, K. 2004. Costs of resistance: genetic correlations and potential trade-offs in an insect immune system. - J Evol Biol 17: 421-429.

Crozier, R. H., Dunnett, L. J. and Agapow, P. 2005. Phylogenetic biodiversity assessment based on systematic nomenclature. - Evolutionary Bioinformatics 1: 11-36.

Davies, N., Bourke, A. and Brooke, M. 1989. Cuckoos and parasitic ants - interspecific brood parasitism as an evolutionary arms-race. - Trends in Ecology \& Evolution 4: 274-278.

Dawkins, R. and Krebs, J. R. 1979. Arms races between and within species. Proceedings of the Royal Society Series B-Biological Sciences 205: 489-511.

Detwiler, J. and Janovy, J., Jr. 2008. The role of phylogeny and ecology in experimental host specificity: Insights from a eugregarine-host system. - J Parasitol 94: 7-12.

Dritschilo, W., Cornell, H., Nafus, D. and O'Connor, B. 1975. Insular Biogeography Mice and Mites. - Science 190: 467-469.

Dubovskiy, I. M., Whitten, M. M. A., Kryukov, V. Y., Yaroslavtseva, O. N., Grizanova, E. V., Greig, C., Mukherjee, K., Vilcinskas, A., Mitkovets, P. V., Glupov, V. V. and Butt, T. M. 2013. More than a colour change: insect melanism, disease resistance and fecundity. - Proceedings of the Royal Society B-Biological Sciences 280. 
Dumont, H. J., Vierstraete, A. and Vanfleteren, J. R. 2010. A molecular phylogeny of the Odonata (Insecta). - Syst Entomol 35: 6-18.

Durrer, S. and Schmid-Hempel, P. 1995. Parasites and the regional distribution of bumblebee species. - Ecography 18: 114-122.

Foitzik, S., DeHeer, C., Hunjan, D. and Herbers, J. 2001. Coevolution in host-parasite systems: behavioural strategies of slave-making ants and their hosts. - Proceedings of the Royal Society B-Biological Sciences 268: 1139-1146.

Forbes, M. R. and Mlynarek, J. J. 2014. A hypothesis to explain host species differences in resistance to multi-host parasites. - Ideas in Ecology and Evolution 7: 17-24.

Forbes, M. R. and Robb, T. 2008. Testing hypotheses about parasite-mediated selection using odonate hosts. - Dragonflies and Damselflies: Model Organisms for Ecological and Evolutionary Research : 175-188.

Forbes, M. R. and Baker, R. 1991. Condition and fecundity of the damselfly, EnallagmaEbrium (Hagen) - the importance of ectoparasites. - Oecologia 86: 335-341.

Forbes, M. R., Mlynarek, J. J., Allison, J. and Hecker, K. R. 2012. Seasonality of gregarine parasitism in the damselfly, Nehalennia irene: understanding unimodal patterns. - Parasitol Res 110: 245-250. 
Forbes, M., Muma, K. and Smith, B. 1999. Parasitism of Sympetrum dragonflies by Arrenurus planus mites: maintenance of resistance particular to one species. - Int J Parasitol 29: 991-999.

Forsyth, A. and Montgomerie, R. 1987. Alternative reproductive tactics in the territorial damselfly Calopteryx-maculata - sneaking by older males. - Behav Ecol Sociobiol 21: 73-81.

Gaston, K. 1990. Patterns in the geographical ranges of species. - Biol Rev Camb Philos Soc 65: 105-129.

Gibson, J. F., Kelso, S. and Skeyington, J. H. 2010. Band-cutting no more: A method for the isolation and purification of target PCR bands from multiplex PCR products using new technology. - Mol Phylogenet Evol 56: 1126-1128.

Gillespie, S. 2010. Factors affecting parasite prevalence among wild bumblebees. - Ecol Entomol 35: 737-747.

Goloboff, P. A., Farris, J. S. and Nixon, K. C. 2008. TNT, a free program for phylogenetic analysis. - Cladistics 24: 774-786.

Gonzalez-Santoyo, I., Cordoba-Aguilar, A., Gonzalez-Tokman, D. M. and LanzMendoza, H. 2010. Phenoloxidase activity and melanization do not always covary with sexual trait expression in Hetaerina damselflies (Insecta: Calopterygidae). Behaviour 147: 1285-1307. 
Gonzalez-Santoyo, I. and Cordoba-Aguilar, A. 2012. Phenoloxidase: a key component of the insect immune system. - Entomol Exp Appl 142: 1-16.

Grant, P. B. C. and Samways, M. J. 2007. Ectoparasitic mites infest common and widespread but not rare and red-listed dragonfly species - Odonatologica 36: 255262.

Gregory, R. 1990. Parasites and host geographic range as illustrated by waterfowl. Funct Ecol 4: 645-654.

Guegan, J. F., Lambert, A., Leveque, C., Combes, C. and Euzet, L. 1992. Can host body size explain the parasite species richness in tropical fresh-water fishes. - Oecologia 90: 197-204.

Ham, P. J., Hagen, H., Baxter, A. J. and Grunewald, J. 1995. Mechanisms of resistance to Onchocerca infection in blackflies. - Parasitology Today 11: 63-67.

Hanski, I. 1982. On patterns of temporal and spatial variation in animal populations. Ann Zool Fenn 19: 21-37.

Hassall, C. 2012. Predicting the distributions of under-recorded Odonata using species distribution models. - Insect Conservation and Diversity 5: 192-201.

Hassall, C., Thompson, D. J., French, G. C. and Harvey, I. F. 2007. Historical changes in the phenology of British Odonata are related to climate. - Global Change Biol 13: 933-941. 
Hassall, C., Lowe, C. D., Harvey, I. F., Watts, P. C. and Thompson, D. J. 2010.

Phenology determines seasonal variation in ectoparasite loads in a natural insect population. - Ecol Entomol 35: 514-522.

Hecker, K., Forbes, M. and Leonard, N. 2002. Parasitism of damselflies (Enallagma boreale) by gregarines: sex biases and relations to adult survivorship. - Canadian Journal of Zoology-Revue Canadienne De Zoologie 80: 162-168.

Hefferman, J., Soranno, P., Angilletta Jr, M., Buckley, L., Gruner, D., Keitt, T., Kellner, J., Kominoski, J., Rocha, A., Xiao, J., Harms, T., Goring, S., Koenig, L., McDowell, W., Powell, H., Richardson, A., Stow, C., Vargas, R. and Weathers, K. 2014. Macrosystems ecology: understanding ecological patterns and processes at continental scales. - Frontiers in Ecology and Evolution 12: 5-14.

Huelsenbeck, J. P. and Ronquist, F. 2001. MRBAYES: Bayesian inference of phylogenetic trees. - Bioinformatics 17: 754-755.

Ilvonen, S., Ilvonen, J. J., Kaunisto, K. M., Krams, I. and Suhonen, J. 2011. Can infection by eugregarine parasites mediate species coexistence in Calopteryx damselflies? Ecol Entomol 36: 582-587.

Iserbyt, A., Bots, J., Van Gossum, H. and Jordaens, K. 2010. Did historical events shape current geographic variation in morph frequencies of a polymorphic damselfly? - J Zool 282: 256-265. 
Iserbyt, A., Van Gossum, H. and Stoks, R. 2012. Biogeographical Survey Identifies Consistent Alternative Physiological Optima and a Minor Role for Environmental Drivers in Maintaining a Polymorphism. - Plos One 7: e32648.

Ivanova, N. V., Dewaard, J. R. and Hebert, P. D. N. 2006. An inexpensive, automationfriendly protocol for recovering high-quality DNA. - Molecular Ecology Notes 6: 998-1002.

Jaenike, J. and Perlman, S. J. 2002. Ecology and evolution of host-parasite associations: Mycophagous Drosophila and their parasitic nematodes. - Am Nat 160: S23-S39.

Kanost, M. R. and Gorman, M. J. 2008. Phenoloxidases in Insect Immunity. - In: Beckage, N. (ed.), Insect Immunology. Academic Press/Elsevier, pp. 69-96.

Kaunisto, K. M., Viitaniemi, H. M., Leder, E. H. and Suhonen, J. 2013. Association between host's genetic diversity and parasite burden in damselflies. - J Evol Biol 26: 1784-1789.

Kennedy, C. R. and Bush, A. O. 1994. The relationship between pattern and scale in parasite communities - a stranger in a strange land. - Parasitology 109: 187-196.

Klingenberg, C. P. 2011. MorphoJ: an integrated software package for geometric morphometrics. - Molecular Ecology Resources 11: 353-357.

Knee, W., Beaulieu, F., Skevington, J. H., Kelso, S. and Forbes, M. R. 2012. Cryptic species of mites (Uropodoidea: Uroobovella spp.) associated with burying beetles 
(Silphidae: Nicrophorus): The collapse of a host generalist revealed by molecular and morphological analyses. - Mol Phylogenet Evol 65: 276-286.

Krasnov, B., Khokhlova, I. and Shenbrot, G. 2002. The effect of host density on ectoparasite distribution: An example of a rodent parasitized by fleas. - Ecology 83: 164-175.

Krasnov, B. R., Shenbrot, G. I., Medvedev, S. G., Vatschenok, V. S. and Khokhlova, I. S. 1997. Host-habitat relations as an important determinant of spatial distribution of flea assemblages (Siphonaptera) on rodents in the Negev desert. - Parasitology 114: 159-173.

Krasnov, B. R., Poulin, R. and Mouillot, D. 2011. Scale-dependence of phylogenetic signal in ecological traits of ectoparasites. - Ecography 34: 114-122.

Krasnov, B., Stanko, M. and Morand, S. 2006. Age-dependent flea (Siphonaptera) parasitism in rodents: A host's life history matters. - J Parasitol 92: 242-248.

Krasnov, B., Shenbrot, G., Khokhlova, I. and Degen, A. 2004. Flea species richness and parameters of host body, host geography and host 'milieu'. - J Anim Ecol 73: 11211128.

Lajeunesse, M. and Forbes, M. 2002. Host range and local parasite adaptation. Proceedings of the Royal Society B-Biological Sciences 269: 703-710. 
Langmore, N., Hunt, S. and Kilner, R. 2003. Escalation of a coevolutionary arms race through host rejection of brood parasitic young. - Nature 422: 157-160.

Leung, B., Baker, R. and Forbes, M. 1999. Grooming decisions by damselflies, agespecific colonisation by water mites, and the probability of successful parasitism. Int J Parasitol 29: 397-402.

Levin, S. 1992. The problem of pattern and scale in ecology. - Ecology 73: 1943-1967.

Lindenfors, P., Nunn, C. L., Jones, K. E., Cunningham, A. A., Sechrest, W. and Gittleman, J. L. 2007. Parasite species richness in carnivores: effects of host body mass, latitude, geographical range and population density. - Global Ecol Biogeogr 16: 496-509.

Locklin, J. L. and Vodopich, D. S. 2010. Patterns of gregarine parasitism in dragonflies: host, habitat, and seasonality. - Parasitol Res 107: 75-87.

Lopez, J. E. 2005. Parasite prevalence and the size of host populations: An experimental test. - J Parasitol 91: 32-37.

Maddison, W. P. and Maddison, D. R. 2011. Mesquite: a modular system for evolutionary analysis. - 2.75. http://mesquiteproject.org.

Marmaras, V., Charalambidis, N. and Zervas, C. 1996. Immune response in insects: The role of phenoloxidase in defense reactions in relation to melanization and sclerotization. - Arch Insect Biochem Physiol 31: 119-133. 
Martin, P., Dabert, M. and Dabert, J. 2010. Molecular evidence for species separation in the water mite Hygrobates nigromaculatus Lebert, 1879 (Acari, Hydrachnidia): evolutionary consequences of the loss of larval parasitism. - Aquat Sci 72: 347-360.

May, M. 2002. Phylogeny and taxonomy of the damselfly genus Enallagma and related taxa (Odonata : Zygoptera : Coenagrionidae). - Syst Entomol 27: 387-408.

Miller, M., Pfeiffer, W. and Schwartz, T. 2010. Creating the CIPRES science gateway for inference of large phylogenetic trees . - In: Anonymous Proceedings of the Gateway Computing Environments Workshop (GCE). IEEE, pp. 1-8.

Mitchell, R. 1955. Anatomy, life history, and evolution of the mites parasitizing freshwater mussels. - Miscellaneous Publications of the Museum of Zoology, University of Michigan 89: 1-52.

Mlynarek, J. J., Knee, W. and Forbes, M. R. 2014. Explaining susceptibility and resistance to a multi-host parasite - Evolutionary Biology 41: 115-122.

Mlynarek, J. J., Knee, W. and Forbes, M. R. 2013. Relative geographic range of sibling species of host damselflies does not reliably predict differential parasitism by water mites. - BMC ecology 13: 50.

Mlynarek, J. J., Hassall, C. and Forbes, M. R. 2012. Higher gregarine parasitism often in sibling species of host damselflies with smaller geographical distributions. - Ecol Entomol 37: 419-425. 
Mlynarek, J. J., Bert, D. G., Haydee Peralta-Vazquez, G., James, J. A. and Forbes, M. R. 2011. Relationships between gregarine infection in damselflies, wetland type, and landscape characteristics. - Can Entomol 143: 460-469.

Morand, S., Bordes, F., Pisanu, B., de Bellocq, J. G. and Krasnov, B. 2010. The geography of defence. - In: Morand, S. and Krasnov, B. (eds.), <br />The Biogeography of Host-Parasite Interactions Oxford University Press, pp. 159-173.

Morand, S. and Krasnov, B. R. 2010. The biogeography of host-parasite interactions. Oxford University Press.

Moreno-Garcia, M., Cordoba-Aguilar, A., Conde, R. and Lanz-Mendoza, H. 2013. Current immunity markers in insect ecological immunology: assumed trade-offs and methodological issues. - Bull Entomol Res 103: 127-139.

Munoz, G., Grutter, A. S. and Cribb, T. H. 2007. Structure of the parasite communities of a coral reef fish assemblage (Labridae): Testing ecological and phylogenetic host factors. - J Parasitol 93: 17-30.

Nagel, L., Mlynarek, J. J. and Forbes, M. R. 2011. Immune response to nylon filaments in two damselfly species that differ in their resistance to ectoparasitic mites. - Ecol Entomol 36: 736-743.

Nylander, J. A. A. 2004. MrModeltest v2. - Program distributed by the author, Uppsala Evolutionary Biology Center, Uppsala University. 
O'Grady, E. and May, M. 2003. A phylogenetic reassessment of the subfamilies of Coenagrionidae (Odonata : Zygoptera). - J Nat Hist 37: 2807-2834.

Oksanen, J., Blanchet, F. G., Kindt, R., Legendre, P., Minchin, P. R., O'Hara, R. B., Simpson, G. L., Solymos, P., Stevens, M. H. M. and Wagner, H. 2013. vegan: community ecology package. - R package v.2.0-7.

Orme, D., Freckleton, R., Thomas, G., Petzoldt, T., Fritz, S., Isaac, N. and Pearse, W. 2012. caper: Comparative Analyses of Phylogenetics and Evolution in R. - R package version 0.5 .

Paivinen, J., Grapputo, A., Kaitala, V., Komonen, A., Kotiaho, J., Saarinen, K. and Wahlberg, N. 2005. Negative density-distribution relationship in butterflies. - BMC Biology 3: 5.

Patterson, B. D., Dick, C. W. and Dittmar, K. 2008. Parasitism by bat flies (Diptera : Streblidae) on neotropical bats: effects of host body size, distribution, and abundance. - Parasitol Res 103: 1091-1100.

Pauwels, K., De Meester, L., Decaestecker, E. and Stoks, R. 2011. Phenoloxidase but not lytic activity reflects resistance against Pasteuria ramosa in Daphnia magna. Biology Letters 7: 156-159. 
Perez-Orella, C. and Schulte-Hostedde, A. 2005. Effects of sex and body size on ectoparasite loads in the northern flying squirrel (Glaucomys sabrinus). - Canadian Journal of Zoology-Revue Canadienne De Zoologie 83: 1381-1385.

Pianka, E. R. 1983. Evolutionary ecology. - Harper \& Row.

Pielou, E. 1974. Biogeographic Range Comparisons and Evidence of Geographic Variation in Host-Parasite Relations. - Ecology 55: 1359-1367.

Poulin, R. 2007. Evolutionary ecology of parasites. - Princeton University Press.

Poulin, R. 2005. Relative infection levels and taxonomic distances among the host species used by a parasite: insights into parasite specialization. - Parasitology 130: 109-115.

Poulin, R. 1991. Group-Living and Infestation by Ectoparasites in Passerines. - Condor 93: 418-423.

Poulin, R. and Keeney, D. B. 2007. Host specificity under molecular and experimental scrutiny. - Trends in parasitology 24: 24-28.

Poulin, R. and George-Nascimento, M. 2007. The scaling of total parasite biomass with host body mass. - Int J Parasitol 37: 359-364.

Poulin, R. and Morand, S. 2004. Parasite biodiversity. - Smithsonian Books.

Price, P. W. 1980. Evolutionary biology of parasites. - Princeton University Press. 
Price, P. and Clancy, K. 1983. Patterns in Number of Helminth Parasite Species in FreshWater Fishes. - J Parasitol 69: 449-454.

Price, P., Westoby, M. and Rice, B. 1988. Parasite-Mediated Competition - some Predictions and Tests. - Am Nat 131: 544-555.

Ratnasingham, S. and Hebert, P. D. N. 2007. BOLD: The Barcode of Life Data System (www.barcodinglife.org). - Molecular Ecology Notes 7: 355-364.

Rohlf, F. J. 2009. tpsDig. Department of Ecology and Evolution. State University of New York - 2.14. http://life.bio.sunysb.edu.morph/.

Ronquist, F. and Huelsenbeck, J. P. 2003. MrBayes 3: Bayesian phylogenetic inference under mixed models. - Bioinformatics 19: 1572-1574.

Rozsa, L., Reiczigel, J. and Majoros, G. 2000. Quantifying parasites in samples of hosts. - J Parasitol 86: 228-232.

Rueckert, S., Villette, P. M. A. H. and Leander, B. S. 2011. Species Boundaries in Gregarine Apicomplexan Parasites: A Case Study-Comparison of Morphometric and Molecular Variability in Lecudina cf. tuzetae (Eugregarinorida, Lecudinidae). J Eukaryot Microbiol 58: 275-283.

Rynkiewicz, E. C., Hawlena, H., Durden, L. A., Hastriter, M. W., Demas, G. E. and Clay, K. 2013. Associations between innate immune function and ectoparasites in wild rodent hosts. - Parasitol Res 112: 1763-1770. 
Schmid-Hempel, P. 2005. Evolutionary ecology of insect immune defenses. - Annu Rev Entomol 50: 529-551.

Schmid-Hempel, P. 2011. Evolutionary parasitology : the integrated study of infections, immunology, ecology, and genetics. - Oxford University Press.

Schneider, C. A., Rasband, W. S. and Eliceiri, K. W. 2012. NIH Image to ImageJ: 25 years of image analysis. - Nature Methods 9: 671-675.

Schwarzenbach, G. A. and Ward, P. I. 2007. Phenoloxidase activity and pathogen resistance in yellow dung flies Scathophaga stercoraria. - J Evol Biol 20: 21922199.

Simková, A., Morand, S., Matejusova, I., Jurajda, P. V. and Gelnar, M. 2001. Local and regional influences on patterns of parasite species richness of central European fishes. - Biodivers Conserv 10: 511-525.

Smilanich, A. M., Dyer, L. A. and Gentry, G. L. 2009. The insect immune response and other putative defenses as effective predictors of parasitism. - Ecology 90: 14341440.

Smith, I., Cook, D. and Smith, B. 2010. Water mites (Hydrachnidiae) and other arachnids - In: Thorp, J. and Covich, A. (eds.), Ecology and Classification of North American Freshwater Invertebrates 3rd edition Academic Press, pp. 485-587. 
Soderhall, K. and Cerenius, L. 1998. Role of the prophenoloxidase-activating system in invertebrate immunity. - Curr Opin Immunol 10: 23-28.

Stoks, R., De Block, M., Slos, S., Van Doorslaer, W. and Rolff, J. 2006. Time constraints mediate predator-induced plasticity in immune function, condition, and life history. - Ecology 87: 809-815.

Sugumaran, M. 2002. Comparative biochemistry of eumelanogenesis and the protective roles of phenoloxidase and melanin in insects. - Pigment Cell Research 15: 2-9.

Sugumaran, M., Nellaiappan, K. and Valivittan, K. 2000. A new mechanism for the control of phenoloxidase activity: Inhibition and complex formation with quinone isomerase. - Arch Biochem Biophys 379: 252-260.

Swofford, D. L. 2003. PAUP*. Phylogenetic Analysis Using Parsimony (*and Other Methods). - 4.0b10. Sinauer Associates.

Tack, A. J. M., Horns, F. and Laine, A. 2014. The Impact of Spatial Scale and Habitat Configuration on Patterns of Trait Variation and Local Adaptation in a Wild Plant Parasite. - Evolution 68: 176-189.

Teder, T. and Tammaru, T. 2003. Short-term indirect interactions between two moth (Lepidoptera : Noctuidae) species mediated by shared parasitoids: The benefit of being scarce. - Eur J Entomol 100: 323-328. 
Tella, J., Blanco, G., Forero, M., Gajon, A., Donazar, J. and Hiraldo, F. 1999. Habitat, world geographic range, and embryonic development of hosts explain the prevalence of avian hematozoa at small spatial and phylogenetic scales. - Proc Natl Acad Sci USA 96: 1785-1789.

Thompson, J. N. 2005. The geographic mosaic of coevolution. - University of Chicago Press.

Tripet, F. and Richner, H. 1997. The coevolutionary potential of a 'generalist' parasite, the hen flea Ceratophyllus gallinae. - Parasitology 115: 419-427.

Van Gossum, H., Beirinckx, K., Forbes, M. R. and Sherratt, T. N. 2007. Do current hypotheses explain continental and seasonal variation in female morph frequencies of the damselfly, Nehalennia irene? - Biol J Linn Soc 90: 501-508.

Vaughn, C. C. and Taylor, C. M. 2000. Macroecology of a host-parasite relationship. Ecography 23: 11-20.

Vesakoski, O. and Jomalainen, V. 2013. Ignored patterns in studies of local adaptations: When the grass is greener on the allopatric site. - Ideas in Ecology and Evolution 6: 32-36.

Walker, E. M. 1953. The Odonata of Canada and Alaska. - University Press.

Webb, C. 2000. Exploring the phylogenetic structure of ecological communities: An example for rain forest trees. - Am Nat 156: 145-155. 
Whitehorn, P. R., Tinsley, M. C., Brown, M. J. F., Darvill, B. and Goulson, D. 2011.

Genetic diversity, parasite prevalence and immunity in wild bumblebees. Proceedings of the Royal Society B-Biological Sciences 278: 1195-1202.

Whitlock, M. C. 1996. The red queen beats the jack-of-all-trades: The limitations on the evolution of phenotypic plasticity and niche breadth. - Am Nat 148: S65-S77.

Wiens, J. 1989. Spatial Scaling in Ecology. - Funct Ecol 3: 385-397.

Wilson, K. and Cotter, S. C. 2013. Host-Parasite Interactions and the Evolution of Immune Defense. - In: Brockmann, H. J., Roper, T. J., Naguib, M. and Barrett, L. (eds.), Advances in the Study of Behavior. Elsevier Inc.: Academic Press, pp. 81174.

Yourth, C., Forbes, M. and Smith, B. 2002. Immune expression in a damselfly is related to time of season, not to fluctuating asymmetry or host size. - Ecol Entomol 27: 123-128.

Yourth, C., Forbes, M. and Smith, B. 2001. On understanding variation in immune expression of the damselflies Lestes spp. - Canadian Journal of Zoology-Revue Canadienne De Zoologie 79: 815-821.

Zar, J. H. 1999. Biostatistical analysis. - Prentice Hall. 
Zawal, A. 2008. Morphological characteristics of water mite larvae of the genus Arrenurus Duges, 1834, with notes on the phylogeny of the genus and an identification key. - Zootaxa : 1-75.

Zuk, M. 1987. Seasonal and Individual Variation in Gregarine Parasite Levels in the Field Crickets Gryllus-Veletis and Gryllus-Pennsylvanicus. - Ecol Entomol 12: 341-348. 
Appendix A. Operational definitions of terms bolded in the text.

\begin{tabular}{|c|c|}
\hline Term & Operational definition \\
\hline Coevolution & $\begin{array}{l}\text { Reciprocal evolutionary change } \\
\text { (Thompson 2005) }\end{array}$ \\
\hline Direct life cycle & $\begin{array}{l}\text { A parasite that only infect one host } \\
\text { throughout their life (Bush 2001). }\end{array}$ \\
\hline Generalist parasite & $\begin{array}{l}\text { Parasite that is able to infect more than one } \\
\text { host species (Poulin 2007) }\end{array}$ \\
\hline Geographic range & $\begin{array}{l}\text { The total area occupied by a species } \\
\text { (Gaston 1990) }\end{array}$ \\
\hline Gregarine & $\begin{array}{l}\text { Protozoan gut parasite of insects and other } \\
\text { invertebrates (Bush 2001). }\end{array}$ \\
\hline Host range & $\begin{array}{l}\text { Number of realized host species that a } \\
\text { given parasites species can exploit } \\
\text { (Schmid-Hempel 2010) }\end{array}$ \\
\hline Innate immunity & $\begin{array}{l}\text { The capacity to mount a general response } \\
\text { to a challenge such as parasites (Schmid- } \\
\text { Hempel 2010) }\end{array}$ \\
\hline Intensity & $\begin{array}{l}\text { Average number of parasite individuals } \\
\text { only considering infected host individuals } \\
\text { (Rozsa et al 2010) }\end{array}$ \\
\hline local abundance & $\begin{array}{l}\text { Density of a species of interest across a site } \\
\text { or across a series of sites (Gaston 1990). }\end{array}$ \\
\hline Measures of parasitism & $\begin{array}{l}\text { The number or diversity of parasites that an } \\
\text { individual host, a host population or a host } \\
\text { species carries (Schmid-Hempel 2010) }\end{array}$ \\
\hline Parasite Range & $\begin{array}{l}\text { Number of parasite species that have been } \\
\text { documented on a host species (Poulin } \\
\text { 2007) }\end{array}$ \\
\hline Phenology & $\begin{array}{l}\text { Seasonal variation in the life history of a } \\
\text { plan or animal (Lieth 1974). }\end{array}$ \\
\hline PO (Phenoloxidase) & $\begin{array}{l}\text { An important enzyme in invertebrate } \\
\text { immune defences (Schmid-Hempel 2010) }\end{array}$ \\
\hline Prevalence & $\begin{array}{l}\text { Proportion in a host population that is } \\
\text { infected with at least one parasite } \\
\text { individual (Bush et al 1997) }\end{array}$ \\
\hline
\end{tabular}


Appendix

Appendix A. Continued

\begin{tabular}{|l|l|}
\hline Regional representation & $\begin{array}{l}\text { The proportion of sites in which a species } \\
\text { occurs (Magurran and Henderson 2011). }\end{array}$ \\
\hline Resistance & $\begin{array}{l}\text { The ability of a host to limit parasite } \\
\text { burden (Raberg et al 2009) }\end{array}$ \\
\hline Specialist parasite & $\begin{array}{l}\text { Parasite that is limited to be able to infect } \\
\text { one host species successfully (Rigaud et al. } \\
\text { 2010) }\end{array}$ \\
\hline Species richness & $\begin{array}{l}\text { Number of different species represented in } \\
\text { an ecological community. It is usually } \\
\text { dependent on the scale and region of study }\end{array}$ \\
\hline Susceptibility & $\begin{array}{l}\text { Failure to resist an infection (Schmid- } \\
\text { Hempel 2010) }\end{array}$ \\
\hline Water mite & $\begin{array}{l}\text { A group of Acari mites in the group } \\
\text { Hydrachnidae that have an aquatic life } \\
\text { style. Many of which are external parasites } \\
\text { of arthropods (Smith et al 2010). }\end{array}$ \\
\hline
\end{tabular}

